\title{
Pharmacogenetics of tenofovir and emtricitabine penetration into cerebrospinal fluid
}

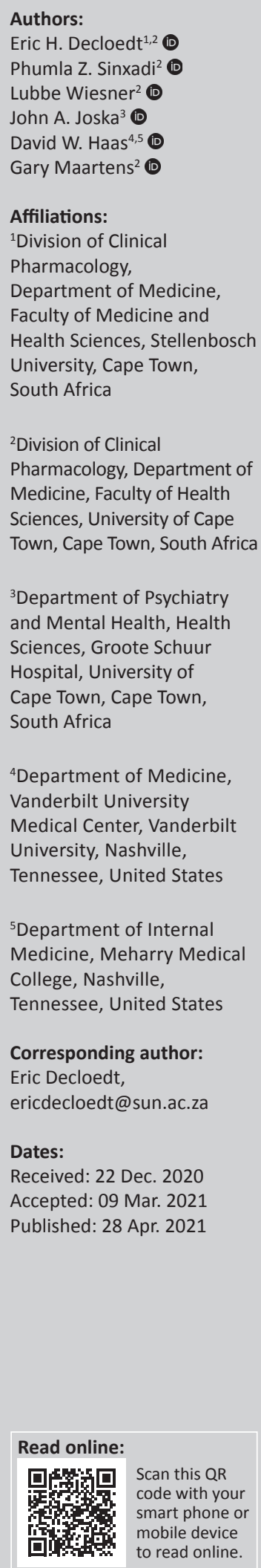

Background: Blood-cerebrospinal fluid (CSF) barrier transporters affect the influx and efflux of drugs. The antiretrovirals tenofovir and emtricitabine may be substrates of blood-brain barrier (BBB) and blood-CSF barrier transporters, but data are limited regarding the pharmacogenetics and pharmacokinetics of their central nervous system (CNS) penetration.

Objectives: We investigated genetic polymorphisms associated with CSF disposition of tenofovir and emtricitabine.

Method: We collected paired plasma and CSF samples from 47 HIV-positive black South African adults who were virologically suppressed on efavirenz, tenofovir and emtricitabine. We considered 1846 single-nucleotide polymorphisms from seven relevant transporter genes (ABCC5, ABCG2, ABCB1, SLCO2B1, SCLO1A2, SLCO1B1 and ABCC4) and 782 met a linkage disequilibrium (LD)-pruning threshold.

Results: The geometric mean (95\% confidence interval [CI]) values for tenofovir and emtricitabine CSF-to-plasma concentration ratios were $0.023(0.021-0.026)$ and 0.528 (0.460-0.605), respectively. In linear regression models, the lowest $p$-value for association with the tenofovir CSF-to-plasma ratio was ABCB1 rs1989830 $\left(p=1.2 \times 10^{-3}\right)$ and for emtricitabine, it was $A B C C 5$ rs11921035 $\left(p=1.4 \times 10^{-3}\right)$. None withstood correction for multiple testing.

Conclusion: No genetic polymorphisms were associated with plasma, CSF concentrations or CSF-to-plasma ratios for either tenofovir or emtricitabine.

Keywords: pharmacokinetics; pharmacogenetics; tenofovir; emtricitabine; cerebrospinal fluid.

\section{Introduction}

Tenofovir and emtricitabine are part of the current first-line antiretroviral therapy (ART) regimens for HIV-positive adults in resource-limited settings and both are widely used in high-income countries. ${ }^{1}$ Infection of the central nervous system (CNS) by HIV-1 occurs early in infection and its clearance is reliant on adequate CNS antiretroviral concentrations. ${ }^{2}$ However, there are limited data regarding determinants of cerebrospinal fluid (CSF) penetration by tenofovir and emtricitabine. Data from small cohorts indicate that CSF concentrations of tenofovir and emtricitabine are 5\% and 50\% of plasma concentrations, respectively. $3,4,5$

However, higher CSF tenofovir concentrations and lower emtricitabine concentrations have been reported, which may be explained by polymorphisms in drug transporters or altered blood-brain barrier (BBB) permeability. ${ }^{4,5}$ Transporters in the BBB and blood-CSF barrier (BCB) affect the influx and efflux of drugs, including tenofovir and emtricitabine. ${ }^{3,6,7}$ Multidrug resistance protein-5 (MRP-5, encoded by ABCC5) is ubiquitous and mediates the efflux of nucleoside reverse transcriptase inhibitors. ${ }^{8}$ Lower CSF emtricitabine exposure in females compared to males is hypothesised to reflect differential expression of MRP transporters at the $\mathrm{BBB}$ and $\mathrm{BCB} .^{3}$ In vitro, tenofovir is a substrate of the breast cancer resistance protein (BCRP, encoded by $A B C G 2$ ), MRP-4 (encoded by $A B C C 4$ ) and P-glycoprotein (encoded by $A B C B 1) .9,10,11$ A polymorphism in $A B C G 2$ rs2231142 has been associated with 1.5-fold increased plasma tenofovir exposure and Thai patients carrying ABCC4 3463 AG or GG (rs1751034) had an 11\% greater tenofovir clearance compared with AA. ${ }^{12,13}$

How to cite this article: Decloedt EH, Sinxadi PZ, Wiesner L, Joska JA, Haas DW, Maartens G. Pharmacogenetics of tenofovir and emtricitabine penetration into cerebrospinal fluid. S Afr J HIV Med. 2021;22(1), a1206. https://doi.org/10.4102/sajhivmed.v22i1.1206 Copyright: @ 2021. The Authors. Licensee: AOSIS. This work is licensed under the Creative Commons Attribution License. 
Loss-of-function $A B C C 4$ polymorphisms have been associated with reduced clearance of tenofovir. ${ }^{11,14}$ In genome-wide analyses, SLC17A1 rs12662869 was associated with an increase in tenofovir clearance. ${ }^{15}$ It is possible that genetic polymorphisms that affect transporter function will affect tenofovir or emtricitabine CSF penetration. The pharmacogenetics of CSF penetration of tenofovir and emtricitabine have not been described.

Africans are the most genetically diverse population worldwide. ${ }^{16}$ South Africa has the world's largest ART programme, with most patients currently receiving efavirenz-based regimens that include the nucleos(t)ides tenofovir and emtricitabine. ${ }^{17}$ We previously reported on the pharmacogenetics of CSF penetration of efavirenz in black South Africans. ${ }^{18}$ Here, we characterise the associations between transporter gene polymorphisms and CSF penetration of tenofovir and emtricitabine in the same cohort.

\section{Patients and methods Participants}

Adults ( $\geq 18$ and $\leq 70$ years of age) from a randomised control trial (PACTR201310000635418) that investigated lithium for HIV-associated neurocognitive impairment were invited to participate in the present study. ${ }^{19}$ We also invited participants who were screened for that trial but were excluded based on cognitive impairment criteria. All participants provided written informed consent. This study was approved by the University of Cape Town Human Research Ethics Committee (HREC 071/2013).

\section{Pharmacokinetic sampling}

We collected paired plasma and CSF samples for tenofovir and emtricitabine assays. Participants recorded the dosing time the night before and were admitted in the morning for pharmacokinetic sampling. Whole blood was collected within 45 min of CSF sampling and centrifuged within $1 \mathrm{~h}$ of collection. Plasma and CSF aliquots were stored at $-80{ }^{\circ} \mathrm{C}$ until analysis.

\section{Tenofovir and emtricitabine measurement}

The analytical laboratory in the Division of Clinical Pharmacology at the University of Cape Town quantified total tenofovir and emtricitabine in plasma and CSF using validated liquid chromatography tandem mass spectrometry assays.

The lower limits of quantification (LLQs) for plasma tenofovir and emtricitabine were $10.0 \mathrm{ng} / \mathrm{mL}$ and $37.5 \mathrm{ng} / \mathrm{mL}$, respectively. For CSF, the LLQs for total tenofovir and emtricitabine were $0.5 \mathrm{ng} / \mathrm{mL}$. Concentrations below the limits of quantification were treated as missing data.

\section{Characterisation of genetic polymorphisms}

We extracted DNA from the buffy coat using the QIAsymphony kit. Genotyping was performed using the
Infinium ${ }^{\circledR}$ Expanded Multi-Ethnic Genotyping Array (MEGA $^{\mathrm{EX}}$; Illumina, San Diego, CA, USA).

Polymorphisms that were not genotyped were imputed. Polymorphisms were extracted from seven genes $\pm 50 \mathrm{kB}$ : $A B C B 1$ (301 polymorphisms), ABCC4 (630 polymorphisms), $A B C C 5$ (225 polymorphisms), ABCG2 (164 polymorphisms), SLCO1A2 (406 polymorphisms) and SLCO2B1 (118 polymorphisms). Polymorphisms were excluded for genotyping efficiency less than 99\%, minor allele frequency less than 5\% and Hardy-Weinberg equilibrium $p$-values less than 0.00001. We also genotyped SLCO1B1 521T $\rightarrow$ C (rs4149056) and SLCO1B1 (rs4149032) using the MassARRAY iPLEX® Gold system (Sequenom, Inc., San Diego, CA, USA).

All genotyping was performed at Vanderbilt Technologies for Advanced Genomics (VANTAGE), by laboratory personnel with no knowledge of clinical data. All samples were genotyped in duplicate. The final dataset included 1846 polymorphisms from 47 participants.

\section{Pharmacokinetic statistical analysis}

Pharmacokinetic data were not normally distributed so were expressed as median and interquartile ranges (IQRs) and geometric means (95\% confidence interval [CI]). Pearson's $r$ correlation was used to assess the correlations between plasma and CSF concentrations. We performed statistical analysis using STATA version 15.0 (StataCorp, College Station, TX, USA). Graphs were created using GraphPad Prism version 7.03 for Windows (GraphPad Software, La Jolla, CA, USA).

\section{Genetic associations}

Associations with pharmacokinetic parameters were assessed by univariable analysis. Pharmacokinetic data were $\log _{10}$ transformed for association analyses. We used ratios of total concentrations without correcting for protein binding. Cerebrospinal fluid-to-plasma concentration ratios were calculated using raw concentrations and then $\log _{10}$ transformed. We performed genetic association analyses using PLINK version 1.9. ${ }^{20}$

For primary analyses, we conducted linkage disequilibrium (LD) pruned with an $\mathrm{R}^{2}$ threshold of 0.95 within a $50-\mathrm{kB}$ window at $5-\mathrm{kB}$ increments.

The final analysis included 782 polymorphisms that met the LD-pruning threshold. We used Bonferroni correction to adjust for multiple testing $(p=0.05$ divided by 782 polymorphisms). We generated an LD plot using Haploview (https://www.broadinstitute.org/haploview/haploview). We previously reported LD plots for these polymorphisms. ${ }^{18}$

\section{Ethical considerations}

All participants provided written informed consent. This study was approved by the University of Cape Town Human Research Ethics Committee (HREC 071/2013). 


\section{Results}

We studied 47 participants who self-identified as black South Africans (isiXhosa speaking), of whom 41 were female. All were virologically suppressed and were receiving efavirenz, tenofovir and emtricitabine $(n=43)$ or efavirenz, tenofovir and lamivudine $(n=4)$. The median (IQR) values of the baseline characteristics were age $36(\mathrm{IQR}=32-43)$ years, a $\mathrm{CD}_{4}$ T-cell count of $470(\mathrm{IQR}=384-586)$ cells $/ \mathrm{mm}^{3}$, a time on ART of 38 (IQR = 18-54) months and a body mass index (BMI) of $25.6(\mathrm{IQR}=22.7-29.3) \mathrm{kg} / \mathrm{m}^{2}$. The concentrations of tenofovir (plasma and CSF) and emtricitabine (plasma and CSF) are presented in Table 1. The plasma and CSF concentrations of tenofovir and emtricitabine were each correlated $\left(p<0.0001, \mathrm{R}^{2}=0.53\right.$ and $p<0.0001, \mathrm{R}^{2}=0.45$; respectively) (Appendix Figure $1 \mathrm{a}$ and $1 \mathrm{~b}$ ). There was no statistically significant association of CSF-to-plasma ratios versus time after dosing (Appendix Figure 2).

\section{Genetic polymorphisms}

Amongst the 47 participants, 1846 polymorphisms were successfully genotyped. Only SLCO1B1 rs4149056 was monomorphic (i.e. no minor alleles). The remaining 1845 polymorphisms were in Hardy-Weinberg equilibrium based on a Bonferroni-adjusted $p$-value threshold of $6.4 \times 10^{-5}$; 56 had unadjusted Bonferroni $p$-values of $<0.05$. Minor allele frequencies for all polymorphisms are provided in Appendix Table 1.

\section{Genetic associations with tenofovir and emtricitabine cerebrospinal fluid penetration}

In univariable linear regression analyses (Table 2), the tenofovir CSF-to-plasma ratio was best predicted by a model that included $A B C B 1$ rs1989830 ( $\beta=-0.12 ; 95 \%$ $\left.\mathrm{CI}=-0.19--0.05 ; p=1.2 \times 10^{-3}\right)$. The emtricitabine CSF-toplasma ratio was best predicted by a model that included $A B C C 5$ rs11921035 ( $\beta=-0.32 ; 95 \%$ CI $=-0.50-$ $\left.-0.14 ; p=1.4 \times 10^{-3}\right)$, as shown in Table 3 . No association achieved significance after correcting for multiple testing. Univariable linear regression analyses and polymorphisms with $p$-values below 0.01 for tenofovir and emtricitabine CSF-to-plasma ratios are shown in Tables 2 and 3, respectively. For absolute plasma and CSF tenofovir concentrations, 10 polymorphisms in ABCG2, ABCC5, SLCO1A2 and $A B C C 4$ for plasma and six in $A B C B 1, A B C G 2$, $A B C C 5, S L C O 1 A 2$ and $A B C C 4$ for CSF had $p$-values less than 0.01 (data not shown). For absolute plasma and CSF emtricitabine concentrations, six polymorphisms in $A B C C 5$, SLCO1A2, ABCC4 and SLCO2B1 for plasma and 12 in ABCB1, $A B C G 2, A B C C 5, S L C O 1 A 2$ and $A B C C 4$ had $p$-values less than 0.01 (data not shown). No associations with SLCO1B1 rs4149032 were found.

\section{Discussion}

We characterised the associations between 782 genetic polymorphisms and CSF disposition of tenofovir and emtricitabine in black South African adults. The lowest

TABLE 1: Concentrations of tenofovir and emtricitabine in plasma and cerebrospinal fluid.

\begin{tabular}{|c|c|c|c|c|c|c|c|c|c|c|c|c|}
\hline \multirow[t]{3}{*}{ Nucleos(t)ides } & \multicolumn{12}{|c|}{ Data for } \\
\hline & \multicolumn{4}{|c|}{ Plasma } & \multicolumn{4}{|c|}{ CSF } & \multicolumn{4}{|c|}{ CSF-to-plasma ratio } \\
\hline & Value & IQR & $\%$ & CI & Value & IQR & $\%$ & CI & Value & IQR & $\%$ & CI \\
\hline \multicolumn{13}{|l|}{ Tenofovir $(n=47)$} \\
\hline BLQ samples, $n(\%)$ & 3 & - & 6.4 & - & 4 & - & 8.5 & - & 4 & - & 8.5 & - \\
\hline Median concentration, $\mathrm{ng} / \mathrm{mL}$ (IQR) & 63.5 & $50.8-81.2$ & - & - & 1.4 & $1.1-2.1$ & - & - & 0.023 & $0.018-0.030$ & - & - \\
\hline Geometric mean, $\mathrm{ng} / \mathrm{mL}(95 \% \mathrm{Cl})$ & 62.57 & - & - & $53.91-72.63$ & 1.49 & - & - & $1.28-1.73$ & 0.023 & - & - & $0.021-0.026$ \\
\hline Range, $\mathrm{ng} / \mathrm{mL}$ & $23-246$ & - & - & - & $0.51-5.3$ & - & - & - & $0.012-0.047$ & - & - & - \\
\hline \multicolumn{13}{|l|}{ Emtricitabine $(n=43)$} \\
\hline BLQ samples, $n(\%)$ & 4 & - & 17 & - & 4 & - & 9 & - & 4 & - & 17 & - \\
\hline Median concentration, $\mathrm{ng} / \mathrm{mL}$ (IQR) & 139 & $109-166$ & - & - & 63.5 & $47.4-102$ & - & - & 0.534 & $0.392-0.675$ & - & - \\
\hline Geometric mean, ng/mL $(95 \% \mathrm{Cl})$ & 133.7 & - & - & 112.1-159.4 & 56.0 & - & - & $41.7-75.0$ & 0.528 & - & - & $0.460-0.605$ \\
\hline Range, ng/mL & $39.3-560$ & - & - & - & $1.5-167$ & - & - & - & $0.207-2.007$ & - & - & - \\
\hline
\end{tabular}

CSF, cerebrospinal fluid; IQR, interquartile range; $95 \% \mathrm{Cl}, 95 \%$ confidence interval; BLQ, below limits of assay quantification.

TABLE 2: Genetic associations with detectable log10-transformed cerebrospinal fluid-to-plasma tenofovir concentrations in 43 black South African adults.

\begin{tabular}{|c|c|c|c|c|c|c|}
\hline Chromosome & Gene & Polymorphism & Minor allele frequency & Beta coefficient & $95 \% \mathrm{Cl}$ & $p^{*}$ \\
\hline 7 & $A B C B 1$ & rs1989830 & 0.30 & -0.12 & $-0.19--0.05$ & $1.2 \times 10^{-3}$ \\
\hline 7 & $A B C B 1$ & rs78551545 & 0.06 & -0.28 & $-0.43--0.12$ & $1.3 \times 10^{-3}$ \\
\hline 12 & SLCO1A2 & rs11535999 & 0.33 & 0.13 & $0.05-0.21$ & $2.5 \times 10^{-3}$ \\
\hline 4 & $A B C G 2$ & rs111917717 & 0.05 & -0.28 & $-0.46--0.11$ & $3.0 \times 10^{-3}$ \\
\hline 4 & $A B C G 2$ & rs76462878 & 0.08 & -0.22 & $-0.36--0.08$ & $4.0 \times 10^{-3}$ \\
\hline 7 & $A B C B 1$ & rs35572298 & 0.08 & -0.18 & $-0.30--0.06$ & $5.0 \times 10^{-3}$ \\
\hline 12 & SLCO1A2 & rs4149008 & 0.38 & 0.11 & $0.03-0.18$ & $6.9 \times 10^{-3}$ \\
\hline 12 & SLCO1A2 & rs4149009 & 0.32 & 0.11 & $0.03-0.19$ & $7.8 \times 10^{-3}$ \\
\hline 12 & SLCO1A2 & rs10841786 & 0.33 & 0.11 & $0.03-0.19$ & $7.8 \times 10^{-3}$ \\
\hline 12 & SLCO1A2 & rs57472326 & 0.33 & 0.11 & $0.03-0.19$ & $7.8 \times 10^{-3}$ \\
\hline 12 & SLCO1A2 & rs7968842 & 0.34 & 0.11 & $0.03-0.19$ & $9.2 \times 10^{-3}$ \\
\hline
\end{tabular}

$95 \% \mathrm{Cl}, 95 \%$ confidence interval.

$*$, Bonferroni-corrected $p$-value cut-off $=6.4 \times 10^{-5}$. 
TABLE 3: Genetic associations with detectable $\log _{10}$-transformed cerebrospinal fluid -to plasma emtricitabine concentrations in 39 South African adults.

\begin{tabular}{|c|c|c|c|c|c|c|}
\hline Chromosome & Gene & Polymorphism & Minor allele frequency & Beta coefficient & $95 \% \mathrm{Cl}$ & $p^{*}$ \\
\hline 3 & $A B C C 5$ & rs11921035 & 0.08 & -0.32 & $-0.50--0.14$ & $1.4 \times 10^{-3}$ \\
\hline 12 & SLCO1A2 & rs4762700 & 0.48 & -0.16 & $-0.25--0.06$ & $2.0 \times 10^{-3}$ \\
\hline 3 & $A B C C 5$ & rs11928606 & 0.09 & -0.27 & $-0.44--0.10$ & $4.4 \times 10^{-3}$ \\
\hline 13 & $A B B C 4$ & rs7322318 & 0.34 & 0.13 & $0.05-0.22$ & $5.3 \times 10^{-3}$ \\
\hline 13 & $A B C C 4$ & rs9590228 & 0.38 & 0.13 & $0.04-0.22$ & $6.5 \times 10^{-3}$ \\
\hline 13 & $A B C C 4$ & rs4148428 & 0.21 & -0.18 & $-0.30--0.06$ & $7.4 \times 10^{-3}$ \\
\hline 3 & $A B C C 5$ & rs116312201 & 0.13 & 0.19 & $0.06-0.33$ & $9.0 \times 10^{-3}$ \\
\hline 4 & $A B C G 2$ & rs1448784 & 0.05 & -0.32 & $-0.54--0.09$ & $9.3 \times 10^{-3}$ \\
\hline
\end{tabular}

$95 \% \mathrm{Cl}, 95 \%$ confidence interval.

$*$, Bonferroni-corrected $p$-value cut-off $=6.4 \times 10^{-5}$.

$p$-value for tenofovir CSF-to-plasma ratio was $A B C B 1$ rs1989830 $\left(p=1.2 \times 10^{-3}\right)$, and for emtricitabine was ABCC5 rs11921035 $\left(p=1.4 \times 10^{-3}\right)$. None were significant after correcting for multiple testing. In addition, we found no significant associations with absolute CSF or plasma concentration after correcting for multiple testing.

Associations with tenofovir pharmacokinetics and genetic polymorphisms were found in other populations. An increase in tenofovir plasma concentrations were independently associated with $A B C C 44131 \mathrm{~T} \rightarrow \mathrm{G}$ (genotype TG or GG) in 150 Thai HIV-infected adults. ${ }^{14} A B C B 1$ rs3213619 was associated with increased tenofovir bioavailability in a predominantly African-American patient population $(n=45)$ and thought to be a result of decreased P-glycoprotein function. ${ }^{21}$ A genomewide and candidate gene association analyses with tenofovir pharmacokinetics showed that SLC17A1 rs12662869 was associated with an increase in tenofovir clearance $\left(p=7.1 \times 10^{-9}\right)$ but failed to show significant associations in candidate genes (including $A B C C 4, A B C C 10, A B C B 1, A B C C 2, S L C 22 A 11, A K 2$ and $A K 3$ ) after correction for multiple comparisons. ${ }^{15}$

Our study has limitations. With our sample size, we were underpowered to detect associations with small effect sizes. We could only detect associations with relatively frequent polymorphisms and with large effect sizes. Therefore, these data should be regarded as exploratory. Polymorphisms not genotyped in our study may be associated with tenofovir or emtricitabine disposition into CSF. Whilst we did not adjust for creatinine clearance, this should not be a confounder that affects drug disposition into CSF. We included $33(70 \%)$ participants with mild to moderate neurocognitive impairment, as previously reported..$^{18} \mathrm{We}$ may therefore have introduced a selection bias.

\section{Conclusion}

In conclusion, we found no significant associations between any of the 782 polymorphisms and plasma concentrations, CSF concentrations or CSF-to-plasma ratios for either tenofovir or emtricitabine in univariate linear regression models after correcting for multiple testing.

\section{Acknowledgements}

The authors are grateful to the study participants. The study team in alphabetical order includes Laura Comrie (clinical support), Carla Freeman (clinical support), Shahieda Isaacs (viral sequencing), Pam Jordan (data capturer), Teboho Linda (neuropsychology technician). Nozipho Mawisa (study nurse), Queen Maswana (recruiter), Rasmita Ori (clinical support), Kareema Poggenpoel (administration support) and Shireen Surtie (study coordinator). The authors are also grateful to Cara Sutcliffe and the VANTAGE team at Vanderbilt.

\section{Competing interests}

The authors declare that they have no financial or personal relationships that may have inappropriately influenced them in writing this article.

\section{Authors' contributions}

E.H.D. was responsible for the study concept and design, data acquisition, data analysis and interpretation of data, drafting and revising the manuscript for content, study supervision and obtaining funding. P.Z.S. contributed to data analysis and interpretation of data and drafted and revised the manuscript for content. L.W. conducted the sample analysis and revised the manuscript for content. J.A.J. contributed to the study concept and design, revised the manuscript for content and supervised the study. D.W.H. conducted the sample analysis and data analysis, interpreted the data and revised the manuscript for content. G.M. contributed to the study concept and design and revised the manuscript for content.

\section{Funding information}

This work was supported by the South African Medical Research Council and the European and Developing Countries Clinical Trials Partnership (SP.2011.41304.065). The drug assays analysed at the University of Cape Town were supported in part by the National Institute of Allergy and Infectious Diseases of the National Institutes of Health (UM1 AI068634, UM1 AI068636, UM1 AI106701 and U01 AI068632), the Eunice Kennedy Shriver National Institute of Child Health and Human Development (NICHD) and the National Institute of Mental Health (AI068632). D.W.H. is supported by National Institutes of Health grants AI077505, AI069439 and TR002243.

\section{Data availability}

The data that support the findings of this study are available from the corresponding author, E.H.D., upon reasonable request. 


\section{Disclaimer}

The views and opinions expressed in this article are those of the authors and do not necessarily reflect the official policy or position of any affiliated agency of the authors.

\section{References}

1. World Health Organization (WHO). First-line ART for adults [homepage on the Internet]. [cited 2017 Jun 1]. Available from: http://www.who.int/hiv/pub/ guidelines/arv2013/art/artadults/en/

2. Valcour V, Chalermchai T, Sailasuta N, et al. Central nervous system viral invasion and inflammation during acute HIV infection. J Infect Dis. 2012;206(2):275-282. and inflammation during acute HIV infe
https://doi.org/10.1093/infdis/jis326

3. Lahiri CD, Reed-Walker K, Sheth AN, Acosta EP, Vunnava A, Ofotokun I. Cerebrospinal fluid concentrations of tenofovir and emtricitabine in the setting of HIV-1 protease inhibitor-based regimens. J Clin Pharmacol. 2016;56(4):492-496. https://doi.org/10.1002/jcph.612

4. Best BM, Letendre SL, Koopmans P, et al. Low CSF concentrations of the nucleotide HIV reverse transcriptase inhibitor, tenofovir. J Acquir Immune Defic Syndr. 2013;59(4):376-381. https://doi.org/10.1097/QAl.0b013e318247ec54

5. Calcagno A, Bonora S, Simiele $M$, et al. Tenofovir and emtricitabine cerebrospinal fluid-to-plasma ratios correlate to the extent of blood-brainbarrier damage. AIDS. 2011;25(11):1437-1439. https://doi.org/10.1097/QAD.0b013e3283489cb1

6. Suhy AM, Webb A, Papp AC, Geier EG, Sadee W. Expression and splicing of $A B C$ and SLC transporters in the human blood-brain barrier measured with RNAseq
Eur J Pharm Sci. 2017;103:47-51. https://doi.org/10.1016/j.ejps.2017.02.010

7. Varatharajan L, Thomas SA. The transport of anti-HIV drugs across blood-CNS interfaces: Summary of current knowledge and recommendations for further research. Antiviral Res. 2009;82(2):A99-A109. https://doi.org/10.1016/j.antiviral. 2008.12.013

8. Reid G, Wielinga P, Zelcer N, et al. Characterization of the transport of nucleoside analog drugs by the human multidrug resistance proteins MRP4 and MRP5. Mo Pharmacol. 2003;63(5):1094-1103. https://doi.org/10.1124/mol.63.5.1094

9. Reznicek J, Ceckova M, Cerveny L, Müller F, Staud F. Emtricitabine is a substrate of MATE1 but not of OCT1, OCT2, P-gp, BCRP or MRP2 transporters. Xenobiotica. 2017;47(1):77-85. https://doi.org/10.3109/00498254.2016.1158886

10. Neumanova Z, Cerveny L, Ceckova M, Staud F. Interactions of tenofovir and tenofovir disoproxil fumarate with drug efflux transporters ABCB1, ABCG2, and org/10.1097/QAD.0000000000000112
11. Imaoka T, Kusuhara H, Adachi M, Schuetz JD, Takeuchi K, Sugiyama Y. Functional involvement of multidrug resistance-associated protein 4 (MRP4/ABCC4) in the renal elimination of the antiviral drugs adefovir and tenofovir. Mol Pharmacol. 2007;71(2):619-627. https://doi.org/10.1124/mol.106.028233

12. Rungtivasuwan K, Avihingsanon A, Thammajaruk N, et al. Pharmacogeneticsbased population pharmacokinetic analysis of tenofovir in Thai HIV-infected patients. Pharmacogenomics J. 2017;18(16):1481-1490. https://doi.org/10.2217/ pgs-2017-0128

13. Baxi SM, Greenblatt RM, Bacchetti $P$, et al. Evaluating the association of singlenucleotide polymorphisms with tenofovir exposure in a diverse prospective cohort of women living with HIV. Pharmacogenomics J. 2017;18:245-250. https:// doi.org/10.1038/tpj.2017.3

14. Rungtivasuwan $K$, Avihingsanon A, Thammajaruk N, et al. Influence of $A B C C 2$ and ABCC4 polymorphisms on tenofovir plasma concentrations in Thai HIV-infected patients. Antimicrob Agents Chemother. 2015;59(6):3240-3245. https://doi. org/10.1128/AAC.04930-14

15. Wanga V, Venuto C, Morse GD, et al. Genomewide association study of tenofovir pharmacokinetics and creatinine clearance in AIDS clinical trials group protocol A5202. Pharmacogenet Genomics. 2015;25(9):450-461. https://doi.org/10.1097/ FPC.0000000000000156

16. Campbell MC, Tishkoff SA. African genetic diversity: limplications for human demographic history, modern human origins, and complex disease mapping. Annu Rev Genomics Hum Genet. 2008;9:403-433. https://doi.org/10.1146/ annurev.genom.9.081307.164258

17. World Health Organization. Antiretroviral therapy coverage data and estimates by country [homepage on the Internet]. Global Health Observatory Data Repository; 2017 [cited 2018 Apr 23]. Available from: http://apps.who.int/gho/data/node. main.626?lang=en 2017

18. Decloedt EH, Sinxadi PZ, Van Zyl GU, et al. Pharmacogenetics and pharmacokinetics of CNS penetration of efavirenz and its metabolites. J Antimicrob Chemother 2019;74(3):699-709. https://doi.org/10.1093/jac/dky481

19. Decloedt EH, Freeman C, Howells F, et al. Moderate to severe HIV-associated neurocognitive impairment: A randomized placebo-controlled trial of lithium. Medicine. 2016;95(46):e5401. https://doi.org/10.1097/MD.0000000000005401

20. Purcell S, Neale B, Todd-Brown K, et al. PLINK: A tool set for whole-genome association and population-based linkage analyses. Am J Hum Genet. 2007;81(3):559-575.

21. Chen J, Akhtari FS, Wagner MJ, et al. Pharmacogenetic analysis of the modelbased pharmacokinetics of five anti-HIV drugs: How does this influence the effect of aging? Clin Transl Sci. 2018;11(2):226-236. https://doi.org/10.1111/ cts. 12525 


\section{Appendix}

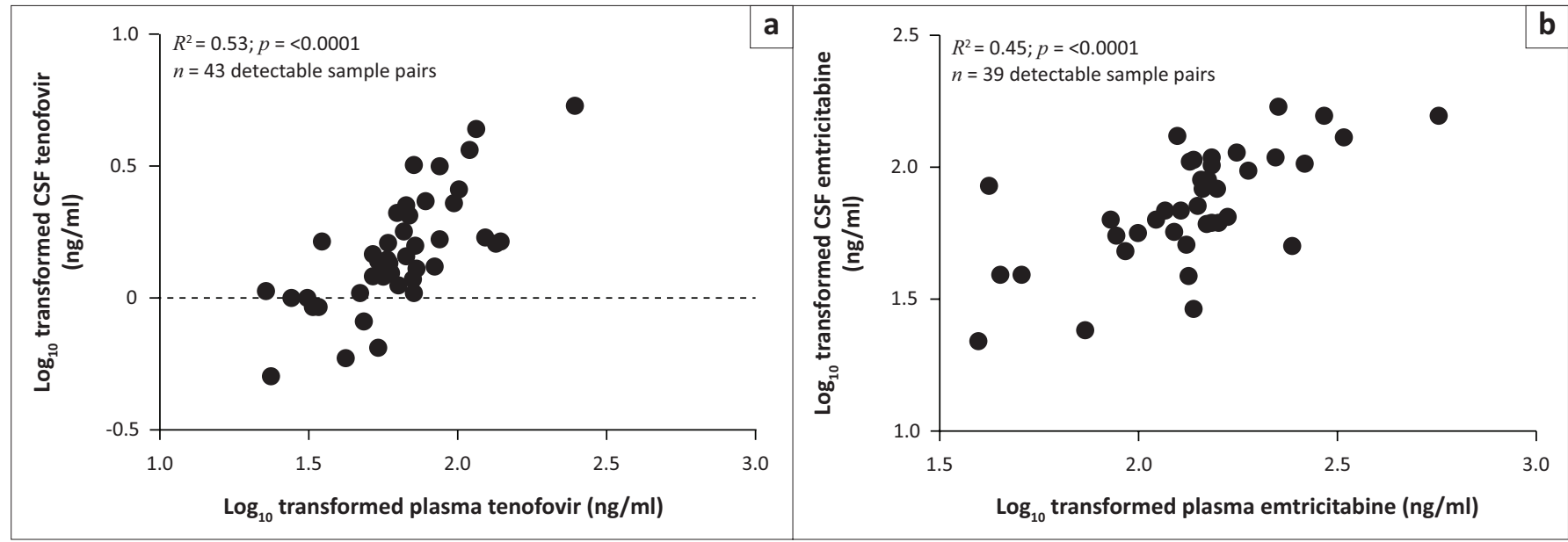

Figure 1-A1: Pearson correlation plots for $\log _{10}$-transformed plasma and cerebrospinal fluid (CSF) concentrations emtricitabine and tenofovir. Panel A: Relationship between CSF and plasma tenofovir concentrations. Panel B: Relationship between CSF and plasma emtricitabine concentrations. All concentrations are log ${ }_{10}$ transformed.

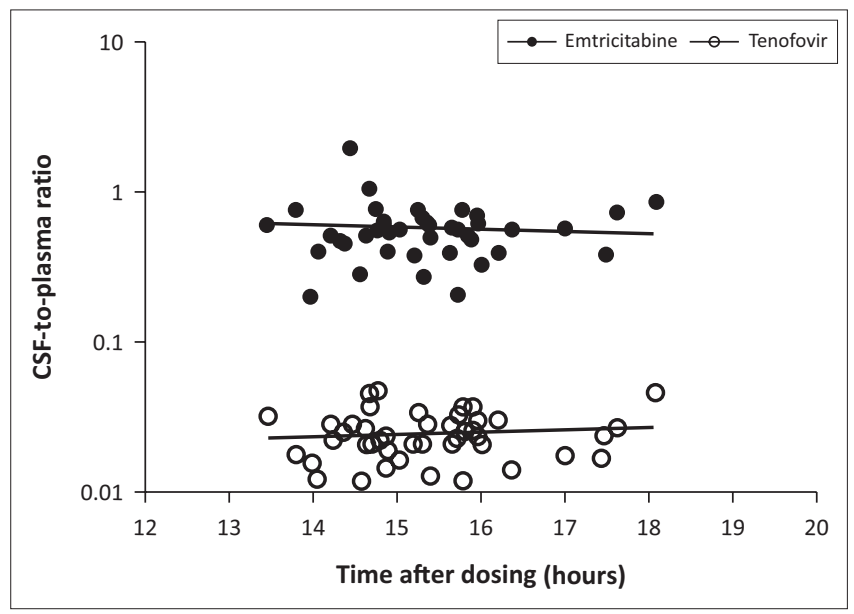

Figure 2-A1: Cerebrospinal fluid (CSF)-to-plasma concentration ratios of detectable pairs of plasma and CSF samples versus time after dosing. The lines are linear regression lines and were not statistically significant. 
Table 1-A1: Minor allele frequencies for 1846 polymorphisms in 43 black South Africans.

\begin{tabular}{|c|c|c|c|c|}
\hline Chromosome & Polymorphism & Minor allele & Major allele & $\begin{array}{l}\text { Minor allele } \\
\text { frequency }\end{array}$ \\
\hline 3 & rs73887541 & C & G & 0.1744 \\
\hline 3 & rs142034818 & A & G & 0.1744 \\
\hline 3 & rs11928605 & $\mathrm{T}$ & C & 0.0697 \\
\hline 3 & rs11928606 & $\mathrm{T}$ & C & 0.0930 \\
\hline 3 & rs6443917 & $\mathrm{T}$ & C & 0.3721 \\
\hline 3 & rs11921035 & G & A & 0.0814 \\
\hline 3 & rs11914385 & $\mathrm{T}$ & C & 0.0814 \\
\hline 3 & rs11914437 & A & G & 0.0930 \\
\hline 3 & rs6776999 & G & $\mathrm{T}$ & 0.3605 \\
\hline 3 & rs115851049 & A & $\mathrm{T}$ & 0.1744 \\
\hline 3 & rs7635679 & $\mathrm{T}$ & C & 0.4535 \\
\hline 3 & rs7648408 & A & $\mathrm{T}$ & 0.3605 \\
\hline 3 & rs7648487 & A & G & 0.3605 \\
\hline 3 & rs113227666 & $\mathrm{T}$ & C & 0.0930 \\
\hline 3 & rs12696511 & A & G & 0.1279 \\
\hline 3 & rs111485412 & $\mathrm{T}$ & C & 0.0814 \\
\hline 3 & rs5855004 & AT & A & 0.3721 \\
\hline 3 & rs9290776 & C & A & 0.3605 \\
\hline 3 & rs73044605 & A & G & 0.0814 \\
\hline 3 & rs188783928 & C & $\mathrm{T}$ & 0.0814 \\
\hline 3 & rs149185640 & A & AAGAG & 0.0814 \\
\hline 3 & rs75086702 & $\mathrm{T}$ & C & 0.1744 \\
\hline 3 & rs11926985 & A & G & 0.2326 \\
\hline 3 & rs56772648 & G & C & 0.1744 \\
\hline 3 & rs56333370 & G & A & 0.1744 \\
\hline 3 & rs 35494670 & G & $\mathrm{T}$ & 0.4419 \\
\hline 3 & rs 35717944 & A & C & 0.2558 \\
\hline 3 & rs6792309 & $\mathrm{T}$ & C & 0.4767 \\
\hline 3 & rs75822913 & A & G & 0.0930 \\
\hline 3 & rs1879256 & G & A & 0.4884 \\
\hline 3 & rs1879255 & A & C & 0.314 \\
\hline 3 & rs1879254 & $\mathrm{T}$ & $C$ & 0.3023 \\
\hline 3 & rs56874920 & $\mathrm{T}$ & TA & 0.2093 \\
\hline 3 & rs73887563 & A & G & 0.1744 \\
\hline 3 & rs73887565 & A & G & 0.186 \\
\hline 3 & rs2139558 & G & A & 0.3953 \\
\hline 3 & rs35369718 & G & A & 0.3953 \\
\hline 3 & rs17683012 & $\mathrm{T}$ & C & 0.3953 \\
\hline 3 & rs1012987 & G & $\mathrm{T}$ & 0.3953 \\
\hline 3 & rs1012988 & A & G & 0.3953 \\
\hline 3 & rs1012989 & $\mathrm{T}$ & G & 0.3953 \\
\hline 3 & rs872013 & A & G & 0.2442 \\
\hline 3 & rs73044669 & C & $\mathrm{T}$ & 0.2326 \\
\hline 3 & rs7434228 & $\mathrm{T}$ & C & 0.2558 \\
\hline 3 & rs2176825 & A & G & 0.2558 \\
\hline 3 & rs1533682 & $\mathrm{T}$ & $C$ & 0.2326 \\
\hline 3 & rs1533683 & C & $\mathrm{T}$ & 0.3488 \\
\hline 3 & rs73044685 & $\mathrm{T}$ & G & 0.0581 \\
\hline 3 & rs 1000002 & $T$ & C & 0.1744 \\
\hline 3 & rs61698626 & $\mathrm{T}$ & $C$ & 0.2907 \\
\hline 3 & rs1533684 & A & G & 0.2326 \\
\hline 3 & rs2313217 & A & G & 0.2326 \\
\hline 3 & rs6768149 & C & $\mathrm{T}$ & 0.1744 \\
\hline 3 & rs2872249 & $\mathrm{T}$ & A & 0.2442 \\
\hline 3 & rs79625907 & $\mathrm{T}$ & $C$ & 0.1047 \\
\hline 3 & rs73044690 & C & A & 0.0930 \\
\hline 3 & rs3805114 & G & $\mathrm{T}$ & 0.1047 \\
\hline 3 & rs562 & $\mathrm{T}$ & C & 0.3372 \\
\hline 3 & rs3749445 & $\mathrm{T}$ & C & 0.1744 \\
\hline 3 & rs73884807 & A & G & 0.2442 \\
\hline
\end{tabular}

Table 1-A1 continues in the next column $\rightarrow$
Table 1-A1 (Continues...): Minor allele frequencies for 1846 polymorphisms in 43 black South Africans.

\begin{tabular}{|c|c|c|c|c|}
\hline Chromosome & Polymorphism & Minor allele & Major allele & $\begin{array}{l}\text { Minor allele } \\
\text { frequency }\end{array}$ \\
\hline 3 & rs60053269 & G & GA & 0.2326 \\
\hline 3 & rs112357545 & $T$ & C & 0.0581 \\
\hline 3 & rs7646621 & G & $\mathrm{T}$ & 0.4767 \\
\hline 3 & rs77093210 & A & G & 0.1047 \\
\hline 3 & rs13317532 & G & C & 0.2442 \\
\hline 3 & rs113699660 & C & G & 0.0581 \\
\hline 3 & rs113422085 & $T$ & C & 0.0697 \\
\hline 3 & rs111666830 & C & $\mathrm{T}$ & 0.0581 \\
\hline 3 & rs113311136 & C & $\mathrm{T}$ & 0.0581 \\
\hline 3 & rs2139562 & G & A & 0.2907 \\
\hline 3 & rs4148593 & G & A & 0.5 \\
\hline 3 & rs6766986 & C & G & 0.2442 \\
\hline 3 & rs112840402 & $\mathrm{T}$ & C & 0.0581 \\
\hline 3 & rs116312201 & A & G & 0.1279 \\
\hline 3 & rs147028569 & $\mathrm{T}$ & C & 0.0581 \\
\hline 3 & rs113115970 & $\mathrm{T}$ & C & 0.0581 \\
\hline 3 & rs11714326 & $\mathrm{T}$ & C & 0.2209 \\
\hline 3 & rs13064234 & $A$ & G & 0.2209 \\
\hline 3 & rs113010279 & C & $\mathrm{T}$ & 0.0581 \\
\hline 3 & rs6790814 & G & C & 0.2093 \\
\hline 3 & rs111868993 & C & $T$ & 0.0581 \\
\hline 3 & rs111248225 & $\mathrm{T}$ & C & 0.0581 \\
\hline 3 & rs6443922 & G & A & 0.2209 \\
\hline 3 & rs6804242 & A & $\mathrm{T}$ & 0.2326 \\
\hline 3 & rs59518404 & A & G & 0.2209 \\
\hline 3 & rs115503048 & G & $\mathrm{T}$ & 0.0581 \\
\hline 3 & rs3749442 & A & G & 0.2209 \\
\hline 3 & rs73884809 & G & $\mathrm{T}$ & 0.2442 \\
\hline 3 & rs73046532 & C & G & 0.1047 \\
\hline 3 & rs10575785 & G & GAAC & 0.2093 \\
\hline 3 & rs73046540 & C & $T$ & 0.1279 \\
\hline 3 & rs6443923 & A & C & 0.3488 \\
\hline 3 & rs6805913 & $\mathrm{T}$ & G & 0.2326 \\
\hline 3 & rs9812777 & $T$ & C & 0.2093 \\
\hline 3 & rs4148589 & C & $T$ & 0.2209 \\
\hline 3 & rs2292998 & $\mathrm{T}$ & C & 0.2326 \\
\hline 3 & rs9818518 & $\mathrm{T}$ & C & 0.2209 \\
\hline 3 & rs9838667 & G & $T$ & 0.3488 \\
\hline 3 & rs3840256 & GAT & G & 0.2209 \\
\hline 3 & rs6799583 & C & G & 0.2209 \\
\hline 3 & rs3817404 & A & C & 0.1628 \\
\hline 3 & rs189855476 & C & $\mathrm{T}$ & 0.0581 \\
\hline 3 & rs2139564 & A & G & 0.2209 \\
\hline 3 & rs111952578 & C & G & 0.0581 \\
\hline 3 & rs59309690 & A & G & 0.0930 \\
\hline 3 & rs115663763 & A & $\mathrm{T}$ & 0.0581 \\
\hline 3 & rs116483375 & $\mathrm{T}$ & C & 0.0581 \\
\hline 3 & rs1401999 & C & G & 0.2209 \\
\hline 3 & rs6443924 & A & G & 0.2442 \\
\hline 3 & rs149330260 & A & G & 0.0581 \\
\hline 3 & rs6766401 & $\mathrm{T}$ & C & 0.2209 \\
\hline 3 & rs6443925 & C & G & 0.4535 \\
\hline 3 & rs6766526 & $T$ & C & 0.2209 \\
\hline 3 & rs6767013 & G & $A$ & 0.2209 \\
\hline 3 & rs187791141 & C & $T$ & 0.0581 \\
\hline 3 & rs6767306 & A & G & 0.2209 \\
\hline 3 & rs1879257 & A & G & 0.2209 \\
\hline 3 & rs3792583 & A & G & 0.2209 \\
\hline 3 & rs4148579 & $T$ & C & 0.2209 \\
\hline 3 & rs939336 & A & G & 0.2442 \\
\hline
\end{tabular}

Table 1-A1 continues on the next page $\rightarrow$ 
Table 1-A1 (Continues...): Minor allele frequencies for 1846 polymorphisms in 43 black South Africans.

\begin{tabular}{|c|c|c|c|c|}
\hline Chromosome & Polymorphism & Minor allele & Major allele & $\begin{array}{l}\text { Minor allele } \\
\text { frequency }\end{array}$ \\
\hline 3 & rs6800217 & $\mathrm{T}$ & C & 0.2442 \\
\hline 3 & rs35240483 & A & C & 0.2209 \\
\hline 3 & rs112737137 & $\mathrm{T}$ & C & 0.2209 \\
\hline 3 & rs7636305 & A & G & 0.2209 \\
\hline 3 & rs9861983 & $\mathrm{T}$ & C & 0.2209 \\
\hline 3 & rs869417 & $\mathrm{T}$ & C & 0.2209 \\
\hline 3 & rs28365012 & A & G & 0.0581 \\
\hline 3 & rs3828469 & G & A & 0.2209 \\
\hline 3 & rs3805108 & $\mathrm{T}$ & C & 0.3372 \\
\hline 3 & rs73179688 & $\mathrm{T}$ & $\mathrm{C}$ & 0.4419 \\
\hline 3 & rs144452121 & C & $\mathrm{T}$ & 0.0581 \\
\hline 3 & rs113468175 & C & G & 0.0581 \\
\hline 3 & rs144412198 & A & G & 0.0581 \\
\hline 3 & rs3792581 & A & $\mathrm{C}$ & 0.2209 \\
\hline 3 & rs114398776 & G & A & 0.0581 \\
\hline 3 & rs4148578 & A & $\mathrm{C}$ & 0.2209 \\
\hline 3 & rs4148577 & C & $\mathrm{T}$ & 0.2209 \\
\hline 3 & rs1132776 & A & G & 0.3372 \\
\hline 3 & rs6775518 & A & G & 0.2209 \\
\hline 3 & rs6791345 & $\mathrm{T}$ & C & 0.3372 \\
\hline 3 & rs6802849 & A & C & 0.2209 \\
\hline 3 & rs55695073 & $\mathrm{T}$ & C & 0.2093 \\
\hline 3 & rs7636910 & C & $\mathrm{T}$ & 0.186 \\
\hline 3 & rs2293001 & $\mathrm{T}$ & C & 0.4419 \\
\hline 3 & rs2313212 & A & G & 0.2442 \\
\hline 3 & rs939337 & G & C & 0.2209 \\
\hline 3 & rs75617395 & $\mathrm{T}$ & C & 0.0930 \\
\hline 3 & rs3749440 & G & A & 0.4419 \\
\hline 3 & rs4148575 & A & G & 0.3372 \\
\hline 3 & rs6795595 & $\mathrm{T}$ & C & 0.2558 \\
\hline 3 & rs57777233 & $\mathrm{T}$ & C & 0.2558 \\
\hline 3 & rs3749438 & A & G & 0.186 \\
\hline 3 & rs112403322 & G & C & 0.0581 \\
\hline 3 & rs6443926 & A & C & 0.3372 \\
\hline 3 & rs73884816 & G & A & 0.2558 \\
\hline 3 & rs7635948 & C & G & 0.3372 \\
\hline 3 & rs10937157 & A & G & 0.2791 \\
\hline 3 & rs12634398 & G & A & 0.4419 \\
\hline 3 & rs10937158 & $\mathrm{T}$ & C & 0.2791 \\
\hline 3 & rs113691647 & A & ACAAAAGTGCATG & 0.2096 \\
\hline 3 & rs11404217 & GA & G & 0.3953 \\
\hline 3 & rs75393197 & G & $\mathrm{T}$ & 0.0581 \\
\hline 3 & rs28680881 & G & $\mathrm{T}$ & 0.1628 \\
\hline 3 & rs7620350 & A & $\mathrm{T}$ & 0.2791 \\
\hline 3 & rs7620781 & A & G & 0.2907 \\
\hline 3 & rs138640574 & A & C & 0.2558 \\
\hline 3 & rs35740940 & G & C & 0.0697 \\
\hline 3 & rs77059190 & $\mathrm{T}$ & C & 0.2558 \\
\hline 3 & rs75448974 & A & G & 0.2442 \\
\hline 3 & rs73884819 & $\mathrm{T}$ & G & 0.2442 \\
\hline 3 & rs9290779 & A & C & 0.1628 \\
\hline 3 & rs145536424 & $\mathrm{T}$ & A & 0.0581 \\
\hline 3 & rs55831983 & C & $\mathrm{T}$ & 0.1047 \\
\hline 3 & rs1879259 & A & G & 0.2209 \\
\hline 3 & rs4148557 & A & G & 0.3256 \\
\hline 3 & rs7610724 & G & $\mathrm{T}$ & 0.0697 \\
\hline 3 & rs7613237 & $\mathrm{C}$ & $\mathrm{T}$ & 0.407 \\
\hline 3 & rs939335 & A & G & 0.2791 \\
\hline 3 & rs11917442 & $\mathrm{T}$ & C & 0.0581 \\
\hline 3 & rs201188880 & CT & C & 0.2442 \\
\hline
\end{tabular}

Table 1-A1 continues in the next column $\rightarrow$
Table 1-A1 (Continues...): Minor allele frequencies for 1846 polymorphisms in 43 black South Africans.

\begin{tabular}{|c|c|c|c|c|}
\hline Chromosome & Polymorphism & Minor allele & Major allele & $\begin{array}{l}\text { Minor allele } \\
\text { frequency }\end{array}$ \\
\hline 3 & rs11711219 & C & $A$ & 0.2558 \\
\hline 3 & rs10937159 & $A$ & C & 0.2558 \\
\hline 3 & rs7622660 & C & $T$ & 0.0930 \\
\hline 3 & rs36092077 & $A$ & G & 0.1628 \\
\hline 3 & rs141952918 & G & $A$ & 0.0581 \\
\hline 3 & rs6792482 & $\mathrm{T}$ & C & 0.2442 \\
\hline 3 & rs6779545 & C & A & 0.2442 \\
\hline 3 & rs6443930 & G & C & 0.2442 \\
\hline 3 & rs111689452 & A & G & 0.0348 \\
\hline 3 & rs1000952 & G & $A$ & 0.1163 \\
\hline 3 & rs6765152 & $A$ & C & 0.0814 \\
\hline 3 & rs56006127 & A & G & 0.0465 \\
\hline 3 & rs7621975 & G & $A$ & 0.4186 \\
\hline 3 & rs56060490 & C & $A$ & 0.0465 \\
\hline 3 & rs55741914 & G & A & 0.0814 \\
\hline 3 & rs57484150 & $\mathrm{T}$ & C & 0.0814 \\
\hline 3 & rs10937161 & $\mathrm{T}$ & C & 0.1977 \\
\hline 3 & rs58413046 & C & $\mathrm{T}$ & 0.0697 \\
\hline 3 & rs60791004 & A & G & 0.1628 \\
\hline 3 & rs7430671 & C & G & 0.3605 \\
\hline 3 & rs73183426 & G & C & 0.1977 \\
\hline 3 & rs74763842 & $\mathrm{T}$ & G & 0.4186 \\
\hline 3 & rs116348145 & $T$ & C & 0.0814 \\
\hline 3 & rs56889675 & $\mathrm{T}$ & G & 0.2558 \\
\hline 3 & rs10470524 & $\mathrm{T}$ & G & 0.2209 \\
\hline 3 & rs7641834 & $T$ & C & 0.1977 \\
\hline 3 & rs115021567 & G & A & 0.1977 \\
\hline 3 & rs60179621 & A & G & 0.1977 \\
\hline 3 & rs6762938 & $\mathrm{T}$ & C & 0.314 \\
\hline 3 & rs6794223 & G & A & 0.1395 \\
\hline 3 & rs7616916 & A & C & 0.0814 \\
\hline 3 & rs75574047 & C & A & 0.0814 \\
\hline 3 & rs6807271 & $A$ & G & 0.314 \\
\hline 3 & rs6807670 & A & G & 0.3953 \\
\hline 3 & rs13094369 & A & G & 0.3953 \\
\hline 3 & rs7610344 & $\mathrm{T}$ & G & 0.2791 \\
\hline 3 & rs7427051 & A & G & 0.2442 \\
\hline 3 & rs28476779 & G & A & 0.2791 \\
\hline 3 & rs9851097 & A & G & 0.2791 \\
\hline 3 & rs11924955 & $\mathrm{T}$ & C & 0.2558 \\
\hline 3 & rs60964651 & AT & A & 0.2791 \\
\hline 3 & rs79813226 & A & C & 0.2442 \\
\hline 3 & rs6779862 & A & C & 0.2442 \\
\hline 3 & rs6443933 & G & $A$ & 0.3605 \\
\hline 3 & rs13320474 & $A$ & G & 0.2791 \\
\hline 4 & rs2725215 & $\mathrm{T}$ & C & 0.1395 \\
\hline 4 & rs2728108 & $A$ & C & 0.0814 \\
\hline 4 & rs111917717 & $\mathrm{T}$ & $A$ & 0.0465 \\
\hline 4 & rs17013754 & G & $A$ & 0.1163 \\
\hline 4 & rs2728107 & $\mathrm{T}$ & C & 0.0465 \\
\hline 4 & rs17013761 & $\mathrm{T}$ & C & 0.1163 \\
\hline 4 & rs6843032 & $\mathrm{T}$ & G & 0.4767 \\
\hline 4 & rs113152807 & $T$ & C & 0.0465 \\
\hline 4 & rs7672253 & A & G & 0.0581 \\
\hline 4 & rs116685328 & $\mathrm{T}$ & C & 0.0465 \\
\hline 4 & rs4336187 & A & G & 0.2791 \\
\hline 4 & rs2728121 & $\mathrm{T}$ & C & 0.2093 \\
\hline 4 & rs10965 & A & G & 0.1395 \\
\hline 4 & rs74712548 & A & C & 0.1047 \\
\hline 4 & rs201502076_t3 & $\mathrm{T}$ & $\mathrm{C}$ & 0.4767 \\
\hline
\end{tabular}

Table 1-A1 continues on the next page $\rightarrow$ 
Table 1-A1 (Continues...): Minor allele frequencies for 1846 polymorphisms in 43 black South Africans.

\begin{tabular}{|c|c|c|c|c|}
\hline Chromosome & Polymorphism & Minor allele & Major allele & $\begin{array}{l}\text { Minor allele } \\
\text { frequency }\end{array}$ \\
\hline 4 & rs 45542445 & A & $T$ & 0.0581 \\
\hline 4 & rs115770495 & $\mathrm{T}$ & C & 0.0581 \\
\hline 4 & rs1448784 & G & A & 0.0465 \\
\hline 4 & rs4148159 & $\mathrm{T}$ & A & 0.1744 \\
\hline 4 & rs2231164 & $\mathrm{T}$ & C & 0.2907 \\
\hline 4 & rs2725267 & A & G & 0.3023 \\
\hline 4 & rs2231162 & A & G & 0.314 \\
\hline 4 & rs2231159 & C & A & 0.2093 \\
\hline 4 & rs2231158 & C & $\mathrm{T}$ & 0.2093 \\
\hline 4 & rs45621036 & C & $\mathrm{T}$ & 0.1628 \\
\hline 4 & rs 45556834 & G & $A$ & 0.2093 \\
\hline 4 & rs45469894 & C & $\mathrm{T}$ & 0.1628 \\
\hline 4 & rs1383586 & G & A & 0.3023 \\
\hline 4 & rs1383584 & A & G & 0.3023 \\
\hline 4 & rs 45592333 & C & $\mathrm{T}$ & 0.1628 \\
\hline 4 & rs10433946 & C & $\mathrm{T}$ & 0.0581 \\
\hline 4 & rs2231155 & $T$ & C & 0.1744 \\
\hline 4 & rs45566934 & $\mathrm{T}$ & C & 0.1628 \\
\hline 4 & rs141097556 & C & G & 0.1628 \\
\hline 4 & rs2622614 & $\mathrm{T}$ & C & 0.4651 \\
\hline 4 & rs2622613 & A & G & 0.314 \\
\hline 4 & rs45443398 & $\mathrm{T}$ & C & 0.1163 \\
\hline 4 & rs2231153 & $\mathrm{T}$ & C & 0.3023 \\
\hline 4 & rs141518597 & $\mathrm{T}$ & G & 0.0814 \\
\hline 4 & rs201742138 & AG & A & 0.1163 \\
\hline 4 & rs 28665233 & $A$ & G & 0.1395 \\
\hline 4 & rs2725264 & $\mathrm{T}$ & C & 0.1395 \\
\hline 4 & rs2725263 & C & $A$ & 0.1512 \\
\hline 4 & rs 2622628 & A & C & 0.3256 \\
\hline 4 & rs 12505410 & G & $\mathrm{T}$ & 0.0814 \\
\hline 4 & rs2622621 & G & C & 0.0581 \\
\hline 4 & rs13120400 & C & $\mathrm{T}$ & 0.0697 \\
\hline 4 & rs201460174 & G & GTCTCTCTCTстстстGTC & 0.0814 \\
\hline 4 & rs199994188 & C & СтстстстстстстG & 0.0814 \\
\hline 4 & rs57892861 & C & $T$ & 0.314 \\
\hline 4 & rs6532048 & G & A & 0.0814 \\
\hline 4 & rs2231147 & C & $\mathrm{T}$ & 0.1395 \\
\hline 4 & rs1871744 & C & $\mathrm{T}$ & 0.0814 \\
\hline 4 & rs2622618 & $A$ & G & 0.0465 \\
\hline 4 & rs2231144 & C & $\mathrm{T}$ & 0.3372 \\
\hline 4 & rs 113752350 & C & $\mathrm{T}$ & 0.3372 \\
\hline 4 & rs185151667 & $T$ & C & 0.3372 \\
\hline 4 & rs2725259 & $T$ & C & 0.0465 \\
\hline 4 & rs6832558 & $\mathrm{T}$ & C & 0.0465 \\
\hline 4 & rs2725258 & $T$ & C & 0.0465 \\
\hline 4 & rs2725256 & G & A & 0.3372 \\
\hline 4 & rs 45488400 & C & $\mathrm{T}$ & 0.3372 \\
\hline 4 & rs17013859 & $T$ & C & 0.3372 \\
\hline 4 & rs200576598 & $A G$ & A & 0.0581 \\
\hline 4 & rs2725255 & A & G & 0.0581 \\
\hline 4 & rs2622619 & G & C & 0.0465 \\
\hline 4 & rs17013870 & C & $\mathrm{T}$ & 0.3372 \\
\hline 4 & rs72875335 & A & G & 0.3372 \\
\hline 4 & rs113737399 & A & G & 0.314 \\
\hline 4 & rs2622631 & G & A & 0.0581 \\
\hline 4 & rs2622632 & G & A & 0.0465 \\
\hline 4 & rs 12641369 & A & G & 0.4651 \\
\hline 4 & rs2725253 & C & $\mathrm{T}$ & 0.0581 \\
\hline 4 & rs2622617 & G & A & 0.0581 \\
\hline 4 & rs1564481 & $\mathrm{T}$ & $\mathrm{C}$ & 0.1628 \\
\hline
\end{tabular}

Table 1-A1 continues in the next column $\rightarrow$
Table 1-A1 (Continues...): Minor allele frequencies for 1846 polymorphisms in 43 black South Africans.

\begin{tabular}{|c|c|c|c|c|}
\hline Chromosome & Polymorphism & Minor allele & Major allele & $\begin{array}{l}\text { Minor allele } \\
\text { frequency }\end{array}$ \\
\hline 4 & rs 2725252 & A & C & 0.2907 \\
\hline 4 & rs13130891 & A & G & 0.5 \\
\hline 4 & rs4148149 & $\mathrm{T}$ & G & 0.4651 \\
\hline 4 & rs13137622 & G & $\mathrm{T}$ & 0.5 \\
\hline 4 & rs2046134 & A & G & 0.4186 \\
\hline 4 & rs2725250 & G & A & 0.2326 \\
\hline 4 & rs3114018 & C & A & 0.4651 \\
\hline 4 & rs3109823 & $\mathrm{T}$ & C & 0.5 \\
\hline 4 & rs17013881 & $\mathrm{T}$ & C & 0.1163 \\
\hline 4 & rs113094792 & $\mathrm{T}$ & C & 0.0465 \\
\hline 4 & rs 2622627 & $A$ & C & 0.4419 \\
\hline 4 & rs73844307 & C & $\mathrm{T}$ & 0.0465 \\
\hline 4 & rs2725249 & C & A & 0.4419 \\
\hline 4 & rs6857600 & $\mathrm{T}$ & C & 0.3605 \\
\hline 4 & rs115055824 & G & A & 0.0581 \\
\hline 4 & rs114085125 & A & C & 0.0581 \\
\hline 4 & rs114726329 & $\mathrm{T}$ & C & 0.0581 \\
\hline 4 & rs11934545 & A & G & 0.0581 \\
\hline 4 & rs 2622626 & A & C & 0.4419 \\
\hline 4 & rs11287117 & $\mathrm{T}$ & TG & 0.4535 \\
\hline 4 & rs11413103 & GT & G & 0.4419 \\
\hline 4 & rs6532049 & $\mathrm{T}$ & C & 0.4767 \\
\hline 4 & rs2725247 & A & G & 0.2326 \\
\hline 4 & rs17731799 & $\mathrm{T}$ & G & 0.3605 \\
\hline 4 & rs2725246 & A & G & 0.2326 \\
\hline 4 & rs2725245 & A & G & 0.2326 \\
\hline 4 & rs2725244 & C & $\mathrm{T}$ & 0.4651 \\
\hline 4 & rs2622624 & C & $\mathrm{T}$ & 0.2326 \\
\hline 4 & rs 2725242 & A & $\mathrm{T}$ & 0.4651 \\
\hline 4 & rs1466480 & A & G & 0.3488 \\
\hline 4 & rs6820121 & C & $\mathrm{T}$ & 0.0581 \\
\hline 4 & rs6843273 & A & G & 0.0581 \\
\hline 4 & rs6843542 & $\mathrm{T}$ & C & 0.0697 \\
\hline 4 & rs6844086 & A & G & 0.0581 \\
\hline 4 & rs2622604 & $\mathrm{T}$ & C & 0.1163 \\
\hline 4 & rs111766106 & A & G & 0.1279 \\
\hline 4 & rs45604438 & $\mathrm{T}$ & G & 0.0814 \\
\hline 4 & rs3114019 & C & $\mathrm{T}$ & 0.0697 \\
\hline 4 & rs3114020 & C & $\mathrm{T}$ & 0.1744 \\
\hline 4 & rs11732936 & G & A & 0.1628 \\
\hline 4 & rs10011796 & $\mathrm{T}$ & C & 0.314 \\
\hline 4 & rs28440048 & $\mathrm{T}$ & C & 0.1163 \\
\hline 4 & rs35228269 & G & A & 0.3488 \\
\hline 4 & rs7657441 & C & $\mathrm{T}$ & 0.1744 \\
\hline 4 & rs6837857 & A & C & 0.3488 \\
\hline 4 & rs35839768 & G & A & 0.3488 \\
\hline 4 & rs35537015 & C & $\mathrm{T}$ & 0.3488 \\
\hline 4 & rs34731996 & C & $\mathrm{T}$ & 0.3488 \\
\hline 4 & rs35229708 & C & $\mathrm{T}$ & 0.3488 \\
\hline 4 & rs35252139 & $\mathrm{T}$ & C & 0.3488 \\
\hline 4 & rs7699188 & A & G & 0.3488 \\
\hline 4 & rs7699279 & $\mathrm{T}$ & C & 0.3488 \\
\hline 4 & rs7657531 & $C$ & A & 0.3488 \\
\hline 4 & rs7682521 & G & $\mathrm{T}$ & 0.3488 \\
\hline 4 & rs7658211 & A & G & 0.3488 \\
\hline 4 & rs7657757 & G & A & 0.3488 \\
\hline 4 & rs7682757 & $C$ & $\mathrm{~T}$ & 0.3488 \\
\hline 4 & rs7657928 & $C$ & A & 0.3488 \\
\hline 4 & rs7658584 & A & G & 0.3488 \\
\hline 4 & rs112385917 & CTTTA & C & 0.3488 \\
\hline
\end{tabular}

Table 1-A1 continues on the next page $\rightarrow$ 
Table 1-A1 (Continues...): Minor allele frequencies for 1846 polymorphisms in 43 black South Africans.

\begin{tabular}{|c|c|c|c|c|}
\hline Chromosome & Polymorphism & Minor allele & Major allele & $\begin{array}{l}\text { Minor allele } \\
\text { frequency }\end{array}$ \\
\hline 4 & rs13128241 & $\mathrm{T}$ & $\mathrm{C}$ & 0.3488 \\
\hline 4 & rs 13128083 & G & A & 0.3488 \\
\hline 4 & rs55987521 & $\mathrm{T}$ & A & 0.3721 \\
\hline 4 & rs60816576 & $\mathrm{T}$ & G & 0.3953 \\
\hline 4 & rs57454797 & C & $\mathrm{T}$ & 0.3953 \\
\hline 4 & rs1481014 & A & C & 0.3721 \\
\hline 4 & rs13135956 & A & G & 0.3721 \\
\hline 4 & rs76462878 & $\mathrm{T}$ & A & 0.0814 \\
\hline 4 & rs6821227 & C & $\mathrm{T}$ & 0.2442 \\
\hline 4 & rs6821239 & A & G & 0.2442 \\
\hline 4 & rs9784454 & C & $\mathrm{T}$ & 0.2442 \\
\hline 4 & rs6532053 & A & G & 0.2442 \\
\hline 4 & rs6833713 & G & A & 0.2442 \\
\hline 4 & rs6833950 & G & A & 0.2442 \\
\hline 4 & rs2127861 & C & G & 0.2442 \\
\hline 4 & rs2127863 & $\mathrm{T}$ & C & 0.2442 \\
\hline 4 & rs70959608 & C & CA & 0.2442 \\
\hline 4 & rs6854688 & G & A & 0.314 \\
\hline 4 & rs11097182 & $\mathrm{T}$ & C & 0.3837 \\
\hline 4 & rs113611770 & C & $A$ & 0.0465 \\
\hline 4 & rs112710034 & G & C & 0.0465 \\
\hline 4 & rs6532055 & C & $\mathrm{T}$ & 0.1628 \\
\hline 4 & rs10856870 & C & $\mathrm{T}$ & 0.4651 \\
\hline 4 & rs150614746 & G & A & 0.0465 \\
\hline 4 & rs 140027200 & $\mathrm{~T}$ & C & 0.0465 \\
\hline 4 & rs75048878 & $\mathrm{T}$ & G & 0.1279 \\
\hline 4 & rs4693930 & A & G & 0.1047 \\
\hline 4 & rs139884402 & G & C & 0.0465 \\
\hline 4 & rs11723264 & A & G & 0.3953 \\
\hline 7 & rs67721532 & G & A & 0.1512 \\
\hline 7 & rs 45505292 & C & $\mathrm{T}$ & 0.0930 \\
\hline 7 & rs112113287 & G & GTCGTGTTT & 0.407 \\
\hline 7 & rs60123540 & C & $\mathrm{T}$ & 0.407 \\
\hline 7 & rs17149637 & A & G & 0.407 \\
\hline 7 & rs 17149640 & C & A & 0.407 \\
\hline 7 & rs17149641 & C & $\mathrm{T}$ & 0.407 \\
\hline 7 & rs 45580239 & A & G & 0.2093 \\
\hline 7 & rs 45546132 & $\mathrm{~T}$ & C & 0.407 \\
\hline 7 & rs45447097 & A & G & 0.407 \\
\hline 7 & rs17149647 & C & $\mathrm{T}$ & 0.3837 \\
\hline 7 & rs 45607141 & G & A & 0.407 \\
\hline 7 & rs17149652 & $\mathrm{T}$ & G & 0.407 \\
\hline 7 & rs4148817 & G & C & 0.407 \\
\hline 7 & rs4148815 & $\mathrm{T}$ & A & 0.407 \\
\hline 7 & rs66463970 & GT & G & 0.407 \\
\hline 7 & rs45593435 & G & A & 0.407 \\
\hline 7 & rs17149660 & C & $\mathrm{T}$ & 0.407 \\
\hline 7 & rs 45502492 & $\mathrm{~T}$ & C & 0.3605 \\
\hline 7 & rs 45526438 & A & G & 0.3605 \\
\hline 7 & rs 45605032 & $\mathrm{~T}$ & C & 0.407 \\
\hline 7 & rs 45590633 & $\mathrm{~T}$ & C & 0.1512 \\
\hline 7 & rs 45446703 & A & G & 0.0930 \\
\hline 7 & rs4148814 & C & $\mathrm{T}$ & 0.3605 \\
\hline 7 & rs2302385 & C & $\mathrm{T}$ & 0.3605 \\
\hline 7 & rs2302386 & G & A & 0.3605 \\
\hline 7 & rs2302387 & A & G & 0.407 \\
\hline 7 & rs7782643 & A & G & 0.1512 \\
\hline 7 & rs 45564638 & G & A & 0.0465 \\
\hline 7 & rs2888611 & G & C & 0.407 \\
\hline 7 & rs4148808 & $\mathrm{C}$ & $\mathrm{T}$ & 0.2674 \\
\hline
\end{tabular}

Table 1-A1 continues in the next column $\rightarrow$
Table 1-A1 (Continues...): Minor allele frequencies for 1846 polymorphisms in 43 black South Africans.

\begin{tabular}{|c|c|c|c|c|}
\hline Chromosome & Polymorphism & Minor allele & Major allele & $\begin{array}{l}\text { Minor allele } \\
\text { frequency }\end{array}$ \\
\hline 7 & rs4148807 & A & G & 0.2674 \\
\hline 7 & rs4148805 & A & G & 0.2674 \\
\hline 7 & rs10556862 & A & ATG & 0.2209 \\
\hline 7 & rs73399961 & G & A & 0.0581 \\
\hline 7 & rs45555137 & $\mathrm{T}$ & TTC & 0.2209 \\
\hline 7 & rs45443201 & C & $\mathrm{T}$ & 0.0814 \\
\hline 7 & rs12673662 & G & C & 0.2209 \\
\hline 7 & rs66510518 & G & C & 0.2791 \\
\hline 7 & rs73705216 & G & $\mathrm{T}$ & 0.0814 \\
\hline 7 & rs6465116 & A & G & 0.2326 \\
\hline 7 & rs148890468 & $A$ & C & 0.2326 \\
\hline 7 & rs11977881 & C & $\mathrm{T}$ & 0.2558 \\
\hline 7 & rs59934297 & G & $\mathrm{T}$ & 0.2791 \\
\hline 7 & rs58083477 & C & $\mathrm{T}$ & 0.2791 \\
\hline 7 & rs59616301 & C & $\mathrm{T}$ & 0.2791 \\
\hline 7 & rs7805184 & A & G & 0.2791 \\
\hline 7 & rs66869391 & A & G & 0.2791 \\
\hline 7 & rs17209662 & A & G & 0.2791 \\
\hline 7 & rs2178658 & $\mathrm{T}$ & G & 0.2093 \\
\hline 7 & rs17275514 & G & A & 0.2791 \\
\hline 7 & rs78551545 & A & G & 0.0581 \\
\hline 7 & rs998671 & $\mathrm{T}$ & C & 0.2674 \\
\hline 7 & rs12538707 & C & $\mathrm{T}$ & 0.2326 \\
\hline 7 & rs17209837 & C & $\mathrm{T}$ & 0.2791 \\
\hline 7 & rs150974072 & A & AAT & 0.2558 \\
\hline 7 & rs12672720 & G & A & 0.2326 \\
\hline 7 & rs60541816 & A & G & 0.2326 \\
\hline 7 & rs60916103 & G & A & 0.2326 \\
\hline 7 & rs6946119 & C & $\mathrm{T}$ & 0.0581 \\
\hline 7 & rs73198349 & A & C & 0.1163 \\
\hline 7 & rs7802555 & C & A & 0.2326 \\
\hline 7 & rs7802783 & $\mathrm{T}$ & C & 0.2326 \\
\hline 7 & rs3842 & C & $\mathrm{T}$ & 0.2442 \\
\hline 7 & rs28364278_t3 & 1 & D & 0.0465 \\
\hline 7 & rs28364277 & T & C & 0.1279 \\
\hline 7 & rs17064 & A & $\mathrm{T}$ & 0.2326 \\
\hline 7 & rs28401816 & C & $\mathrm{T}$ & 0.1628 \\
\hline 7 & rs28401814 & A & G & 0.1628 \\
\hline 7 & rs1882478 & C & $\mathrm{T}$ & 0.3837 \\
\hline 7 & rs2235048 & G & A & 0.0930 \\
\hline 7 & rs2235047 & C & A & 0.2093 \\
\hline 7 & rs1045642 & A & G & 0.0930 \\
\hline 7 & rs2229107 & $\mathrm{T}$ & A & 0.1047 \\
\hline 7 & rs10808071 & G & A & 0.2326 \\
\hline 7 & rs1002204 & A & C & 0.1395 \\
\hline 7 & rs17149699 & $\mathrm{T}$ & C & 0.3023 \\
\hline 7 & rs112292979 & A & G & 0.1047 \\
\hline 7 & rs4148751 & C & $\mathrm{T}$ & 0.1628 \\
\hline 7 & rs4148749 & C & G & 0.0581 \\
\hline 7 & rs111526989 & $\mathrm{T}$ & G & 0.0697 \\
\hline 7 & rs28401796 & A & G & 0.1744 \\
\hline 7 & rs112845881 & C & $\mathrm{T}$ & 0.0697 \\
\hline 7 & rs1922244 & A & G & 0.2674 \\
\hline 7 & rs113724043 & $\mathrm{T}$ & C & 0.0697 \\
\hline 7 & rs187721980 & A & G & 0.0697 \\
\hline 7 & rs3028303 & TTC & $\mathrm{T}$ & 0.2326 \\
\hline 7 & rs28401792 & G & GATCCACTATGCCTA & 0.1744 \\
\hline 7 & rs4148747 & A & $\mathrm{T}$ & 0.1977 \\
\hline 7 & rs4148746 & G & GT & 0.1977 \\
\hline 7 & rs4148745 & $\mathrm{T}$ & G & 0.1395 \\
\hline
\end{tabular}

Table 1-A1 continues on the next page $\rightarrow$ 
Table 1-A1 (Continues...): Minor allele frequencies for 1846 polymorphisms in 43 black South Africans.

\begin{tabular}{|c|c|c|c|c|}
\hline Chromosome & Polymorphism & Minor allele & Major allele & $\begin{array}{l}\text { Minor allele } \\
\text { frequency }\end{array}$ \\
\hline 7 & rs 28401781 & $\mathrm{~T}$ & C & 0.2442 \\
\hline 7 & rs147600670 & A & $A C$ & 0.1744 \\
\hline 7 & rs2235067 & $\mathrm{T}$ & C & 0.1279 \\
\hline 7 & rs4148743 & $\mathrm{T}$ & C & 0.314 \\
\hline 7 & rs1882477 & C & G & 0.2326 \\
\hline 7 & rs2373589 & $\mathrm{T}$ & C & 0.3488 \\
\hline 7 & rs113822506 & A & G & 0.1047 \\
\hline 7 & rs4148740 & G & A & 0.186 \\
\hline 7 & rs147898841 & C & $\mathrm{T}$ & 0.0697 \\
\hline 7 & rs113521552 & $\mathrm{C}$ & $\mathrm{T}$ & 0.0697 \\
\hline 7 & rs10246696 & A & G & 0.186 \\
\hline 7 & rs11979702 & A & $\mathrm{T}$ & 0.1279 \\
\hline 7 & rs2141849 & A & C & 0.1628 \\
\hline 7 & rs112216837 & G & C & 0.0697 \\
\hline 7 & rs55912869 & C & $\mathrm{T}$ & 0.186 \\
\hline 7 & rs2373588 & A & G & 0.1628 \\
\hline 7 & rs10280101 & C & A & 0.186 \\
\hline 7 & rs35572298 & A & AG & 0.0814 \\
\hline 7 & rs150867018 & G & A & 0.0697 \\
\hline 7 & rs16885829 & C & $\mathrm{T}$ & 0.1395 \\
\hline 7 & rs67151359 & G & GT & 0.1628 \\
\hline 7 & rs10225473 & G & A & 0.1279 \\
\hline 7 & rs6971264 & A & G & 0.1395 \\
\hline 7 & rs35280822 & A & G & 0.0814 \\
\hline 7 & rs10240953 & $\mathrm{T}$ & G & 0.0697 \\
\hline 7 & rs7787082 & G & A & 0.2442 \\
\hline 7 & rs2373587 & G & C & 0.1628 \\
\hline 7 & rs113158842 & G & $\mathrm{T}$ & 0.0697 \\
\hline 7 & rs 28681479 & C & $\mathrm{T}$ & 0.2093 \\
\hline 7 & rs2373585 & $\mathrm{T}$ & C & 0.1628 \\
\hline 7 & rs2032583 & G & A & 0.186 \\
\hline 7 & rs4148739 & C & $\mathrm{T}$ & 0.186 \\
\hline 7 & rs11983225 & C & $\mathrm{T}$ & 0.186 \\
\hline 7 & rs113106026 & G & A & 0.0697 \\
\hline 7 & rs 10236274 & G & A & 0.2442 \\
\hline 7 & rs183410334 & G & A & 0.0697 \\
\hline 7 & rs11760837 & C & $\mathrm{T}$ & 0.186 \\
\hline 7 & rs139774375 & A & G & 0.0697 \\
\hline 7 & rs 11972405 & C & $\mathrm{T}$ & 0.186 \\
\hline 7 & rs10274587 & A & G & 0.1512 \\
\hline 7 & rs28381959 & A & G & 0.1047 \\
\hline 7 & rs 28381958 & G & GA & 0.3721 \\
\hline 7 & rs 10248420 & A & G & 0.3721 \\
\hline 7 & rs149043325 & A & G & 0.0581 \\
\hline 7 & rs113764224 & G & A & 0.0814 \\
\hline 7 & rs2235040 & $\mathrm{T}$ & C & 0.1512 \\
\hline 7 & rs111992902 & $\mathrm{T}$ & C & 0.0697 \\
\hline 7 & rs79890058 & A & G & 0.0348 \\
\hline 7 & rs28381951 & $\mathrm{T}$ & G & 0.0348 \\
\hline 7 & rs12668877 & $\mathrm{T}$ & C & 0.2326 \\
\hline 7 & rs111538144 & G & A & 0.0697 \\
\hline 7 & rs3789246 & $\mathrm{T}$ & C & 0.2326 \\
\hline 7 & rs2235064 & G & $\mathrm{T}$ & 0.0697 \\
\hline 7 & rs7795817 & $\mathrm{T}$ & C & 0.2326 \\
\hline 7 & rs12154941 & $\mathrm{T}$ & C & 0.0814 \\
\hline 7 & rs28381940 & G & A & 0.1744 \\
\hline 7 & rs4148737 & $\mathrm{C}$ & $\mathrm{T}$ & 0.4651 \\
\hline 7 & rs4148736 & A & G & 0.4651 \\
\hline 7 & rs4728700 & $\mathrm{T}$ & C & 0.1977 \\
\hline 7 & rs28381933 & G & A & 0.0465 \\
\hline
\end{tabular}

Table 1-A1 continues in the next column $\rightarrow$
Table 1-A1 (Continues...): Minor allele frequencies for 1846 polymorphisms in 43 black South Africans.

\begin{tabular}{|c|c|c|c|c|}
\hline Chromosome & Polymorphism & Minor allele & Major allele & $\begin{array}{l}\text { Minor allele } \\
\text { frequency }\end{array}$ \\
\hline 7 & rs6961419 & C & $\mathrm{T}$ & 0.4651 \\
\hline 7 & rs6961882 & C & $\mathrm{T}$ & 0.4651 \\
\hline 7 & rs60503369 & A & $\mathrm{T}$ & 0.0465 \\
\hline 7 & rs6980101 & $\mathrm{T}$ & C & 0.2674 \\
\hline 7 & rs4148735 & $\mathrm{T}$ & C & 0.4651 \\
\hline 7 & rs1922242 & $\mathrm{T}$ & A & 0.4651 \\
\hline 7 & rs2235046 & $T$ & C & 0.1628 \\
\hline 7 & rs2091766 & $\mathrm{T}$ & C & 0.4651 \\
\hline 7 & rs 12704363 & $\mathrm{~T}$ & C & 0.2093 \\
\hline 7 & rs28381909 & A & G & 0.2907 \\
\hline 7 & rs112489538 & $\mathrm{T}$ & A & 0.0697 \\
\hline 7 & rs10713802 & A & AG & 0.2907 \\
\hline 7 & rs115780656 & $\mathrm{T}$ & $\mathrm{C}$ & 0.1163 \\
\hline 7 & rs60412009 & C & $\mathrm{T}$ & 0.1279 \\
\hline 7 & rs113686230 & A & G & 0.1163 \\
\hline 7 & rs143422171 & A & G & 0.0465 \\
\hline 7 & rs2235013 & $\mathrm{T}$ & C & 0.4186 \\
\hline 7 & rs28381898 & $\mathrm{T}$ & C & 0.0465 \\
\hline 7 & rs2235035 & $A$ & G & 0.1628 \\
\hline 7 & rs2235033 & G & $A$ & 0.4186 \\
\hline 7 & rs1128503 & $A$ & G & 0.0930 \\
\hline 7 & rs2032585 & C & $\mathrm{T}$ & 0.0465 \\
\hline 7 & rs2235030 & $A$ & G & 0.0697 \\
\hline 7 & rs10276036 & C & $\mathrm{T}$ & 0.1628 \\
\hline 7 & rs113125275 & G & A & 0.1163 \\
\hline 7 & rs4728702 & $A$ & $\mathrm{~T}$ & 0.0930 \\
\hline 7 & rs28381893 & C & $T$ & 0.0465 \\
\hline 7 & rs12704364 & $\mathrm{T}$ & C & 0.4186 \\
\hline 7 & rs56735241 & C & $T$ & 0.0930 \\
\hline 7 & rs6961665 & $A$ & C & 0.4186 \\
\hline 7 & rs3789244 & G & $T$ & 0.1628 \\
\hline 7 & rs2235028 & A & G & 0.0465 \\
\hline 7 & rs2235027 & $\mathrm{T}$ & G & 0.4186 \\
\hline 7 & rs2235026 & $\mathrm{T}$ & C & 0.1628 \\
\hline 7 & rs1922240 & C & $T$ & 0.1628 \\
\hline 7 & rs139722542 & A & G & 0.0930 \\
\hline 7 & rs114106519 & C & $\mathrm{T}$ & 0.0930 \\
\hline 7 & rs199812223 & AT & A & 0.0465 \\
\hline 7 & rs1922241 & A & G & 0.1628 \\
\hline 7 & rs57924923 & A & G & 0.4186 \\
\hline 7 & rs11772987 & G & C & 0.1279 \\
\hline 7 & rs60749469 & C & $\mathrm{T}$ & 0.4186 \\
\hline 7 & rs10244266 & G & $\mathrm{T}$ & 0.1279 \\
\hline 7 & rs1882479 & G & A & 0.1163 \\
\hline 7 & rs2235023 & $T$ & C & 0.4186 \\
\hline 7 & rs28381866 & G & A & 0.0930 \\
\hline 7 & rs10274441 & G & A & 0.1279 \\
\hline 7 & rs11975994 & G & A & 0.0930 \\
\hline 7 & rs28381853 & A & G & 0.0465 \\
\hline 7 & rs28381851 & G & $\mathrm{T}$ & 0.0465 \\
\hline 7 & rs112858219 & A & G & 0.0697 \\
\hline 7 & rs28381848 & C & $\mathrm{T}$ & 0.0814 \\
\hline 7 & rs58525782 & C & A & 0.0465 \\
\hline 7 & rs1024409 & A & G & 0.0697 \\
\hline 7 & rs201620488_t3 & G & $T$ & 0.3605 \\
\hline 7 & rs1989830 & A & G & 0.3023 \\
\hline 7 & rs1989829 & $T$ & C & 0.3023 \\
\hline 7 & rs73387255 & A & G & 0.2093 \\
\hline 7 & rs7809208 & A & C & 0.3953 \\
\hline 7 & rs2188526 & $\mathrm{T}$ & $\mathrm{C}$ & 0.0465 \\
\hline
\end{tabular}

Table 1-A1 continues on the next page $\rightarrow$ 
Table 1-A1 (Continues...): Minor allele frequencies for 1846 polymorphisms in 43 black South Africans.

\begin{tabular}{|c|c|c|c|c|}
\hline Chromosome & Polymorphism & Minor allele & Major allele & $\begin{array}{l}\text { Minor allele } \\
\text { frequency }\end{array}$ \\
\hline 7 & rs3789243 & G & A & 0.4651 \\
\hline 7 & rs1858923 & G & A & 0.0465 \\
\hline 7 & rs3214119 & $\mathrm{T}$ & TC & 0.0697 \\
\hline 7 & rs3213619 & G & A & 0.1395 \\
\hline 7 & rs 28381800 & A & $\mathrm{T}$ & 0.0697 \\
\hline 7 & rs11395081 & AT & A & 0.0697 \\
\hline 7 & rs4148731 & A & G & 0.0697 \\
\hline 7 & rs4148730 & G & A & 0.0697 \\
\hline 7 & rs 28381775 & C & $\mathrm{T}$ & 0.0697 \\
\hline 7 & rs28381772 & $\mathrm{T}$ & G & 0.0697 \\
\hline 7 & rs11975403 & A & G & 0.0581 \\
\hline 7 & rs10231033 & G & A & 0.0697 \\
\hline 7 & rs4148729 & G & $\mathrm{T}$ & 0.0697 \\
\hline 7 & rs17149840 & A & G & 0.0697 \\
\hline 7 & rs75974753 & C & $\mathrm{T}$ & 0.0697 \\
\hline 7 & rs10280686 & $\mathrm{T}$ & A & 0.0697 \\
\hline 7 & rs10233247 & G & A & 0.0814 \\
\hline 7 & rs10224594 & C & $\mathrm{T}$ & 0.0697 \\
\hline 7 & rs 10275831 & $\mathrm{~T}$ & C & 0.0697 \\
\hline 7 & rs10246878 & A & G & 0.0581 \\
\hline 7 & rs200339290 & C & CT & 0.0930 \\
\hline 7 & rs10267099 & G & A & 0.1163 \\
\hline 7 & rs76348194 & A & G & 0.0930 \\
\hline 7 & rs11973812 & G & C & 0.0697 \\
\hline 7 & rs11977492 & A & $\mathrm{T}$ & 0.0697 \\
\hline 7 & rs 28483333 & $\mathrm{~T}$ & C & 0.0697 \\
\hline 7 & rs7810499 & C & $\mathrm{T}$ & 0.0697 \\
\hline 7 & rs 28746495 & $\mathrm{~T}$ & C & 0.1047 \\
\hline 7 & rs 28746492 & G & A & 0.0697 \\
\hline 7 & rs6951067 & $\mathrm{T}$ & $\mathrm{C}$ & 0.4884 \\
\hline 7 & rs142999199 & A & AG & 0.0697 \\
\hline 7 & rs78413330 & A & G & 0.0697 \\
\hline 7 & rs76190983 & A & G & 0.0697 \\
\hline 7 & rs6465117 & A & G & 0.1977 \\
\hline 7 & rs10254392 & C & $\mathrm{T}$ & 0.0697 \\
\hline 7 & rs2106522 & G & $\mathrm{T}$ & 0.1047 \\
\hline 7 & rs2157930 & A & G & 0.1163 \\
\hline 7 & rs58101885 & A & G & 0.0930 \\
\hline 7 & rs75910150 & $\mathrm{T}$ & C & 0.0697 \\
\hline 7 & rs73705296 & A & G & 0.0930 \\
\hline 7 & rs12540931 & $\mathrm{C}$ & $\mathrm{T}$ & 0.1977 \\
\hline 7 & rs73705298 & A & G & 0.0930 \\
\hline 7 & rs 145424538 & A & AAAC & 0.0697 \\
\hline 7 & rs77394523 & $\mathrm{T}$ & C & 0.0930 \\
\hline 7 & rs6957599 & A & G & 0.0697 \\
\hline 7 & rs11983274 & G & A & 0.0697 \\
\hline 7 & rs7796247 & A & G & 0.0697 \\
\hline 7 & rs1015415 & $\mathrm{T}$ & A & 0.2093 \\
\hline 7 & rs6465118 & A & G & 0.1977 \\
\hline 7 & rs10278483 & $\mathrm{C}$ & $\mathrm{T}$ & 0.0697 \\
\hline 7 & rs2188530 & G & A & 0.0697 \\
\hline 7 & rs2188529 & A & $\mathrm{T}$ & 0.0697 \\
\hline 7 & rs201768076 & A & AT & 0.0930 \\
\hline 7 & rs17149864 & G & A & 0.2209 \\
\hline 7 & rs78854352 & $\mathrm{T}$ & C & 0.0697 \\
\hline 7 & rs74754276 & $\mathrm{T}$ & C & 0.0697 \\
\hline 7 & rs11972683 & $\mathrm{T}$ & C & 0.0697 \\
\hline 7 & rs10232449 & A & G & 0.0697 \\
\hline 7 & rs10261685 & C & A & 0.0697 \\
\hline 7 & rs10248345 & G & $\mathrm{T}$ & 0.0697 \\
\hline
\end{tabular}

Table 1-A1 continues in the next column $\rightarrow$
Table 1-A1 (Continues...): Minor allele frequencies for 1846 polymorphisms in 43 black South Africans.

\begin{tabular}{|c|c|c|c|c|}
\hline Chromosome & Polymorphism & Minor allele & Major allele & $\begin{array}{l}\text { Minor allele } \\
\text { frequency }\end{array}$ \\
\hline 7 & rs199704465 & $\mathrm{T}$ & TTAA & 0.0697 \\
\hline 7 & rs199718071 & $\mathrm{T}$ & TTA & 0.0697 \\
\hline 7 & rs28572147 & $\mathrm{T}$ & A & 0.0697 \\
\hline 7 & rs146812933 & A & G & 0.0697 \\
\hline 7 & rs10281645 & G & A & 0.0697 \\
\hline 7 & rs28774398 & A & $\mathrm{T}$ & 0.1047 \\
\hline 7 & rs10486997 & A & G & 0.0697 \\
\hline 7 & rs12537294 & C & $\mathrm{T}$ & 0.0697 \\
\hline 7 & rs2188523 & A & G & 0.0697 \\
\hline 7 & rs28879532 & A & $\mathrm{T}$ & 0.0697 \\
\hline 7 & rs10259182 & $\mathrm{T}$ & C & 0.1047 \\
\hline 7 & rs10275625 & $\mathrm{T}$ & C & 0.0697 \\
\hline 7 & rs10237438 & A & G & 0.0697 \\
\hline 7 & rs78598021 & $\mathrm{T}$ & C & 0.0697 \\
\hline 7 & rs17149882 & A & G & 0.0697 \\
\hline 7 & rs10230766 & A & $\mathrm{T}$ & 0.0697 \\
\hline 7 & rs10280904 & C & $\mathrm{T}$ & 0.0697 \\
\hline 7 & rs35378698 & CT & C & 0.0581 \\
\hline 7 & rs188284544 & $\mathrm{T}$ & C & 0.0697 \\
\hline 7 & rs181050063 & G & A & 0.0697 \\
\hline 7 & rs28624877 & A & $\mathrm{T}$ & 0.0697 \\
\hline 7 & rs13437761 & $\mathrm{T}$ & C & 0.0697 \\
\hline 7 & rs13438341 & C & A & 0.0697 \\
\hline 7 & rs10276409 & $\mathrm{T}$ & G & 0.0697 \\
\hline 7 & rs12532279 & G & $\mathrm{T}$ & 0.1047 \\
\hline 7 & rs6962399 & $\mathrm{T}$ & C & 0.0697 \\
\hline 7 & rs13437893 & A & G & 0.0697 \\
\hline 7 & rs77994152 & G & A & 0.0697 \\
\hline 7 & rs35648408 & C & G & 0.0581 \\
\hline 7 & rs147934073 & $\mathrm{T}$ & TACTTATCCTATG & 0.0697 \\
\hline 11 & rs2712788 & $\mathrm{T}$ & A & 0.2326 \\
\hline 11 & rs2851076 & C & A & 0.2209 \\
\hline 11 & rs2851077 & G & A & 0.2209 \\
\hline 11 & rs949071 & $\mathrm{T}$ & C & 0.2209 \\
\hline 11 & rs80217529 & A & G & 0.1047 \\
\hline 11 & rs2851078 & G & A & 0.2209 \\
\hline 11 & rs7124613 & $\mathrm{T}$ & C & 0.1047 \\
\hline 11 & rs2851079 & C & $\mathrm{T}$ & 0.2674 \\
\hline 11 & rs 2851080 & C & $\mathrm{T}$ & 0.2209 \\
\hline 11 & rs 2851081 & A & G & 0.2209 \\
\hline 11 & rs 2851082 & A & $\mathrm{T}$ & 0.2209 \\
\hline 11 & rs77953909 & G & A & 0.1047 \\
\hline 11 & rs1944615 & A & C & 0.1163 \\
\hline 11 & rs4944977 & C & $\mathrm{T}$ & 0.1047 \\
\hline 11 & rs28806232 & $\mathrm{T}$ & C & 0.0930 \\
\hline 11 & rs11236332 & G & C & 0.1628 \\
\hline 11 & rs2510657 & G & A & 0.2674 \\
\hline 11 & rs 2513653 & A & C & 0.2674 \\
\hline 11 & rs148952117 & C & $\mathrm{T}$ & 0.1047 \\
\hline 11 & rs2851087 & A & G & 0.2674 \\
\hline 11 & rs 2513655 & C & $\mathrm{T}$ & 0.2674 \\
\hline 11 & rs2465291 & C & $\mathrm{T}$ & 0.2674 \\
\hline 11 & rs 2712823 & A & G & 0.2674 \\
\hline 11 & rs2851088 & C & $\mathrm{T}$ & 0.2674 \\
\hline 11 & rs17133681 & C & A & 0.1047 \\
\hline 11 & rs2712827 & G & A & 0.1163 \\
\hline 11 & rs2712779 & A & $\mathrm{T}$ & 0.2674 \\
\hline 11 & rs2712780 & A & G & 0.0581 \\
\hline 11 & rs4944981 & A & G & 0.1512 \\
\hline 11 & rs143837090 & A & G & 0.0581 \\
\hline
\end{tabular}

Table 1-A1 continues on the next page $\rightarrow$ 
Table 1-A1 (Continues...): Minor allele frequencies for 1846 polymorphisms in 43 black South Africans.

\begin{tabular}{|c|c|c|c|c|}
\hline Chromosome & Polymorphism & Minor allele & Major allele & $\begin{array}{l}\text { Minor allele } \\
\text { frequency }\end{array}$ \\
\hline 11 & rs2513656 & $T$ & C & 0.2442 \\
\hline 11 & rs2712812 & $\mathrm{T}$ & C & 0.2442 \\
\hline 11 & rs2851091 & A & G & 0.3953 \\
\hline 11 & rs2712803 & $\mathrm{T}$ & C & 0.2442 \\
\hline 11 & rs2712791 & $A$ & G & 0.2442 \\
\hline 11 & rs2712794 & $A$ & G & 0.4535 \\
\hline 11 & rs12279394 & G & A & 0.4186 \\
\hline 11 & rs144636650 & $\mathrm{T}$ & C & 0.0930 \\
\hline 11 & rs2712799 & A & G & 0.1977 \\
\hline 11 & rs114000664 & $\mathrm{T}$ & C & 0.0930 \\
\hline 11 & rs79297525 & $T$ & C & 0.1395 \\
\hline 11 & rs11236348 & A & C & 0.407 \\
\hline 11 & rs 11236349 & G & $\mathrm{T}$ & 0.407 \\
\hline 11 & rs11236351 & G & $A$ & 0.407 \\
\hline 11 & rs4100076 & C & $A$ & 0.1628 \\
\hline 11 & rs2712807 & G & $A$ & 0.3023 \\
\hline 11 & rs2851069 & C & $\mathrm{T}$ & 0.1744 \\
\hline 11 & rs2712810 & $T$ & $A$ & 0.3023 \\
\hline 11 & rs2712819 & G & $A$ & 0.3023 \\
\hline 11 & rs2712820 & $\mathrm{T}$ & C & 0.3023 \\
\hline 11 & rs 11236359 & A & G & 0.4767 \\
\hline 11 & rs1109407 & A & G & 0.1279 \\
\hline 11 & rs1789694 & $\mathrm{T}$ & C & 0.3256 \\
\hline 11 & rs 12422149 & A & G & 0.0697 \\
\hline 11 & rs61741839 & $T$ & C & 0.0465 \\
\hline 11 & rs1612859 & C & $\mathrm{T}$ & 0.3837 \\
\hline 11 & rs115881705 & C & $\mathrm{T}$ & 0.0814 \\
\hline 11 & rs142877598 & A & G & 0.1047 \\
\hline 11 & rs3824903 & C & A & 0.4535 \\
\hline 11 & rs114169536 & $\mathrm{T}$ & A & 0.1977 \\
\hline 11 & rs116456559 & A & G & 0.1047 \\
\hline 11 & rs112455521 & A & G & 0.0697 \\
\hline 11 & rs 139480360 & A & G & 0.0581 \\
\hline 11 & rs145026521 & C & $\mathrm{T}$ & 0.1047 \\
\hline 11 & rs149636191 & C & G & 0.1395 \\
\hline 11 & rs2306168 & C & $\mathrm{T}$ & 0.5 \\
\hline 11 & rs139408570 & G & $\mathrm{T}$ & 0.1395 \\
\hline 11 & rs200583779 & G & GT & 0.1395 \\
\hline 11 & rs190362624 & A & G & 0.1395 \\
\hline 11 & rs116211275 & G & A & 0.1395 \\
\hline 11 & rs114730634 & $\mathrm{T}$ & C & 0.1395 \\
\hline 11 & rs57141326 & A & G & 0.0814 \\
\hline 11 & rs3781727 & C & $\mathrm{T}$ & 0.1744 \\
\hline 11 & rs41298117 & G & C & 0.2442 \\
\hline 11 & rs1801906 & C & $\mathrm{T}$ & 0.4419 \\
\hline 11 & rs41298121 & C & $\mathrm{T}$ & 0.4419 \\
\hline 11 & rs17133818 & T & C & 0.1279 \\
\hline 11 & rs7951787 & G & A & 0.4302 \\
\hline 11 & rs143590827 & C & CA & 0.1395 \\
\hline 11 & rs74328774 & $A$ & G & 0.1395 \\
\hline 11 & rs57279023 & G & A & 0.4419 \\
\hline 11 & rs10793116 & G & A & 0.0581 \\
\hline 11 & rs115385770 & $\mathrm{T}$ & C & 0.1395 \\
\hline 11 & rs148725627 & $A$ & G & 0.1395 \\
\hline 11 & rs7924924 & C & $\mathrm{T}$ & 0.2558 \\
\hline 11 & rs137940642 & $A$ & G & 0.0581 \\
\hline 11 & rs77388683 & $\mathrm{T}$ & A & 0.1395 \\
\hline 11 & rs78028968 & C & $\mathrm{T}$ & 0.1395 \\
\hline 11 & rs10793117 & $\mathrm{T}$ & A & 0.0465 \\
\hline 11 & rs147451830 & G & A & 0.1395 \\
\hline
\end{tabular}

Table 1-A1 continues in the next column $\rightarrow$
Table 1-A1 (Continues...): Minor allele frequencies for 1846 polymorphisms in 43 black South Africans.

\begin{tabular}{|c|c|c|c|c|}
\hline Chromosome & Polymorphism & Minor allele & Major allele & $\begin{array}{l}\text { Minor allele } \\
\text { frequency }\end{array}$ \\
\hline 11 & rs1676889 & $\mathrm{T}$ & C & 0.0465 \\
\hline 11 & rs138139803 & $\mathrm{T}$ & C & 0.1395 \\
\hline 11 & rs138268018 & A & C & 0.1395 \\
\hline 11 & rs139792944 & G & $\mathrm{T}$ & 0.1395 \\
\hline 11 & rs1789679 & G & A & 0.0465 \\
\hline 11 & rs76510484 & G & C & 0.1395 \\
\hline 11 & rs11825144 & G & A & 0.0814 \\
\hline 11 & rs149949680 & C & $\mathrm{T}$ & 0.1395 \\
\hline 11 & rs145092162 & A & G & 0.0581 \\
\hline 11 & rs10899080 & A & G & 0.0465 \\
\hline 11 & rs115237508 & G & A & 0.1395 \\
\hline 11 & rs115316534 & $\mathrm{C}$ & $\mathrm{T}$ & 0.1395 \\
\hline 11 & rs144143765 & $\mathrm{T}$ & G & 0.1395 \\
\hline 11 & rs148442723 & $\mathrm{C}$ & $\mathrm{T}$ & 0.1395 \\
\hline 11 & rs142620661 & A & C & 0.1395 \\
\hline 11 & rs116234547 & $C$ & G & 0.1395 \\
\hline 11 & rs1676891 & C & A & 0.4767 \\
\hline 11 & rs116753650 & A & G & 0.1395 \\
\hline 11 & rs141666090 & $\mathrm{T}$ & G & 0.1395 \\
\hline 11 & rs114506297 & $\mathrm{T}$ & C & 0.1395 \\
\hline 11 & rs76519350 & A & G & 0.1395 \\
\hline 11 & rs150487846 & A & C & 0.1395 \\
\hline 11 & rs200183315 & G & $\mathrm{GC}$ & 0.1395 \\
\hline 11 & rs151119066 & $\mathrm{T}$ & $\mathrm{C}$ & 0.0465 \\
\hline 11 & rs145363174 & C & G & 0.1395 \\
\hline 11 & rs147485017 & G & A & 0.1395 \\
\hline 11 & rs78843443 & A & $\mathrm{T}$ & 0.1395 \\
\hline 11 & rs148791815 & A & $\mathrm{T}$ & 0.1395 \\
\hline 12 & rs4149032 & C & $\mathrm{T}$ & 0.1809 \\
\hline 12 & rs4149056 & - & $\mathrm{T}$ & 0.0 \\
\hline 12 & rs143218796 & A & G & 0.0697 \\
\hline 12 & rs4149069 & C & G & 0.4884 \\
\hline 12 & rs76715736 & A & G & 0.1047 \\
\hline 12 & rs11045870 & A & G & 0.0465 \\
\hline 12 & rs79494188 & $\mathrm{T}$ & A & 0.1047 \\
\hline 12 & rs11045871 & G & A & 0.0465 \\
\hline 12 & rs11045872 & G & A & 0.0465 \\
\hline 12 & rs75702089 & C & $\mathrm{T}$ & 0.0465 \\
\hline 12 & rs10841761 & C & G & 0.0465 \\
\hline 12 & rs76373857 & C & $\mathrm{T}$ & 0.1047 \\
\hline 12 & rs74690297 & G & C & 0.0465 \\
\hline 12 & rs11045873 & A & $\mathrm{T}$ & 0.0465 \\
\hline 12 & rs11045874 & $\mathrm{C}$ & G & 0.0930 \\
\hline 12 & rs4149079 & C & $\mathrm{T}$ & 0.1512 \\
\hline 12 & rs12814646 & A & C & 0.0465 \\
\hline 12 & rs72655362 & A & G & 0.1047 \\
\hline 12 & rs6487214 & A & C & 0.1512 \\
\hline 12 & rs7978219 & $\mathrm{C}$ & $\mathrm{T}$ & 0.1512 \\
\hline 12 & rs7965941 & G & A & 0.1512 \\
\hline 12 & rs58103058 & $\mathrm{T}$ & C & 0.0465 \\
\hline 12 & rs7966037 & G & A & 0.1512 \\
\hline 12 & rs7966129 & $\mathrm{T}$ & C & 0.1512 \\
\hline 12 & rs7966448 & A & G & 0.1512 \\
\hline 12 & rs7966269 & $\mathrm{T}$ & C & 0.1512 \\
\hline 12 & rs7966270 & $C$ & A & 0.0581 \\
\hline 12 & rs7966581 & A & G & 0.1512 \\
\hline 12 & rs7966613 & G & A & 0.1512 \\
\hline 12 & rs34882548 & $A C$ & A & 0.1512 \\
\hline 12 & rs76928130 & $T$ & C & 0.0814 \\
\hline 12 & rs61447271 & C & $\mathrm{T}$ & 0.0930 \\
\hline
\end{tabular}

Table 1-A1 continues on the next page $\rightarrow$ 
Table 1-A1 (Continues...): Minor allele frequencies for 1846 polymorphisms in 43 black South Africans.

\begin{tabular}{|c|c|c|c|c|}
\hline Chromosome & Polymorphism & Minor allele & Major allele & $\begin{array}{l}\text { Minor allele } \\
\text { frequency }\end{array}$ \\
\hline 12 & rs7953829 & G & $\mathrm{T}$ & 0.0581 \\
\hline 12 & rs183892220 & $\mathrm{T}$ & G & 0.0581 \\
\hline 12 & rs4149082 & G & C & 0.0930 \\
\hline 12 & rs58964144 & G & $\mathrm{T}$ & 0.0581 \\
\hline 12 & rs115108625 & $A$ & $\mathrm{~T}$ & 0.0581 \\
\hline 12 & rs11045877 & G & $\mathrm{T}$ & 0.0930 \\
\hline 12 & rs 12369359 & G & $T$ & 0.0930 \\
\hline 12 & rs71444108 & GAGT & CCACG & 0.2 \\
\hline 12 & rs78695636 & A & C & 0.1047 \\
\hline 12 & rs200875545 & C & CAT & 0.0814 \\
\hline 12 & rs10841763 & C & $\mathrm{T}$ & 0.0930 \\
\hline 12 & rs717959 & C & $\mathrm{T}$ & 0.0930 \\
\hline 12 & rs115913130 & C & $\mathrm{T}$ & 0.0697 \\
\hline 12 & rs116180575 & $A$ & G & 0.0697 \\
\hline 12 & rs115108635 & $T$ & C & 0.0697 \\
\hline 12 & rs3962562 & $\mathrm{T}$ & G & 0.1628 \\
\hline 12 & rs 11045881 & C & $\mathrm{T}$ & 0.1628 \\
\hline 12 & rs138156422 & C & G & 0.0814 \\
\hline 12 & rs11045882 & $T$ & C & 0.2674 \\
\hline 12 & rs11045883 & A & G & 0.0930 \\
\hline 12 & rs 10841764 & G & C & 0.1628 \\
\hline 12 & rs10841765 & $\mathrm{T}$ & C & 0.0930 \\
\hline 12 & rs111359254 & C & $\mathrm{T}$ & 0.0697 \\
\hline 12 & rs77857357 & A & G & 0.0697 \\
\hline 12 & rs78545516 & $T$ & C & 0.1047 \\
\hline 12 & rs10841767 & G & A & 0.0465 \\
\hline 12 & rs10841768 & C & A & 0.0465 \\
\hline 12 & rs11513225 & C & $\mathrm{T}$ & 0.0697 \\
\hline 12 & rs116472641 & G & C & 0.1047 \\
\hline 12 & rs146059821 & $\mathrm{T}$ & A & 0.0697 \\
\hline 12 & rs201304263 & AG & A & 0.0465 \\
\hline 12 & rs12829704 & A & G & 0.0465 \\
\hline 12 & rs143654242 & G & $\mathrm{T}$ & 0.0465 \\
\hline 12 & rs116166170 & G & A & 0.1047 \\
\hline 12 & rs11045890 & T & C & 0.0697 \\
\hline 12 & rs56164184 & C & $\mathrm{T}$ & 0.0465 \\
\hline 12 & rs200739289 & CT & C & 0.1395 \\
\hline 12 & rs56370646 & TA & $\mathrm{T}$ & 0.1395 \\
\hline 12 & rs 12578392 & C & $\mathrm{T}$ & 0.1395 \\
\hline 12 & rs57130116 & A & G & 0.1395 \\
\hline 12 & rs34111581 & C & $\mathrm{T}$ & 0.0465 \\
\hline 12 & rs112848945 & $\mathrm{T}$ & A & 0.0465 \\
\hline 12 & rs 11615107 & $\mathrm{~T}$ & C & 0.1047 \\
\hline 12 & rs12371604 & C & $\mathrm{T}$ & 0.0930 \\
\hline 12 & rs12815795 & C & $\mathrm{T}$ & 0.0465 \\
\hline 12 & rs34671512 & C & A & 0.1047 \\
\hline 12 & rs72655363 & T & C & 0.1047 \\
\hline 12 & rs4149087 & G & $\mathrm{T}$ & 0.1395 \\
\hline 12 & rs4149088 & G & A & 0.1395 \\
\hline 12 & rs11045892 & G & A & 0.0465 \\
\hline 12 & rs 11045893 & C & $\mathrm{T}$ & 0.0465 \\
\hline 12 & rs 12372157 & G & $\mathrm{T}$ & 0.1395 \\
\hline 12 & rs11045895 & C & $\mathrm{T}$ & 0.0814 \\
\hline 12 & rs77757956 & $\mathrm{T}$ & C & 0.0697 \\
\hline 12 & rs 12370842 & A & G & 0.0465 \\
\hline 12 & rs11045896 & C & A & 0.0930 \\
\hline 12 & rs111273303 & G & A & 0.1047 \\
\hline 12 & rs115431317 & C & A & 0.0697 \\
\hline 12 & rs111436442 & G & A & 0.1047 \\
\hline 12 & rs200689244 & G & GT & 0.0465 \\
\hline
\end{tabular}

Table 1-A1 continues in the next column $\rightarrow$
Table 1-A1 (Continues...): Minor allele frequencies for 1846 polymorphisms in 43 black South Africans.

\begin{tabular}{|c|c|c|c|c|}
\hline Chromosome & Polymorphism & Minor allele & Major allele & $\begin{array}{l}\text { Minor allele } \\
\text { frequency }\end{array}$ \\
\hline 12 & rs12372593 & A & $\mathrm{T}$ & 0.0465 \\
\hline 12 & rs74064264 & A & C & 0.0465 \\
\hline 12 & rs10841769 & A & G & 0.3256 \\
\hline 12 & rs77088330 & C & $\mathrm{T}$ & 0.0697 \\
\hline 12 & rs116428713 & C & A & 0.1047 \\
\hline 12 & rs56397921 & G & C & 0.0465 \\
\hline 12 & rs80168561 & C & A & 0.0697 \\
\hline 12 & rs7960688 & C & G & 0.0581 \\
\hline 12 & rs7960384 & G & A & 0.0581 \\
\hline 12 & rs79458194 & A & C & 0.1047 \\
\hline 12 & rs78178574 & $\mathrm{T}$ & G & 0.1047 \\
\hline 12 & rs78083330 & C & $\mathrm{T}$ & 0.1047 \\
\hline 12 & rs58173663 & G & C & 0.0465 \\
\hline 12 & rs56290140 & TTAG & $\mathrm{T}$ & 0.4884 \\
\hline 12 & rs73248969 & $\mathrm{T}$ & C & 0.0581 \\
\hline 12 & rs55773124 & $C$ & A & 0.0581 \\
\hline 12 & rs11045900 & G & A & 0.1395 \\
\hline 12 & rs11045901 & C & $\mathrm{T}$ & 0.1395 \\
\hline 12 & rs55941487 & $\mathrm{T}$ & C & 0.0465 \\
\hline 12 & rs114917941 & C & A & 0.1047 \\
\hline 12 & rs77256705 & G & C & 0.0465 \\
\hline 12 & rs59938822 & G & A & 0.1047 \\
\hline 12 & rs201721678 & A & ATAACT & 0.104 \\
\hline 12 & rs79036466 & G & $\mathrm{T}$ & 0.1047 \\
\hline 12 & rs74064273 & C & G & 0.0465 \\
\hline 12 & rs73069028 & A & G & 0.0697 \\
\hline 12 & rs35325400 & C & $\mathrm{T}$ & 0.1977 \\
\hline 12 & rs7975166 & A & G & 0.1047 \\
\hline 12 & rs35995344 & C & $\mathrm{T}$ & 0.0697 \\
\hline 12 & rs144440970 & $\mathrm{T}$ & C & 0.1047 \\
\hline 12 & rs144019604 & G & $\mathrm{T}$ & 0.1047 \\
\hline 12 & rs77989963 & C & $\mathrm{T}$ & 0.0697 \\
\hline 12 & rs11045906 & G & A & 0.2558 \\
\hline 12 & rs12427132 & G & A & 0.0697 \\
\hline 12 & rs145947620 & $\mathrm{T}$ & G & 0.0697 \\
\hline 12 & rs148455728 & A & G & 0.0697 \\
\hline 12 & rs75967989 & G & A & 0.0581 \\
\hline 12 & rs10841778 & G & $\mathrm{T}$ & 0.0814 \\
\hline 12 & rs10841779 & C & $\mathrm{T}$ & 0.1512 \\
\hline 12 & rs10841780 & $\mathrm{T}$ & C & 0.1512 \\
\hline 12 & rs71446763 & A & G & 0.0581 \\
\hline 12 & rs73250843 & A & G & 0.1047 \\
\hline 12 & rs111512821 & C & $\mathrm{T}$ & 0.0697 \\
\hline 12 & rs10841781 & G & A & 0.2791 \\
\hline 12 & rs142851022 & G & A & 0.0697 \\
\hline 12 & rs4149009 & C & $\mathrm{T}$ & 0.3256 \\
\hline 12 & rs78801100 & $\mathrm{T}$ & C & 0.0697 \\
\hline 12 & rs147803163 & A & ATTTGCTTTC & 0.1512 \\
\hline 12 & rs4149008 & G & A & 0.3837 \\
\hline 12 & rs4149007 & $\mathrm{T}$ & C & 0.1512 \\
\hline 12 & rs12317843 & C & $\mathrm{T}$ & 0.4767 \\
\hline 12 & rs73250847 & G & A & 0.1395 \\
\hline 12 & rs4149006 & $\mathrm{T}$ & G & 0.186 \\
\hline 12 & rs112403792 & $\mathrm{T}$ & C & 0.1744 \\
\hline 12 & rs11045919 & G & $\mathrm{T}$ & 0.4767 \\
\hline 12 & rs4140389 & G & $\mathrm{T}$ & 0.4186 \\
\hline 12 & rs11045920 & A & C & 0.0581 \\
\hline 12 & rs11568557 & $\mathrm{C}$ & G & 0.0581 \\
\hline 12 & rs34636443 & ATAAC & A & 0.4186 \\
\hline 12 & rs202105274 & ATAAG & $\mathrm{T}$ & 0.0581 \\
\hline
\end{tabular}

Table 1-A1 continues on the next page $\rightarrow$ 
Table 1-A1 (Continues...): Minor allele frequencies for 1846 polymorphisms in 43 black South Africans.

\begin{tabular}{|c|c|c|c|c|}
\hline Chromosome & Polymorphism & Minor allele & Major allele & $\begin{array}{l}\text { Minor allele } \\
\text { frequency }\end{array}$ \\
\hline 12 & rs 12230401 & C & G & 0.1512 \\
\hline 12 & rs57899519 & $A$ & $\mathrm{~T}$ & 0.4186 \\
\hline 12 & rs16923597 & A & G & 0.4186 \\
\hline 12 & rs7980842 & $\mathrm{T}$ & G & 0.2093 \\
\hline 12 & rs7967354 & $T$ & C & 0.3837 \\
\hline 12 & rs10841782 & $\mathrm{T}$ & C & 0.2093 \\
\hline 12 & rs190500283 & A & G & 0.0697 \\
\hline 12 & rs7955581 & C & G & 0.3721 \\
\hline 12 & rs150018371 & GAGAT & G & 0.4186 \\
\hline 12 & rs2417971 & G & A & 0.3256 \\
\hline 12 & rs11045922 & C & G & 0.3837 \\
\hline 12 & rs11045923 & C & G & 0.3605 \\
\hline 12 & rs16923608 & G & $\mathrm{T}$ & 0.3837 \\
\hline 12 & rs183479283 & $A$ & G & 0.0697 \\
\hline 12 & rs12298817 & C & $\mathrm{T}$ & 0.4186 \\
\hline 12 & rs143783884 & C & $A$ & 0.0697 \\
\hline 12 & rs58923303 & $\mathrm{T}$ & TGA & 0.1395 \\
\hline 12 & rs12300594 & C & $\mathrm{T}$ & 0.4186 \\
\hline 12 & rs57503786 & $T$ & G & 0.2093 \\
\hline 12 & rs201191507 & AAGT & G & 0.0581 \\
\hline 12 & rs4149005 & G & $\mathrm{T}$ & 0.3721 \\
\hline 12 & rs4149004 & C & $\mathrm{T}$ & 0.0581 \\
\hline 12 & rs3736081 & C & $\mathrm{T}$ & 0.0581 \\
\hline 12 & rs12303996 & C & $\mathrm{T}$ & 0.1977 \\
\hline 12 & rs 12297072 & A & G & 0.0930 \\
\hline 12 & rs2199688 & G & $\mathrm{T}$ & 0.3837 \\
\hline 12 & rs11568565 & A & G & 0.0581 \\
\hline 12 & rs3764044 & C & $\mathrm{T}$ & 0.1395 \\
\hline 12 & rs11829484 & $\mathrm{T}$ & C & 0.2093 \\
\hline 12 & rs112034436 & A & G & 0.0581 \\
\hline 12 & rs 10431251 & $\mathrm{~T}$ & C & 0.4186 \\
\hline 12 & rs11045926 & C & $\mathrm{T}$ & 0.4186 \\
\hline 12 & rs11045927 & G & $\mathrm{T}$ & 0.4186 \\
\hline 12 & rs184309917 & C & $\mathrm{T}$ & 0.0697 \\
\hline 12 & rs112346601 & C & G & 0.0581 \\
\hline 12 & rs113601942 & $\mathrm{T}$ & C & 0.0581 \\
\hline 12 & rs116763958 & C & $\mathrm{T}$ & 0.0697 \\
\hline 12 & rs4453284 & C & G & 0.0581 \\
\hline 12 & rs61926248 & G & C & 0.2442 \\
\hline 12 & rs143498446 & A & G & 0.0697 \\
\hline 12 & rs112699138 & A & C & 0.0581 \\
\hline 12 & rs7316412 & G & C & 0.2209 \\
\hline 12 & rs73250866 & C & G & 0.1628 \\
\hline 12 & rs2199687 & A & $\mathrm{T}$ & 0.3837 \\
\hline 12 & rs112225684 & A & G & 0.2209 \\
\hline 12 & rs11045930 & $\mathrm{T}$ & C & 0.407 \\
\hline 12 & rs77209586 & C & $\mathrm{T}$ & 0.0697 \\
\hline 12 & rs60594228 & C & $\mathrm{T}$ & 0.1395 \\
\hline 12 & rs11535999 & A & C & 0.3256 \\
\hline 12 & rs116452856 & C & $\mathrm{T}$ & 0.1744 \\
\hline 12 & rs2417972 & C & $\mathrm{T}$ & 0.407 \\
\hline 12 & rs 113602450 & C & $\mathrm{T}$ & 0.0581 \\
\hline 12 & rs11833627 & C & $\mathrm{T}$ & 0.314 \\
\hline 12 & rs112261153 & G & $\mathrm{T}$ & 0.0581 \\
\hline 12 & rs11533477 & $A$ & G & 0.0581 \\
\hline 12 & rs60098288 & C & G & 0.0814 \\
\hline 12 & rs184036934 & $\mathrm{T}$ & C & 0.0581 \\
\hline 12 & rs61552073 & A & G & 0.1395 \\
\hline 12 & rs11045941 & G & A & 0.1279 \\
\hline 12 & rs79349376 & $\mathrm{T}$ & A & 0.0581 \\
\hline
\end{tabular}

Table 1-A1 continues in the next column $\rightarrow$
Table 1-A1 (Continues...): Minor allele frequencies for 1846 polymorphisms in 43 black South Africans.

\begin{tabular}{|c|c|c|c|c|}
\hline Chromosome & Polymorphism & Minor allele & Major allele & $\begin{array}{l}\text { Minor allele } \\
\text { frequency }\end{array}$ \\
\hline 12 & rs66777941 & $\mathrm{C}$ & $\mathrm{T}$ & 0.3372 \\
\hline 12 & rs199940208 & CGT & C & 0.1744 \\
\hline 12 & rs10841786 & A & $\mathrm{T}$ & 0.3256 \\
\hline 12 & rs10841787 & C & A & 0.3256 \\
\hline 12 & rs74064507 & $\mathrm{T}$ & A & 0.0581 \\
\hline 12 & rs7305484 & A & G & 0.1744 \\
\hline 12 & rs4149003 & C & $\mathrm{T}$ & 0.2558 \\
\hline 12 & rs74064510 & $\mathrm{T}$ & G & 0.0697 \\
\hline 12 & rs16923623 & G & A & 0.0697 \\
\hline 12 & rs6487215 & A & G & 0.3372 \\
\hline 12 & rs7977483 & G & A & 0.1395 \\
\hline 12 & rs116040707 & G & A & 0.1163 \\
\hline 12 & rs79445965 & A & G & 0.0581 \\
\hline 12 & rs7978322 & $\mathrm{T}$ & G & 0.3372 \\
\hline 12 & rs78735020 & A & G & 0.2558 \\
\hline 12 & rs112828357 & $\mathrm{T}$ & C & 0.1744 \\
\hline 12 & rs10770795 & A & G & 0.3837 \\
\hline 12 & rs139780426 & A & AAAAT & 0.3488 \\
\hline 12 & rs2900480 & G & C & 0.3488 \\
\hline 12 & rs11568574 & C & $\mathrm{T}$ & 0.0581 \\
\hline 12 & rs11568573 & G & A & 0.1744 \\
\hline 12 & rs1000626 & G & A & 0.0930 \\
\hline 12 & rs59896213 & C & $\mathrm{CTT}$ & 0.186 \\
\hline 12 & rs144566343 & C & $\mathrm{G}$ & 0.0697 \\
\hline 12 & rs4522218 & G & $\mathrm{T}$ & 0.0697 \\
\hline 12 & rs4148996 & G & C & 0.2093 \\
\hline 12 & rs2417973 & $\mathrm{T}$ & G & 0.2093 \\
\hline 12 & rs7952736 & A & G & 0.1744 \\
\hline 12 & rs145614255 & A & AACAATTCT & 0.2093 \\
\hline 12 & rs74064512 & $\mathrm{T}$ & C & 0.0581 \\
\hline 12 & rs139863986 & CA & C & 0.2093 \\
\hline 12 & rs11045948 & A & G & 0.0581 \\
\hline 12 & rs73235208 & $\mathrm{T}$ & G & 0.2093 \\
\hline 12 & rs199525542 & GTA & G & 0.2093 \\
\hline 12 & rs199877480 & C & ССТСТ & 0.0697 \\
\hline 12 & rs143814959 & ATG & A & 0.407 \\
\hline 12 & rs148416055 & A & ATATG & 0.1395 \\
\hline 12 & rs113781670 & G & A & 0.1744 \\
\hline 12 & rs11568550 & A & G & 0.0581 \\
\hline 12 & rs4148995 & $C$ & $\mathrm{~T}$ & 0.0581 \\
\hline 12 & rs58957989 & GT & G & 0.1977 \\
\hline 12 & rs11832851 & A & G & 0.1977 \\
\hline 12 & rs11045955 & $\mathrm{T}$ & A & 0.2093 \\
\hline 12 & rs111334847 & $\mathrm{T}$ & C & 0.1395 \\
\hline 12 & rs142350951 & $\mathrm{T}$ & C & 0.0697 \\
\hline 12 & rs11045956 & G & A & 0.1744 \\
\hline 12 & rs74064514 & C & A & 0.1279 \\
\hline 12 & rs74064516 & G & A & 0.1279 \\
\hline 12 & rs114925264 & G & A & 0.0581 \\
\hline 12 & rs11045958 & G & A & 0.186 \\
\hline 12 & rs11045959 & $\mathrm{T}$ & C & 0.1395 \\
\hline 12 & rs77676075 & A & G & 0.1744 \\
\hline 12 & rs113810619 & A & G & 0.1744 \\
\hline 12 & rs112633391 & C & A & 0.1744 \\
\hline 12 & rs11838023 & C & $\mathrm{T}$ & 0.2674 \\
\hline 12 & rs3838822 & $\mathrm{C}$ & CTG & 0.1163 \\
\hline 12 & rs57472326 & A & G & 0.3256 \\
\hline 12 & rs7305911 & $\mathrm{T}$ & C & 0.3256 \\
\hline 12 & rs151134208 & C & $\mathrm{T}$ & 0.0697 \\
\hline 12 & rs200037623 & $\mathrm{T}$ & TTAA & 0.0581 \\
\hline
\end{tabular}

Table 1-A1 continues on the next page $\rightarrow$ 
Table 1-A1 (Continues...): Minor allele frequencies for 1846 polymorphisms in 43 black South Africans.

\begin{tabular}{|c|c|c|c|c|}
\hline Chromosome & Polymorphism & Minor allele & Major allele & $\begin{array}{l}\text { Minor allele } \\
\text { frequency }\end{array}$ \\
\hline 12 & rs148368950 & $T$ & TAATA & 0.0581 \\
\hline 12 & rs80225829 & C & $\mathrm{T}$ & 0.0697 \\
\hline 12 & rs111254386 & G & $A$ & 0.1744 \\
\hline 12 & rs11568567 & $A$ & $\mathrm{~T}$ & 0.0697 \\
\hline 12 & rs4148994 & $T$ & C & 0.0581 \\
\hline 12 & rs 11045960 & $A$ & G & 0.1744 \\
\hline 12 & rs4148993 & G & A & 0.3256 \\
\hline 12 & rs11836396 & C & $\mathrm{T}$ & 0.2674 \\
\hline 12 & rs113449898 & C & $\mathrm{T}$ & 0.1744 \\
\hline 12 & rs34211424 & G & A & 0.1744 \\
\hline 12 & rs 112130054 & G & C & 0.1744 \\
\hline 12 & rs148678312 & A & AAC & 0.1744 \\
\hline 12 & rs12314183 & $A$ & G & 0.2791 \\
\hline 12 & rs2417974 & C & $\mathrm{T}$ & 0.0581 \\
\hline 12 & rs2127117 & $T$ & C & 0.1163 \\
\hline 12 & rs2169883 & G & $A$ & 0.1163 \\
\hline 12 & rs7304940 & C & $\mathrm{T}$ & 0.1744 \\
\hline 12 & rs112297403 & A & G & 0.1977 \\
\hline 12 & rs199881908 & A & AGAG & 0.0581 \\
\hline 12 & rs142521520 & CA & $\mathrm{C}$ & 0.1163 \\
\hline 12 & rs142749463 & $T$ & C & 0.0697 \\
\hline 12 & rs12231484 & A & C & 0.0581 \\
\hline 12 & rs2306226 & C & $\mathrm{T}$ & 0.0581 \\
\hline 12 & rs10841789 & C & $A$ & 0.1047 \\
\hline 12 & rs58587133 & C & $\mathrm{T}$ & 0.0814 \\
\hline 12 & rs61927778 & $\mathrm{T}$ & C & 0.0697 \\
\hline 12 & rs7137014 & C & $\mathrm{T}$ & 0.1744 \\
\hline 12 & rs61537911 & C & $\mathrm{T}$ & 0.1512 \\
\hline 12 & rs77335871 & C & $\mathrm{T}$ & 0.0581 \\
\hline 12 & rs7974575 & A & G & 0.1047 \\
\hline 12 & rs12228765 & C & $\mathrm{T}$ & 0.0581 \\
\hline 12 & rs 12227319 & C & G & 0.2907 \\
\hline 12 & rs34249976 & C & $\mathrm{T}$ & 0.1744 \\
\hline 12 & rs112214775 & A & C & 0.1977 \\
\hline 12 & rs59940564 & A & $\mathrm{T}$ & 0.1512 \\
\hline 12 & rs199577219 & GA & G & 0.1628 \\
\hline 12 & rs73237221 & $T$ & $A$ & 0.0814 \\
\hline 12 & rs11045966 & $\mathrm{T}$ & C & 0.1163 \\
\hline 12 & rs58406283 & A & G & 0.0930 \\
\hline 12 & rs2219793 & G & $\mathrm{T}$ & 0.2907 \\
\hline 12 & rs 11568570 & A & $\mathrm{T}$ & 0.0581 \\
\hline 12 & rs2219792 & C & G & 0.0697 \\
\hline 12 & rs 10505872 & A & G & 0.0930 \\
\hline 12 & rs76669231 & A & G & 0.0581 \\
\hline 12 & rs11045969 & A & C & 0.0581 \\
\hline 12 & rs11833771 & G & A & 0.1512 \\
\hline 12 & rs11045971 & C & G & 0.0581 \\
\hline 12 & rs 10082739 & $\mathrm{~T}$ & C & 0.0465 \\
\hline 12 & rs12298237 & T & C & 0.1977 \\
\hline 12 & rs7312628 & $\mathrm{T}$ & C & 0.1512 \\
\hline 12 & rs61927780 & A & G & 0.0930 \\
\hline 12 & rs61927781 & C & $\mathrm{T}$ & 0.0697 \\
\hline 12 & rs139621920 & TA & $\mathrm{T}$ & 0.0697 \\
\hline 12 & rs11830993 & $\mathrm{T}$ & C & 0.1395 \\
\hline 12 & rs 4078 & G & A & 0.0930 \\
\hline 12 & rs77207093 & $\mathrm{T}$ & A & 0.0930 \\
\hline 12 & rs113307426 & G & A & 0.0581 \\
\hline 12 & rs61927782 & A & G & 0.0930 \\
\hline 12 & rs11836945 & C & A & 0.1512 \\
\hline 12 & rs61927783 & T & C & 0.0930 \\
\hline
\end{tabular}

Table 1-A1 continues in the next column $\rightarrow$
Table 1-A1 (Continues...): Minor allele frequencies for 1846 polymorphisms in 43 black South Africans.

\begin{tabular}{|c|c|c|c|c|}
\hline Chromosome & Polymorphism & Minor allele & Major allele & $\begin{array}{l}\text { Minor allele } \\
\text { frequency }\end{array}$ \\
\hline 12 & rs34696667 & A & AT & 0.1512 \\
\hline 12 & rs11832394 & $\mathrm{T}$ & C & 0.1512 \\
\hline 12 & rs73237235 & G & A & 0.1512 \\
\hline 12 & rs114032998 & $A$ & G & 0.0697 \\
\hline 12 & rs2199683 & A & C & 0.1512 \\
\hline 12 & rs113467449 & $\mathrm{T}$ & C & 0.1512 \\
\hline 12 & rs143459704 & C & CCCCA & 0.1512 \\
\hline 12 & rs11830939 & $\mathrm{T}$ & G & 0.1512 \\
\hline 12 & rs4148987 & A & G & 0.4884 \\
\hline 12 & rs151247881 & AAAAT & A & 0.1512 \\
\hline 12 & rs115692334 & $\mathrm{T}$ & A & 0.0581 \\
\hline 12 & rs61927786 & $\mathrm{C}$ & $\mathrm{T}$ & 0.0930 \\
\hline 12 & rs11832186 & G & C & 0.1512 \\
\hline 12 & rs61927787 & $\mathrm{T}$ & A & 0.0930 \\
\hline 12 & rs61927788 & $\mathrm{T}$ & C & 0.0930 \\
\hline 12 & rs73237241 & C & A & 0.1512 \\
\hline 12 & rs61927789 & G & A & 0.0930 \\
\hline 12 & rs112737048 & G & C & 0.0581 \\
\hline 12 & rs143086486 & G & $\mathrm{T}$ & 0.0581 \\
\hline 12 & rs112749642 & $\mathrm{T}$ & C & 0.0581 \\
\hline 12 & rs113158516 & $\mathrm{T}$ & G & 0.0581 \\
\hline 12 & rs7968842 & G & A & 0.3372 \\
\hline 12 & rs4148986 & G & A & 0.3372 \\
\hline 12 & rs4148985 & C & $\mathrm{T}$ & 0.3372 \\
\hline 12 & rs4148984 & A & G & 0.3372 \\
\hline 12 & rs4148983 & G & A & 0.3372 \\
\hline 12 & rs4148982 & $\mathrm{T}$ & C & 0.3372 \\
\hline 12 & rs4148981 & C & $\mathrm{T}$ & 0.3372 \\
\hline 12 & rs3834939 & C & $\mathrm{CT}$ & 0.3372 \\
\hline 12 & rs141493226 & TATGTAC & $\mathrm{T}$ & 0.1512 \\
\hline 12 & rs7980167 & T & A & 0.0697 \\
\hline 12 & rs4148978 & C & $\mathrm{T}$ & 0.3372 \\
\hline 12 & rs4148977 & C & $\mathrm{T}$ & 0.3372 \\
\hline 12 & rs7980490 & $\mathrm{T}$ & C & 0.0930 \\
\hline 12 & rs3764042 & G & A & 0.3372 \\
\hline 12 & rs12230953 & C & $\mathrm{T}$ & 0.0814 \\
\hline 12 & rs 2857468 & A & $\mathrm{T}$ & 0.3837 \\
\hline 12 & rs4762818 & A & G & 0.2674 \\
\hline 12 & rs11830638 & G & A & 0.2674 \\
\hline 12 & rs11838314 & C & $\mathrm{T}$ & 0.2674 \\
\hline 12 & rs7954757 & G & A & 0.2674 \\
\hline 12 & rs1871288 & A & $\mathrm{T}$ & 0.0581 \\
\hline 12 & rs56348138 & $\mathrm{T}$ & C & 0.2791 \\
\hline 12 & rs11836617 & A & G & 0.2674 \\
\hline 12 & rs7138451 & G & A & 0.0697 \\
\hline 12 & rs73069061 & A & G & 0.2791 \\
\hline 12 & rs1979405 & C & $\mathrm{T}$ & 0.0581 \\
\hline 12 & rs56246579 & G & A & 0.2791 \\
\hline 12 & rs56806648 & $\mathrm{T}$ & C & 0.2791 \\
\hline 12 & rs115309986 & C & $\mathrm{T}$ & 0.0814 \\
\hline 12 & rs75208026 & C & $\mathrm{T}$ & 0.0930 \\
\hline 12 & rs2061825 & $\mathrm{T}$ & C & 0.0581 \\
\hline 12 & rs12809856 & $\mathrm{T}$ & C & 0.4767 \\
\hline 12 & rs12296154 & C & $\mathrm{T}$ & 0.3488 \\
\hline 12 & rs115685216 & G & A & 0.0814 \\
\hline 12 & rs10743413 & C & $\mathrm{T}$ & 0.3721 \\
\hline 12 & rs11045994 & C & $\mathrm{T}$ & 0.2791 \\
\hline 12 & rs11045995 & C & $\mathrm{T}$ & 0.0581 \\
\hline 12 & rs10770800 & G & A & 0.0465 \\
\hline 12 & rs73069071 & C & $\mathrm{T}$ & 0.3837 \\
\hline
\end{tabular}

Table 1-A1 continues on the next page $\rightarrow$ 
Table 1-A1 (Continues...): Minor allele frequencies for 1846 polymorphisms in 43 black South Africans.

\begin{tabular}{|c|c|c|c|c|}
\hline Chromosome & Polymorphism & Minor allele & Major allele & $\begin{array}{l}\text { Minor allele } \\
\text { frequency }\end{array}$ \\
\hline 12 & rs77397980 & $\mathrm{C}$ & $\mathrm{T}$ & 0.0581 \\
\hline 12 & rs114958964 & C & $\mathrm{T}$ & 0.1512 \\
\hline 12 & rs114486873 & G & A & 0.1512 \\
\hline 12 & rs63378661 & G & A & 0.1512 \\
\hline 12 & rs140377659 & C & CAATGAGAAAAA & 0.1744 \\
\hline 12 & rs78331403 & A & G & 0.1163 \\
\hline 12 & rs5484 & $T$ & C & 0.1744 \\
\hline 12 & rs5486 & G & A & 0.0581 \\
\hline 12 & rs1056007 & $\mathrm{T}$ & G & 0.1744 \\
\hline 12 & rs5488 & $A$ & $\mathrm{~T}$ & 0.1744 \\
\hline 12 & rs3213208 & G & $\mathrm{T}$ & 0.1163 \\
\hline 12 & rs 12826421 & C & G & 0.1744 \\
\hline 12 & rs 12833409 & $T$ & C & 0.1163 \\
\hline 12 & rs4762700 & C & $\mathrm{T}$ & 0.4767 \\
\hline 12 & rs55785938 & A & G & 0.0581 \\
\hline 12 & rs 12317073 & A & G & 0.4767 \\
\hline 13 & rs73551639 & A & $\mathrm{T}$ & 0.2442 \\
\hline 13 & rs73551640 & G & $\mathrm{T}$ & 0.2442 \\
\hline 13 & rs73551643 & A & G & 0.2442 \\
\hline 13 & rs73551645 & A & G & 0.2442 \\
\hline 13 & rs115744968 & G & C & 0.1628 \\
\hline 13 & rs9516500 & A & G & 0.1279 \\
\hline 13 & rs73551647 & A & C & 0.2442 \\
\hline 13 & rs60329452 & $\mathrm{T}$ & C & 0.1628 \\
\hline 13 & rs9524719 & A & G & 0.1279 \\
\hline 13 & rs11841559 & G & C & 0.2442 \\
\hline 13 & rs11841597 & G & C & 0.2442 \\
\hline 13 & rs10508010 & C & A & 0.1279 \\
\hline 13 & rs113234367 & A & C & 0.1628 \\
\hline 13 & rs113546805 & $\mathrm{T}$ & C & 0.1628 \\
\hline 13 & rs 112160020 & A & $\mathrm{T}$ & 0.1628 \\
\hline 13 & rs7996263 & $\mathrm{T}$ & G & 0.2442 \\
\hline 13 & rs7998670 & G & A & 0.2442 \\
\hline 13 & rs113814775 & A & G & 0.407 \\
\hline 13 & rs73551665 & C & G & 0.2442 \\
\hline 13 & rs111480403 & A & G & 0.1628 \\
\hline 13 & rs 8002563 & A & G & 0.407 \\
\hline 13 & rs7981159 & A & G & 0.2442 \\
\hline 13 & rs9590149 & G & C & 0.4302 \\
\hline 13 & rs9590150 & $\mathrm{T}$ & A & 0.3372 \\
\hline 13 & rs6492760 & $\mathrm{T}$ & C & 0.2442 \\
\hline 13 & rs1925879 & C & G & 0.4302 \\
\hline 13 & rs1925878 & C & $\mathrm{T}$ & 0.4302 \\
\hline 13 & rs1925877 & A & G & 0.2674 \\
\hline 13 & rs200517290 & A & AT & 0.1628 \\
\hline 13 & rs9302029 & C & $\mathrm{T}$ & 0.4302 \\
\hline 13 & rs149953938 & A & G & 0.1628 \\
\hline 13 & rs9302030 & C & $\mathrm{T}$ & 0.4302 \\
\hline 13 & rs9302031 & C & $\mathrm{T}$ & 0.4302 \\
\hline 13 & rs116317258 & $\mathrm{T}$ & C & 0.1628 \\
\hline 13 & rs1925876 & C & $\mathrm{T}$ & 0.4302 \\
\hline 13 & rs1925875 & G & A & 0.2674 \\
\hline 13 & rs112661898 & $\mathrm{T}$ & A & 0.1628 \\
\hline 13 & rs16950404 & C & $\mathrm{T}$ & 0.1628 \\
\hline 13 & rs2148066 & G & A & 0.4302 \\
\hline 13 & rs2148065 & $\mathrm{T}$ & C & 0.4302 \\
\hline 13 & rs1952108 & G & A & 0.4302 \\
\hline 13 & rs2148064 & G & A & 0.4302 \\
\hline 13 & rs113589545 & $\mathrm{T}$ & C & 0.2442 \\
\hline 13 & rs141106024 & $\mathrm{T}$ & $\mathrm{C}$ & 0.1628 \\
\hline
\end{tabular}

Table 1-A1 continues in the next column $\rightarrow$
Table 1-A1 (Continues...): Minor allele frequencies for 1846 polymorphisms in 43 black South Africans.

\begin{tabular}{|c|c|c|c|c|}
\hline Chromosome & Polymorphism & Minor allele & Major allele & $\begin{array}{l}\text { Minor allele } \\
\text { frequency }\end{array}$ \\
\hline 13 & rs1925874 & A & G & 0.2674 \\
\hline 13 & rs41386448 & $\mathrm{T}$ & C & 0.1628 \\
\hline 13 & rs1925872 & C & A & 0.0697 \\
\hline 13 & rs5805894 & AT & A & 0.4302 \\
\hline 13 & rs1925870 & $\mathrm{T}$ & C & 0.4302 \\
\hline 13 & rs9524731 & $\mathrm{T}$ & $A$ & 0.4302 \\
\hline 13 & rs9524732 & $\mathrm{T}$ & C & 0.1628 \\
\hline 13 & rs9302032 & $A$ & $\mathrm{~T}$ & 0.186 \\
\hline 13 & rs72640796 & C & G & 0.1628 \\
\hline 13 & rs1925868 & A & G & 0.3372 \\
\hline 13 & rs2209537 & $A$ & G & 0.3372 \\
\hline 13 & rs34758312 & A & G & 0.0814 \\
\hline 13 & rs1925861 & A & G & 0.1628 \\
\hline 13 & rs1925860 & $\mathrm{T}$ & C & 0.1628 \\
\hline 13 & rs1925858 & $\mathrm{T}$ & C & 0.1628 \\
\hline 13 & rs61965532 & A & G & 0.1395 \\
\hline 13 & rs61965533 & C & $\mathrm{T}$ & 0.1395 \\
\hline 13 & rs9561747 & C & $\mathrm{T}$ & 0.1395 \\
\hline 13 & rs72642313 & $\mathrm{T}$ & C & 0.1628 \\
\hline 13 & rs9556446 & $C$ & G & 0.1395 \\
\hline 13 & rs9561749 & A & G & 0.1395 \\
\hline 13 & rs9556447 & A & G & 0.1395 \\
\hline 13 & rs9561750 & $\mathrm{T}$ & A & 0.1395 \\
\hline 13 & rs9561751 & G & $\mathrm{T}$ & 0.1395 \\
\hline 13 & rs9561752 & A & G & 0.1395 \\
\hline 13 & rs9561753 & G & C & 0.1395 \\
\hline 13 & rs34067449 & $\mathrm{T}$ & A & 0.0814 \\
\hline 13 & rs61965534 & G & A & 0.1395 \\
\hline 13 & rs59003216 & A & G & 0.1395 \\
\hline 13 & rs71854688 & $\mathrm{T}$ & TTAAGA & 0.139 \\
\hline 13 & rs9561758 & A & G & 0.1395 \\
\hline 13 & rs74107809 & A & G & 0.0697 \\
\hline 13 & rs9524741 & C & $\mathrm{T}$ & 0.1395 \\
\hline 13 & rs9524742 & $T$ & C & 0.1395 \\
\hline 13 & rs9524745 & A & G & 0.2326 \\
\hline 13 & rs2182264 & $\mathrm{T}$ & C & 0.2326 \\
\hline 13 & rs11619027 & A & G & 0.0697 \\
\hline 13 & rs9590154 & G & $\mathrm{T}$ & 0.2209 \\
\hline 13 & rs73553674 & $A$ & G & 0.2093 \\
\hline 13 & rs9590156 & A & G & 0.0697 \\
\hline 13 & rs9556451 & $\mathrm{T}$ & C & 0.0814 \\
\hline 13 & rs56996395 & A & AAT & 0.1744 \\
\hline 13 & rs9561760 & A & C & 0.407 \\
\hline 13 & rs111401227 & $\mathrm{T}$ & C & 0.4651 \\
\hline 13 & rs115036664 & A & G & 0.0581 \\
\hline 13 & rs114693747 & G & C & 0.0697 \\
\hline 13 & rs9524753 & G & C & 0.1512 \\
\hline 13 & rs12863006 & A & $\mathrm{T}$ & 0.186 \\
\hline 13 & rs60801806 & A & $\mathrm{T}$ & 0.1047 \\
\hline 13 & rs16950461 & C & $\mathrm{T}$ & 0.1047 \\
\hline 13 & rs73553699 & A & G & 0.0930 \\
\hline 13 & rs7318327 & $\mathrm{T}$ & C & 0.3953 \\
\hline 13 & rs144138469 & A & AAT & 0.1047 \\
\hline 13 & rs111776216 & A & C & 0.0930 \\
\hline 13 & rs114211115 & C & $\mathrm{T}$ & 0.1047 \\
\hline 13 & rs114979997 & C & $\mathrm{T}$ & 0.1047 \\
\hline 13 & rs74107818 & G & A & 0.1047 \\
\hline 13 & rs61043527 & $\mathrm{T}$ & TAA & 0.2442 \\
\hline 13 & rs9590160 & A & C & 0.0930 \\
\hline 13 & rs141851435 & G & C & 0.1047 \\
\hline
\end{tabular}

Table 1-A1 continues on the next page $\rightarrow$ 
Table 1-A1 (Continues...): Minor allele frequencies for 1846 polymorphisms in 43 black South Africans.

\begin{tabular}{|c|c|c|c|c|}
\hline Chromosome & Polymorphism & Minor allele & Major allele & $\begin{array}{l}\text { Minor allele } \\
\text { frequency }\end{array}$ \\
\hline 13 & rs9516518 & C & $\mathrm{T}$ & 0.1163 \\
\hline 13 & rs61530740 & G & A & 0.3372 \\
\hline 13 & rs9590161 & G & A & 0.1047 \\
\hline 13 & rs3770 & $A$ & G & 0.4302 \\
\hline 13 & rs 16950472 & G & A & 0.1047 \\
\hline 13 & rs9584273 & $\mathrm{T}$ & C & 0.0697 \\
\hline 13 & rs1059751 & G & A & 0.2442 \\
\hline 13 & rs4148553 & $T$ & C & 0.2442 \\
\hline 13 & rs74107819 & A & C & 0.1047 \\
\hline 13 & rs115226179 & $\mathrm{T}$ & C & 0.0581 \\
\hline 13 & rs4148551 & $T$ & C & 0.3605 \\
\hline 13 & rs3742106 & C & A & 0.3372 \\
\hline 13 & rs4148549 & C & $\mathrm{T}$ & 0.3605 \\
\hline 13 & rs115345973 & $\mathrm{T}$ & C & 0.0581 \\
\hline 13 & rs59600142 & A & G & 0.2791 \\
\hline 13 & rs61167065 & $\mathrm{T}$ & C & 0.2326 \\
\hline 13 & rs7331508 & G & A & 0.186 \\
\hline 13 & rs112975730 & C & G & 0.1977 \\
\hline 13 & rs200689258 & $A C$ & A & 0.0930 \\
\hline 13 & rs4773838 & $\mathrm{C}$ & A & 0.4651 \\
\hline 13 & rs4771904 & $T$ & G & 0.2674 \\
\hline 13 & rs9805226 & C & $\mathrm{T}$ & 0.1047 \\
\hline 13 & rs9302039 & T & A & 0.4884 \\
\hline 13 & rs114719300 & C & $\mathrm{T}$ & 0.0581 \\
\hline 13 & rs75080423 & C & A & 0.2907 \\
\hline 13 & rs202160354 & $\mathrm{CCA}$ & C & 0.1047 \\
\hline 13 & rs4148547 & $\mathrm{T}$ & C & 0.2907 \\
\hline 13 & rs4148546 & G & A & 0.3605 \\
\hline 13 & rs4274307 & C & $\mathrm{T}$ & 0.0697 \\
\hline 13 & rs4148544 & $\mathrm{T}$ & C & 0.2907 \\
\hline 13 & rs4148543 & G & A & 0.314 \\
\hline 13 & rs61967163 & G & A & 0.3372 \\
\hline 13 & rs67308757 & G & GA & 0.3372 \\
\hline 13 & rs191458655 & A & G & 0.0581 \\
\hline 13 & rs9561765 & A & G & 0.0814 \\
\hline 13 & rs116336902 & A & G & 0.0697 \\
\hline 13 & rs150571031 & C & CAT & 0.0697 \\
\hline 13 & rs7331488 & C & $\mathrm{T}$ & 0.0581 \\
\hline 13 & rs9302040 & A & C & 0.2558 \\
\hline 13 & rs58286099 & C & $\mathrm{T}$ & 0.0814 \\
\hline 13 & rs112792420 & $T$ & A & 0.2558 \\
\hline 13 & rs11568695 & $\mathrm{T}$ & C & 0.1512 \\
\hline 13 & rs9556455 & A & G & 0.2558 \\
\hline 13 & rs9561768 & C & $\mathrm{T}$ & 0.2558 \\
\hline 13 & rs115100521_t3 & G & $\mathrm{T}$ & 0.0581 \\
\hline 13 & rs9590168 & G & C & 0.2558 \\
\hline 13 & rs9590169 & G & A & 0.2558 \\
\hline 13 & rs115850104 & G & $\mathrm{T}$ & 0.0814 \\
\hline 13 & rs9561769 & $A$ & G & 0.2558 \\
\hline 13 & rs200912629 & CT & C & 0.2558 \\
\hline 13 & rs139228772 & AT & A & 0.2558 \\
\hline 13 & rs113574255 & C & G & 0.0814 \\
\hline 13 & rs61967172 & $T$ & C & 0.2558 \\
\hline 13 & rs61967173 & $\mathrm{T}$ & C & 0.2558 \\
\hline 13 & rs114400105 & G & $\mathrm{T}$ & 0.0814 \\
\hline 13 & rs10219913 & C & $\mathrm{T}$ & 0.2558 \\
\hline 13 & rs9302043 & G & A & 0.2558 \\
\hline 13 & rs61178570 & AT & A & 0.2558 \\
\hline 13 & rs7324971 & A & G & 0.0814 \\
\hline 13 & rs199553697 & CAAAT & $\mathrm{C}$ & 0.2442 \\
\hline
\end{tabular}

Table 1-A1 continues in the next column $\rightarrow$
Table 1-A1 (Continues...): Minor allele frequencies for 1846 polymorphisms in 43 black South Africans.

\begin{tabular}{|c|c|c|c|c|}
\hline Chromosome & Polymorphism & Minor allele & Major allele & $\begin{array}{l}\text { Minor allele } \\
\text { frequency }\end{array}$ \\
\hline 13 & rs61967174 & $\mathrm{T}$ & $A$ & 0.2558 \\
\hline 13 & rs61967175 & $A$ & $\mathrm{~T}$ & 0.2674 \\
\hline 13 & rs7324602 & G & A & 0.0814 \\
\hline 13 & rs7324065 & $\mathrm{T}$ & C & 0.0814 \\
\hline 13 & rs1189449 & C & $\mathrm{T}$ & 0.4535 \\
\hline 13 & rs113423125 & $A$ & $\mathrm{~T}$ & 0.0814 \\
\hline 13 & rs9302044 & G & A & 0.1628 \\
\hline 13 & rs1189445 & $\mathrm{T}$ & C & 0.4186 \\
\hline 13 & rs1189444 & C & $\mathrm{T}$ & 0.2907 \\
\hline 13 & rs1614102 & $\mathrm{C}$ & $\mathrm{T}$ & 0.3837 \\
\hline 13 & rs9561773 & $\mathrm{T}$ & C & 0.3256 \\
\hline 13 & rs9561774 & $\mathrm{T}$ & C & 0.314 \\
\hline 13 & rs5805899 & AT & A & 0.1047 \\
\hline 13 & rs9561776 & G & A & 0.4535 \\
\hline 13 & rs3782945 & A & G & 0.3372 \\
\hline 13 & rs3782946 & C & $\mathrm{T}$ & 0.4419 \\
\hline 13 & rs11343244 & $\mathrm{T}$ & TC & 0.1047 \\
\hline 13 & rs931112 & C & A & 0.0814 \\
\hline 13 & rs931111 & G & A & 0.3372 \\
\hline 13 & rs922522 & G & A & 0.3372 \\
\hline 13 & rs931110 & $\mathrm{T}$ & C & 0.1047 \\
\hline 13 & rs56695310 & A & C & 0.0581 \\
\hline 13 & rs1751033 & A & C & 0.2326 \\
\hline 13 & rs1729747 & G & C & 0.3372 \\
\hline 13 & rs57270423 & C & $\mathrm{T}$ & 0.3372 \\
\hline 13 & rs9302045 & $A$ & G & 0.1047 \\
\hline 13 & rs11840606 & G & A & 0.0581 \\
\hline 13 & rs7997839 & G & A & 0.1395 \\
\hline 13 & rs7997756 & $\mathrm{T}$ & C & 0.0814 \\
\hline 13 & rs201401074 & CAG & C & 0.1047 \\
\hline 13 & rs9590171 & C & A & 0.1047 \\
\hline 13 & rs150112578 & TC & $\mathrm{T}$ & 0.0814 \\
\hline 13 & rs1678387 & $\mathrm{T}$ & C & 0.1163 \\
\hline 13 & rs1678409 & T & C & 0.1047 \\
\hline 13 & rs1189457 & C & G & 0.3256 \\
\hline 13 & rs1189455 & A & G & 0.1047 \\
\hline 13 & rs112582196 & A & AG & 0.1047 \\
\hline 13 & rs148638661 & A & $\mathrm{T}$ & 0.0697 \\
\hline 13 & rs1678392 & A & G & 0.186 \\
\hline 13 & rs9524789 & A & $\mathrm{T}$ & 0.2209 \\
\hline 13 & rs115810488 & G & A & 0.1047 \\
\hline 13 & rs1189461 & $\mathrm{T}$ & C & 0.3023 \\
\hline 13 & rs1189462 & C & $\mathrm{T}$ & 0.1977 \\
\hline 13 & rs9590172 & $\mathrm{T}$ & C & 0.1047 \\
\hline 13 & rs1189463 & $\mathrm{T}$ & C & 0.3023 \\
\hline 13 & rs1751040 & C & $\mathrm{T}$ & 0.3023 \\
\hline 13 & rs9590173 & C & $\mathrm{T}$ & 0.1047 \\
\hline 13 & rs9972066 & $\mathrm{T}$ & C & 0.1047 \\
\hline 13 & rs2274401 & C & $\mathrm{T}$ & 0.4535 \\
\hline 13 & rs1189464 & $\mathrm{T}$ & C & 0.1279 \\
\hline 13 & rs1189465 & $C$ & $\mathrm{~T}$ & 0.1047 \\
\hline 13 & rs4148534 & $C$ & $\mathrm{~T}$ & 0.4535 \\
\hline 13 & rs1189466 & A & G & 0.0814 \\
\hline 13 & rs1189467 & $\mathrm{T}$ & A & 0.1279 \\
\hline 13 & rs1189468 & A & C & 0.1628 \\
\hline 13 & rs1678339 & $\mathrm{T}$ & C & 0.1279 \\
\hline 13 & rs1751042 & $\mathrm{T}$ & G & 0.1279 \\
\hline 13 & rs1751043 & A & G & 0.0814 \\
\hline 13 & rs1751045 & A & G & 0.0814 \\
\hline 13 & rs1751046 & G & A & 0.3488 \\
\hline
\end{tabular}

Table 1-A1 continues on the next page $\rightarrow$ 
Table 1-A1 (Continues...): Minor allele frequencies for 1846 polymorphisms in 43 black South Africans.

\begin{tabular}{|c|c|c|c|c|}
\hline Chromosome & Polymorphism & Minor allele & Major allele & $\begin{array}{l}\text { Minor allele } \\
\text { frequency }\end{array}$ \\
\hline 13 & rs1617888 & $\mathrm{T}$ & G & 0.3488 \\
\hline 13 & rs943289 & A & G & 0.1047 \\
\hline 13 & rs73557775 & A & G & 0.0814 \\
\hline 13 & rs1751051 & $\mathrm{T}$ & A & 0.2791 \\
\hline 13 & rs2766478 & A & $\mathrm{T}$ & 0.2791 \\
\hline 13 & rs12867863 & A & C & 0.0697 \\
\hline 13 & rs7987858 & $\mathrm{T}$ & C & 0.3721 \\
\hline 13 & rs1751052 & G & A & 0.3721 \\
\hline 13 & rs1189440 & G & A & 0.1047 \\
\hline 13 & rs1189439 & C & $\mathrm{T}$ & 0.3721 \\
\hline 13 & rs1189438 & G & A & 0.3721 \\
\hline 13 & rs9590177 & $\mathrm{T}$ & $\mathrm{C}$ & 0.1744 \\
\hline 13 & rs1189436 & A & G & 0.1047 \\
\hline 13 & rs1189435 & C & $\mathrm{T}$ & 0.1047 \\
\hline 13 & rs4148530 & $\mathrm{T}$ & C & 0.0814 \\
\hline 13 & rs1189434 & A & G & 0.1047 \\
\hline 13 & rs34857509 & A & G & 0.0814 \\
\hline 13 & rs35904677 & A & AT & 0.1047 \\
\hline 13 & rs1189433 & $\mathrm{C}$ & $\mathrm{T}$ & 0.1047 \\
\hline 13 & rs60205363 & CA & C & 0.0814 \\
\hline 13 & rs149521078 & A & C & 0.0697 \\
\hline 13 & rs145337567 & $\mathrm{T}$ & $\mathrm{C}$ & 0.0697 \\
\hline 13 & rs1617785 & G & A & 0.2907 \\
\hline 13 & rs1729760 & A & G & 0.2907 \\
\hline 13 & rs4148527 & A & G & 0.0814 \\
\hline 13 & rs12584534 & $\mathrm{T}$ & C & 0.0697 \\
\hline 13 & rs9590184 & C & $\mathrm{T}$ & 0.1744 \\
\hline 13 & rs145277775 & A & G & 0.0697 \\
\hline 13 & rs112327943 & A & C & 0.0697 \\
\hline 13 & rs147385814 & C & $\mathrm{T}$ & 0.0697 \\
\hline 13 & rs4773840 & G & A & 0.2442 \\
\hline 13 & rs74105436 & G & A & 0.2558 \\
\hline 13 & rs 16950650 & $\mathrm{~T}$ & C & 0.1395 \\
\hline 13 & rs1564355 & G & A & 0.314 \\
\hline 13 & rs1564354 & A & C & 0.314 \\
\hline 13 & rs1564353 & C & A & 0.314 \\
\hline 13 & rs143351018 & $\mathrm{T}$ & C & 0.0697 \\
\hline 13 & rs1751069 & A & G & 0.0930 \\
\hline 13 & rs4148515 & $\mathrm{T}$ & G & 0.2907 \\
\hline 13 & rs4148512 & A & G & 0.3256 \\
\hline 13 & rs4148509 & $\mathrm{T}$ & C & 0.3023 \\
\hline 13 & rs16950656 & G & $\mathrm{T}$ & 0.0465 \\
\hline 13 & rs57927922 & A & G & 0.0581 \\
\hline 13 & rs3782958 & $\mathrm{C}$ & G & 0.3023 \\
\hline 13 & rs61965885 & $\mathrm{T}$ & C & 0.3023 \\
\hline 13 & rs114479588 & $\mathrm{T}$ & G & 0.0581 \\
\hline 13 & rs72643607 & A & G & 0.0581 \\
\hline 13 & rs4148506 & $\mathrm{T}$ & C & 0.4884 \\
\hline 13 & rs1471481 & G & A & 0.1163 \\
\hline 13 & rs114964035 & A & G & 0.0697 \\
\hline 13 & rs79341676 & C & $\mathrm{T}$ & 0.0814 \\
\hline 13 & rs1678396 & A & G & 0.1628 \\
\hline 13 & rs1750996 & A & G & 0.4186 \\
\hline 13 & rs1729764 & A & G & 0.4186 \\
\hline 13 & rs1038138 & C & $\mathrm{T}$ & 0.3488 \\
\hline 13 & rs56261894 & $\mathrm{C}$ & $\mathrm{T}$ & 0.1163 \\
\hline 13 & rs1750999 & $\mathrm{T}$ & $\mathrm{C}$ & 0.1512 \\
\hline 13 & rs7982526 & $\mathrm{T}$ & G & 0.0465 \\
\hline 13 & rs72643633 & $\mathrm{T}$ & C & 0.0697 \\
\hline 13 & rs1189458 & G & A & 0.407 \\
\hline
\end{tabular}

Table 1-A1 continues in the next column $\rightarrow$
Table 1-A1 (Continues...): Minor allele frequencies for 1846 polymorphisms in 43 black South Africans.

\begin{tabular}{|c|c|c|c|c|}
\hline Chromosome & Polymorphism & Minor allele & Major allele & $\begin{array}{l}\text { Minor allele } \\
\text { frequency }\end{array}$ \\
\hline 13 & rs6492767 & $\mathrm{T}$ & C & 0.2093 \\
\hline 13 & rs6492768 & G & $A$ & 0.2093 \\
\hline 13 & rs1751003 & $A$ & G & 0.1512 \\
\hline 13 & rs3864996 & $\mathrm{C}$ & $\mathrm{T}$ & 0.2093 \\
\hline 13 & rs12584917 & $\mathrm{T}$ & C & 0.0581 \\
\hline 13 & rs4148496 & C & $\mathrm{T}$ & 0.1744 \\
\hline 13 & rs2478461 & $\mathrm{T}$ & C & 0.1395 \\
\hline 13 & rs2009772 & C & $\mathrm{T}$ & 0.0581 \\
\hline 13 & rs 1073500 & A & C & 0.2209 \\
\hline 13 & rs1751005 & $\mathrm{T}$ & C & 0.1744 \\
\hline 13 & rs11568662 & G & A & 0.0930 \\
\hline 13 & rs55994917 & $\mathrm{C}$ & A & 0.0581 \\
\hline 13 & rs7330933 & G & A & 0.407 \\
\hline 13 & rs61965904 & $\mathrm{T}$ & C & 0.0581 \\
\hline 13 & rs2793821 & $\mathrm{T}$ & G & 0.2791 \\
\hline 13 & rs200790129 & $\mathrm{T}$ & A & 0.1395 \\
\hline 13 & rs4148493 & G & $\mathrm{T}$ & 0.4884 \\
\hline 13 & rs148748821 & $\mathrm{CTT}$ & C & 0.1395 \\
\hline 13 & rs118084803 & G & A & 0.0697 \\
\hline 13 & rs1887162 & $\mathrm{T}$ & G & 0.1628 \\
\hline 13 & rs150301651 & TCCA & $\mathrm{T}$ & 0.1047 \\
\hline 13 & rs4148488 & G & C & 0.0581 \\
\hline 13 & rs1564351 & G & A & 0.0581 \\
\hline 13 & rs3843689 & G & A & 0.1628 \\
\hline 13 & rs2766474 & A & G & 0.1977 \\
\hline 13 & rs2148529 & G & $\mathrm{T}$ & 0.0581 \\
\hline 13 & rs 1557070 & A & G & 0.2442 \\
\hline 13 & rs4773843 & $\mathrm{T}$ & C & 0.1047 \\
\hline 13 & rs2487566 & G & A & 0.4884 \\
\hline 13 & rs4148482 & $\mathrm{T}$ & $\mathrm{C}$ & 0.4535 \\
\hline 13 & rs1751015 & C & $\mathrm{T}$ & 0.1279 \\
\hline 13 & rs4148481 & A & G & 0.4767 \\
\hline 13 & rs3864997 & $\mathrm{T}$ & G & 0.4767 \\
\hline 13 & rs1678383 & G & $\mathrm{T}$ & 0.1163 \\
\hline 13 & rs1678384 & A & G & 0.2558 \\
\hline 13 & rs112922776 & $\mathrm{T}$ & C & 0.2209 \\
\hline 13 & rs9516530 & C & $\mathrm{T}$ & 0.3953 \\
\hline 13 & rs1678386 & C & A & 0.3837 \\
\hline 13 & rs1751021 & C & $\mathrm{T}$ & 0.4767 \\
\hline 13 & rs1678388 & G & A & 0.0581 \\
\hline 13 & rs1751027 & G & A & 0.2791 \\
\hline 13 & rs1751029 & A & G & 0.0581 \\
\hline 13 & rs1617844 & G & A & 0.1279 \\
\hline 13 & rs3818493 & G & A & 0.4419 \\
\hline 13 & rs2274405 & $\mathrm{T}$ & C & 0.4419 \\
\hline 13 & rs2274406 & C & $\mathrm{T}$ & 0.3023 \\
\hline 13 & rs2274407 & A & C & 0.2326 \\
\hline 13 & rs6413442 & $\mathrm{T}$ & A & 0.2209 \\
\hline 13 & rs3818495 & $\mathrm{T}$ & A & 0.3023 \\
\hline 13 & rs7331142 & C & $\mathrm{T}$ & 0.4302 \\
\hline 13 & rs899494 & A & G & 0.2907 \\
\hline 13 & rs1678403 & C & G & 0.3605 \\
\hline 13 & rs943288 & A & $\mathrm{T}$ & 0.1163 \\
\hline 13 & rs1678400 & A & G & 0.1163 \\
\hline 13 & rs114535776 & A & G & 0.186 \\
\hline 13 & rs9524828 & C & $\mathrm{T}$ & 0.4535 \\
\hline 13 & rs9516537 & G & A & 0.4535 \\
\hline 13 & rs9516538 & $\mathrm{T}$ & $C$ & 0.4535 \\
\hline 13 & rs115962025 & $\mathrm{T}$ & A & 0.0814 \\
\hline 13 & rs7332663 & G & A & 0.4535 \\
\hline
\end{tabular}

Table 1-A1 continues on the next page $\rightarrow$ 
Table 1-A1 (Continues...): Minor allele frequencies for 1846 polymorphisms in 43 black South Africans.

\begin{tabular}{|c|c|c|c|c|}
\hline Chromosome & Polymorphism & Minor allele & Major allele & $\begin{array}{l}\text { Minor allele } \\
\text { frequency }\end{array}$ \\
\hline 13 & rs7332836 & G & A & 0.4535 \\
\hline 13 & rs7333281 & G & $\mathrm{T}$ & 0.2674 \\
\hline 13 & rs7335147 & $\mathrm{T}$ & C & 0.5 \\
\hline 13 & rs9516539 & A & G & 0.5 \\
\hline 13 & rs9524830 & A & $\mathrm{T}$ & 0.5 \\
\hline 13 & rs7322825 & C & $\mathrm{T}$ & 0.5 \\
\hline 13 & rs7139533 & A & G & 0.5 \\
\hline 13 & rs35475476 & $\mathrm{T}$ & TG & 0.1163 \\
\hline 13 & rs9524831 & A & $\mathrm{C}$ & 0.5 \\
\hline 13 & rs7983336 & G & A & 0.5 \\
\hline 13 & rs7987653 & $\mathrm{T}$ & C & 0.5 \\
\hline 13 & rs9524833 & A & G & 0.5 \\
\hline 13 & rs4334136 & C & A & 0.5 \\
\hline 13 & rs58721524 & A & C & 0.0465 \\
\hline 13 & rs1824913 & G & A & 0.5 \\
\hline 13 & rs1824912 & A & G & 0.5 \\
\hline 13 & rs1824911 & $\mathrm{T}$ & A & 0.5 \\
\hline 13 & rs7999175 & C & $\mathrm{T}$ & 0.5 \\
\hline 13 & rs143604864 & C & $\mathrm{T}$ & 0.186 \\
\hline 13 & rs9524835 & A & G & 0.5 \\
\hline 13 & rs9524836 & A & G & 0.5 \\
\hline 13 & rs4773847 & $\mathrm{T}$ & $\mathrm{C}$ & 0.5 \\
\hline 13 & rs201259935 & C & CA & 0.186 \\
\hline 13 & rs4148467 & $\mathrm{T}$ & C & 0.5 \\
\hline 13 & rs34429583 & $\mathrm{AC}$ & A & 0.4535 \\
\hline 13 & rs34429583_t3 & C & A & 0.4535 \\
\hline 13 & rs4771907 & C & G & 0.5 \\
\hline 13 & rs4148465 & A & G & 0.5 \\
\hline 13 & rs115789275 & G & C & 0.186 \\
\hline 13 & rs4148464 & G & A & 0.5 \\
\hline 13 & rs4148463 & C & G & 0.5 \\
\hline 13 & rs7321744 & G & A & 0.5 \\
\hline 13 & rs113500636 & CCACTG & C & 0.5 \\
\hline 13 & rs6492769 & $\mathrm{T}$ & C & 0.5 \\
\hline 13 & rs75557542 & G & A & 0.5 \\
\hline 13 & rs114513462 & $\mathrm{T}$ & C & 0.0814 \\
\hline 13 & rs9524840 & A & G & 0.5 \\
\hline 13 & rs115690139 & G & A & 0.186 \\
\hline 13 & rs9590203 & C & $\mathrm{T}$ & 0.0697 \\
\hline 13 & rs4111022 & $\mathrm{T}$ & C & 0.4535 \\
\hline 13 & rs7321623 & A & G & 0.5 \\
\hline 13 & rs11431283 & CT & C & 0.5 \\
\hline 13 & rs4771908 & C & $\mathrm{T}$ & 0.5 \\
\hline 13 & rs67413584 & A & AATATT & 0.5 \\
\hline 13 & rs2766476 & A & C & 0.1163 \\
\hline 13 & rs9302048 & G & C & 0.2674 \\
\hline 13 & rs4773848 & A & G & 0.3721 \\
\hline 13 & rs9590204 & $\mathrm{T}$ & G & 0.2674 \\
\hline 13 & rs9590205 & $\mathrm{T}$ & A & 0.2674 \\
\hline 13 & rs9302049 & $\mathrm{C}$ & $\mathrm{T}$ & 0.2674 \\
\hline 13 & rs79083026 & G & C & 0.0697 \\
\hline 13 & rs114579713 & $\mathrm{T}$ & C & 0.186 \\
\hline 13 & rs4773849 & G & A & 0.3721 \\
\hline 13 & rs1926656 & G & A & 0.3953 \\
\hline 13 & rs1926657 & $\mathrm{T}$ & C & 0.3372 \\
\hline 13 & rs7330519 & C & $\mathrm{T}$ & 0.3837 \\
\hline 13 & rs7325019 & C & $\mathrm{T}$ & 0.3488 \\
\hline 13 & rs4612933 & $\mathrm{T}$ & C & 0.3488 \\
\hline 13 & rs200179424 & A & $\mathrm{ACT}$ & 0.3488 \\
\hline 13 & rs4371040 & $\mathrm{T}$ & G & 0.3488 \\
\hline
\end{tabular}

Table 1-A1 continues in the next column $\rightarrow$
Table 1-A1 (Continues...): Minor allele frequencies for 1846 polymorphisms in 43 black South Africans.

\begin{tabular}{|c|c|c|c|c|}
\hline Chromosome & Polymorphism & Minor allele & Major allele & $\begin{array}{l}\text { Minor allele } \\
\text { frequency }\end{array}$ \\
\hline 13 & rs4303338 & $\mathrm{C}$ & G & 0.3488 \\
\hline 13 & rs9516545 & G & A & 0.3488 \\
\hline 13 & rs2892707 & $\mathrm{T}$ & C & 0.3488 \\
\hline 13 & rs7331366 & $\mathrm{T}$ & C & 0.1279 \\
\hline 13 & rs4773850 & G & $\mathrm{T}$ & 0.3837 \\
\hline 13 & rs7330776 & A & G & 0.0814 \\
\hline 13 & rs150285358 & G & C & 0.0697 \\
\hline 13 & rs114827818 & G & A & 0.0581 \\
\hline 13 & rs141735320 & A & G & 0.0697 \\
\hline 13 & rs12428470 & $\mathrm{C}$ & $\mathrm{T}$ & 0.3488 \\
\hline 13 & rs116263184 & A & $\mathrm{T}$ & 0.0697 \\
\hline 13 & rs12854605 & G & $\mathrm{T}$ & 0.3488 \\
\hline 13 & rs8001451 & G & $\mathrm{T}$ & 0.0697 \\
\hline 13 & rs58296130 & G & A & 0.186 \\
\hline 13 & rs9524845 & $\mathrm{T}$ & C & 0.3488 \\
\hline 13 & rs4636781 & G & A & 0.3488 \\
\hline 13 & rs4505186 & G & A & 0.3488 \\
\hline 13 & rs4520712 & A & G & 0.3488 \\
\hline 13 & rs4773853 & G & A & 0.3488 \\
\hline 13 & rs4773854 & G & A & 0.3488 \\
\hline 13 & rs4773855 & A & C & 0.3488 \\
\hline 13 & rs6492771 & $C$ & $\mathrm{~T}$ & 0.3488 \\
\hline 13 & rs9590206 & $\mathrm{T}$ & C & 0.0697 \\
\hline 13 & rs59815481 & A & G & 0.0581 \\
\hline 13 & rs7326711 & A & G & 0.3953 \\
\hline 13 & rs61972720 & G & A & 0.1163 \\
\hline 13 & rs16950823 & G & A & 0.186 \\
\hline 13 & rs4148459 & C & $\mathrm{T}$ & 0.1163 \\
\hline 13 & rs4148456 & C & $\mathrm{T}$ & 0.1163 \\
\hline 13 & rs4148455 & $\mathrm{T}$ & C & 0.2326 \\
\hline 13 & rs9590207 & A & G & 0.0930 \\
\hline 13 & rs35347010 & C & СCTT & 0.0930 \\
\hline 13 & rs114592667 & $\mathrm{T}$ & C & 0.0814 \\
\hline 13 & rs77037338 & A & G & 0.1163 \\
\hline 13 & rs61972725 & A & $\mathrm{T}$ & 0.1163 \\
\hline 13 & rs2892713 & $\mathrm{T}$ & C & 0.1163 \\
\hline 13 & rs7337077 & $\mathrm{T}$ & C & 0.0581 \\
\hline 13 & rs7329532 & A & G & 0.0581 \\
\hline 13 & rs7336954 & C & $\mathrm{T}$ & 0.0581 \\
\hline 13 & rs9590209 & A & C & 0.0697 \\
\hline 13 & rs9524859 & $\mathrm{T}$ & C & 0.3372 \\
\hline 13 & rs9524860 & G & $\mathrm{T}$ & 0.3372 \\
\hline 13 & rs4479103 & $\mathrm{C}$ & $\mathrm{T}$ & 0.2907 \\
\hline 13 & rs4283094 & C & G & 0.2907 \\
\hline 13 & rs56608089 & $\mathrm{T}$ & C & 0.2907 \\
\hline 13 & rs9524862 & G & A & 0.2907 \\
\hline 13 & rs9302051 & G & A & 0.2907 \\
\hline 13 & rs9516547 & C & $\mathrm{T}$ & 0.0697 \\
\hline 13 & rs4148452 & A & G & 0.2907 \\
\hline 13 & rs4148451 & G & C & 0.2907 \\
\hline 13 & rs4148450 & C & $\mathrm{T}$ & 0.0697 \\
\hline 13 & rs8003006 & G & A & 0.2907 \\
\hline 13 & rs149464244 & C & $\mathrm{T}$ & 0.0465 \\
\hline 13 & rs6492772 & $\mathrm{T}$ & C & 0.0697 \\
\hline 13 & rs10508018 & A & G & 0.1279 \\
\hline 13 & rs7324464 & A & G & 0.0697 \\
\hline 13 & rs7329555 & $\mathrm{T}$ & G & 0.2907 \\
\hline 13 & rs4771909 & $\mathrm{T}$ & C & 0.2209 \\
\hline 13 & rs4148445 & C & $\mathrm{T}$ & 0.0814 \\
\hline 13 & rs4148443 & C & $\mathrm{T}$ & 0.2907 \\
\hline
\end{tabular}

Table 1-A1 continues on the next page $\rightarrow$ 
Table 1-A1 (Continues...): Minor allele frequencies for 1846 polymorphisms in 43 black South Africans.

\begin{tabular}{|c|c|c|c|c|}
\hline Chromosome & Polymorphism & Minor allele & Major allele & $\begin{array}{l}\text { Minor allele } \\
\text { frequency }\end{array}$ \\
\hline 13 & rs4148442 & $T$ & $\mathrm{C}$ & 0.2558 \\
\hline 13 & rs36077567 & GA & G & 0.2093 \\
\hline 13 & rs4148441 & A & G & 0.0697 \\
\hline 13 & rs4148440 & $\mathrm{T}$ & C & 0.0814 \\
\hline 13 & rs7335275 & C & $\mathrm{T}$ & 0.1163 \\
\hline 13 & rs7336202 & $A$ & $\mathrm{~T}$ & 0.1163 \\
\hline 13 & rs8000333 & A & G & 0.1163 \\
\hline 13 & rs9524864 & $\mathrm{T}$ & C & 0.0814 \\
\hline 13 & rs7982955 & A & G & 0.1163 \\
\hline 13 & rs4773861 & $\mathrm{T}$ & C & 0.1163 \\
\hline 13 & rs60115298 & C & CT & 0.1163 \\
\hline 13 & rs12584649 & C & $\mathrm{T}$ & 0.3837 \\
\hline 13 & rs9524866 & G & A & 0.1163 \\
\hline 13 & rs60769464 & A & C & 0.0465 \\
\hline 13 & rs7320383 & G & A & 0.1163 \\
\hline 13 & rs9516550 & G & C & 0.1512 \\
\hline 13 & rs735468891 & $A$ & G & 0.0465 \\
\hline 13 & rs9556467 & C & $\mathrm{T}$ & 0.1163 \\
\hline 13 & rs2389227 & $T$ & C & 0.1163 \\
\hline 13 & rs2389228 & G & $A$ & 0.1279 \\
\hline 13 & rs7319126 & A & C & 0.2558 \\
\hline 13 & rs74893509 & C & $\mathrm{T}$ & 0.2209 \\
\hline 13 & rs76655052 & $\mathrm{T}$ & G & 0.2209 \\
\hline 13 & rs7320375 & G & $A$ & 0.2674 \\
\hline 13 & rs7325256 & C & $\mathrm{T}$ & 0.2674 \\
\hline 13 & rs7325861 & G & $\mathrm{T}$ & 0.2674 \\
\hline 13 & rs4148433 & C & $\mathrm{T}$ & 0.2093 \\
\hline 13 & rs4148432 & $\mathrm{T}$ & C & 0.2674 \\
\hline 13 & rs4148430 & A & $\mathrm{T}$ & 0.2674 \\
\hline 13 & rs16950847 & $\mathrm{T}$ & A & 0.2093 \\
\hline 13 & rs79474727 & A & G & 0.2093 \\
\hline 13 & rs9524869 & C & G & 0.3605 \\
\hline 13 & rs11842634 & C & $\mathrm{T}$ & 0.2674 \\
\hline 13 & rs201251479 & $A C$ & A & 0.1163 \\
\hline 13 & rs59689275 & C & A & 0.1163 \\
\hline 13 & rs11843001 & C & $\mathrm{T}$ & 0.2558 \\
\hline 13 & rs7338004 & A & G & 0.0581 \\
\hline 13 & rs7338429 & A & G & 0.0581 \\
\hline 13 & rs7321532 & $\mathrm{T}$ & C & 0.0581 \\
\hline 13 & rs11843102 & G & A & 0.0581 \\
\hline 13 & rs7986087 & $\mathrm{T}$ & C & 0.2558 \\
\hline 13 & rs7987353 & G & A & 0.2558 \\
\hline 13 & rs60223404 & $\mathrm{T}$ & G & 0.0581 \\
\hline 13 & rs9516552 & C & $\mathrm{T}$ & 0.0814 \\
\hline 13 & rs79921462 & C & $\mathrm{T}$ & 0.0930 \\
\hline 13 & rs870004 & A & G & 0.3721 \\
\hline 13 & rs7333118 & T & G & 0.0465 \\
\hline 13 & rs9590228 & C & $\mathrm{T}$ & 0.3837 \\
\hline 13 & rs9590229 & C & $\mathrm{T}$ & 0.3837 \\
\hline 13 & rs7317112 & A & G & 0.3837 \\
\hline 13 & rs7322318 & T & C & 0.3372 \\
\hline 13 & rs73548830 & $\mathrm{T}$ & C & 0.0465 \\
\hline 13 & rs150988051 & ACATTGC & A & 0.1163 \\
\hline 13 & rs73548833 & C & A & 0.0465 \\
\hline 13 & rs7324503 & C & A & 0.1163 \\
\hline 13 & rs201187127 & AAAAAT & A & 0.0465 \\
\hline 13 & rs112859374 & $\mathrm{T}$ & C & 0.0465 \\
\hline 13 & rs56891151 & G & A & 0.0465 \\
\hline 13 & rs59514866 & G & C & 0.0465 \\
\hline 13 & rs4148428 & C & $\mathrm{T}$ & 0.2093 \\
\hline
\end{tabular}

Table 1-A1 continues in the next column $\rightarrow$
Table 1-A1 (Continues...): Minor allele frequencies for 1846 polymorphisms in 43 black South Africans.

\begin{tabular}{|c|c|c|c|c|}
\hline Chromosome & Polymorphism & Minor allele & Major allele & $\begin{array}{l}\text { Minor allele } \\
\text { frequency }\end{array}$ \\
\hline 13 & rs76888097 & $\mathrm{T}$ & C & 0.0930 \\
\hline 13 & rs9524873 & $A$ & G & 0.1395 \\
\hline 13 & rs9590231 & $A$ & G & 0.0814 \\
\hline 13 & rs4773866 & $\mathrm{T}$ & C & 0.1279 \\
\hline 13 & rs144363729 & $\mathrm{T}$ & C & 0.0581 \\
\hline 13 & rs9524879 & $\mathrm{T}$ & C & 0.3721 \\
\hline 13 & rs73548889 & C & $T$ & 0.0581 \\
\hline 13 & rs7986283 & G & A & 0.1512 \\
\hline 13 & rs7328426 & $\mathrm{T}$ & C & 0.3605 \\
\hline 13 & rs146331560 & A & G & 0.0814 \\
\hline 13 & rs9561830 & $A$ & G & 0.2558 \\
\hline 13 & rs147524788 & G & A & 0.1047 \\
\hline 13 & rs7330673 & G & $\mathrm{T}$ & 0.2558 \\
\hline 13 & rs7982930 & $\mathrm{T}$ & C & 0.0814 \\
\hline 13 & rs871051 & $\mathrm{T}$ & G & 0.407 \\
\hline 13 & rs8001444 & C & $\mathrm{T}$ & 0.1744 \\
\hline 13 & rs6650325 & A & C & 0.1744 \\
\hline 13 & rs9524896 & A & G & 0.1628 \\
\hline 13 & rs61974974 & A & G & 0.1744 \\
\hline 13 & rs869951 & G & C & 0.1744 \\
\hline 13 & rs2993579 & $\mathrm{T}$ & C & 0.1744 \\
\hline 13 & rs868853 & $\mathrm{C}$ & $\mathrm{T}$ & 0.2674 \\
\hline 13 & rs1764419 & A & G & 0.1744 \\
\hline 13 & rs1764417 & $\mathrm{T}$ & G & 0.186 \\
\hline 13 & rs9561837 & $\mathrm{T}$ & C & 0.3837 \\
\hline 13 & rs2992904 & C & A & 0.314 \\
\hline 13 & rs2993582 & $\mathrm{T}$ & G & 0.314 \\
\hline 13 & rs2992905 & $\mathrm{T}$ & C & 0.314 \\
\hline 13 & rs9302056 & T & A & 0.314 \\
\hline 13 & rs9302057 & G & A & 0.0697 \\
\hline 13 & rs9524899 & A & G & 0.3837 \\
\hline 13 & rs2993583 & C & A & 0.314 \\
\hline 13 & rs2992907 & $T$ & C & 0.2791 \\
\hline 13 & rs2992908 & A & G & 0.314 \\
\hline 13 & rs200012169 & A & AAG & 0.314 \\
\hline 13 & rs147591451 & A & AG & 0.314 \\
\hline 13 & rs2992909 & $\mathrm{T}$ & C & 0.314 \\
\hline 13 & rs7332635 & A & G & 0.3837 \\
\hline 13 & rs2993584 & A & C & 0.2791 \\
\hline 13 & rs2993585 & A & G & 0.314 \\
\hline 13 & rs9524901 & C & A & 0.3837 \\
\hline 13 & rs2992910 & A & $\mathrm{T}$ & 0.3256 \\
\hline 13 & rs2993586 & C & $\mathrm{T}$ & 0.2791 \\
\hline 13 & rs1764410 & $\mathrm{T}$ & C & 0.1628 \\
\hline 13 & rs7328570 & A & G & 0.1395 \\
\hline 13 & rs2993590 & C & $\mathrm{T}$ & 0.1395 \\
\hline 13 & rs7983929 & C & $\mathrm{T}$ & 0.4767 \\
\hline 13 & rs8000617 & $\mathrm{T}$ & C & 0.4767 \\
\hline 13 & rs7984107 & A & $T$ & 0.4767 \\
\hline 13 & rs142604921 & $\mathrm{T}$ & TG & 0.0581 \\
\hline 13 & rs9302061 & T & C & 0.4767 \\
\hline 13 & rs9302064 & $\mathrm{C}$ & A & 0.4884 \\
\hline 13 & rs4773880 & $\mathrm{T}$ & C & 0.0581 \\
\hline 13 & rs7995750 & A & $\mathrm{T}$ & 0.4767 \\
\hline 13 & rs4773882 & A & G & 0.0581 \\
\hline 13 & rs28843704 & $\mathrm{T}$ & C & 0.0697 \\
\hline 13 & rs7337939 & C & $\mathrm{T}$ & 0.2326 \\
\hline 13 & rs66774953 & G & $\mathrm{T}$ & 0.2326 \\
\hline 13 & rs4773884 & G & A & 0.4419 \\
\hline 13 & rs193003058 & A & G & 0.0814 \\
\hline
\end{tabular}

Table 1-A1 continues on the next page $\rightarrow$ 
Table 1-A1 (Continues...): Minor allele frequencies for 1846 polymorphisms in 43 black South Africans.

\begin{tabular}{|c|c|c|c|c|}
\hline Chromosome & Polymorphism & Minor allele & Major allele & $\begin{array}{l}\text { Minor allele } \\
\text { frequency }\end{array}$ \\
\hline 13 & rs9524910 & G & A & 0.2674 \\
\hline 13 & rs4773885 & $\mathrm{G}$ & A & 0.4767 \\
\hline 13 & rs17189568 & C & $\mathrm{T}$ & 0.1163 \\
\hline 13 & rs9590237 & A & G & 0.0697 \\
\hline 13 & rs9524920 & $A$ & G & 0.3488 \\
\hline 13 & rs60338761 & C & $\mathrm{T}$ & 0.1047 \\
\hline 13 & rs 2484983 & G & A & 0.2093 \\
\hline 13 & rs2993600 & G & $\mathrm{T}$ & 0.407 \\
\hline 13 & rs73553102 & C & $\mathrm{T}$ & 0.1163 \\
\hline 13 & rs8001940 & $A$ & C & 0.2558 \\
\hline 13 & rs9524925 & G & $A$ & 0.1744 \\
\hline 13 & rs1766893 & G & A & 0.1047 \\
\hline 13 & rs2993604 & C & G & 0.1977 \\
\hline 13 & rs2993605 & A & G & 0.1977 \\
\hline 13 & rs2993606 & A & G & 0.1977 \\
\hline 13 & rs77146956 & C & $\mathrm{T}$ & 0.1977 \\
\hline 13 & rs71432038 & C & $\mathrm{T}$ & 0.1977 \\
\hline 13 & rs66489010 & G & A & 0.1977 \\
\hline 13 & rs 28671610 & C & $\mathrm{T}$ & 0.1977 \\
\hline 13 & rs16950985 & $\mathrm{T}$ & G & 0.1977 \\
\hline 13 & rs2389257 & A & C & 0.1977 \\
\hline 13 & rs2389256 & G & A & 0.1977 \\
\hline 13 & rs1764430 & G & C & 0.1977 \\
\hline 13 & rs1766912 & G & A & 0.186 \\
\hline 13 & rs1766911 & G & A & 0.186 \\
\hline 13 & rs2389254 & G & A & 0.1977 \\
\hline 13 & rs61478273 & CAG & C & 0.1977 \\
\hline 13 & rs2485972 & A & G & 0.1977 \\
\hline 13 & rs 12875123 & A & $\mathrm{T}$ & 0.1977 \\
\hline 13 & rs2993554 & G & A & 0.1977 \\
\hline 13 & rs2389253 & C & $\mathrm{T}$ & 0.1977 \\
\hline 13 & rs116182952 & G & $\mathrm{T}$ & 0.1628 \\
\hline 13 & rs2992886 & A & G & 0.2093 \\
\hline 13 & rs2993555 & $\mathrm{T}$ & C & 0.2093 \\
\hline 13 & rs2992887 & C & A & 0.2093 \\
\hline 13 & rs114675293 & $\mathrm{T}$ & A & 0.1628 \\
\hline 13 & rs2992888 & G & A & 0.1977 \\
\hline 13 & rs1620972 & A & G & 0.1977 \\
\hline 13 & rs149181983 & AT & A & 0.1628 \\
\hline 13 & rs11619347 & A & G & 0.1628 \\
\hline 13 & rs 10508015 & C & $\mathrm{T}$ & 0.2209 \\
\hline 13 & rs12584768 & C & $\mathrm{T}$ & 0.1977 \\
\hline 13 & rs9524936 & G & C & 0.1977 \\
\hline 13 & rs9524937 & G & $\mathrm{T}$ & 0.1977 \\
\hline 13 & rs9516568 & G & A & 0.4535 \\
\hline 13 & rs9516572 & $\mathrm{T}$ & $\mathrm{C}$ & 0.1977 \\
\hline 19 & rs200039365 & G & $\mathrm{GC}$ & 0.1047 \\
\hline 19 & rs60533951 & A & G & 0.1047 \\
\hline 19 & rs7937 & $T$ & C & 0.2326 \\
\hline 19 & rs76268776 & $T$ & C & 0.0581 \\
\hline 19 & rs 2545761 & $T$ & C & 0.1279 \\
\hline 19 & rs 2545763 & A & G & 0.0930 \\
\hline 19 & rs79692712 & G & C & 0.0581 \\
\hline 19 & rs11666504 & $T$ & C & 0.0930 \\
\hline 19 & rs 16974537 & $A$ & G & 0.0465 \\
\hline 19 & rs10405596 & $\mathrm{T}$ & C & 0.2558 \\
\hline 19 & rs 2545769 & G & A & 0.4767 \\
\hline 19 & rs2545771 & A & G & 0.0930 \\
\hline 19 & rs 2545772 & A & G & 0.0814 \\
\hline 19 & rs11879672 & $\mathrm{T}$ & $C$ & 0.4767 \\
\hline
\end{tabular}

Table 1-A1 continues in the next column $\rightarrow$
Table 1-A1 (Continues...): Minor allele frequencies for 1846 polymorphisms in 43 black South Africans.

\begin{tabular}{|c|c|c|c|c|}
\hline Chromosome & Polymorphism & Minor allele & Major allele & $\begin{array}{l}\text { Minor allele } \\
\text { frequency }\end{array}$ \\
\hline 19 & rs28483485 & $\mathrm{T}$ & C & 0.4767 \\
\hline 19 & rs112673025 & $A$ & G & 0.0465 \\
\hline 19 & rs79366653 & G & A & 0.0581 \\
\hline 19 & rs11878604 & C & $\mathrm{T}$ & 0.2907 \\
\hline 19 & rs55978439 & A & $\mathrm{T}$ & 0.0465 \\
\hline 19 & rs2258314 & $\mathrm{T}$ & C & 0.0697 \\
\hline 19 & rs10853742 & G & C & 0.1977 \\
\hline 19 & rs12327581 & $\mathrm{T}$ & C & 0.4419 \\
\hline 19 & rs11667314 & $\mathrm{T}$ & C & 0.1977 \\
\hline 19 & rs7251418 & A & G & 0.1047 \\
\hline 19 & rs76112798 & $\mathrm{T}$ & C & 0.0697 \\
\hline 19 & rs7248240 & G & C & 0.1163 \\
\hline 19 & rs56164728 & C & $\mathrm{T}$ & 0.0697 \\
\hline 19 & rs28399462 & A & G & 0.0697 \\
\hline 19 & rs4803380 & $\mathrm{T}$ & C & 0.0697 \\
\hline 19 & rs28399454 & $\mathrm{T}$ & C & 0.0697 \\
\hline 19 & rs144437384 & A & G & 0.0697 \\
\hline 19 & rs72549444 & A & G & 0.0697 \\
\hline 19 & rs56113850 & C & $\mathrm{T}$ & 0.2791 \\
\hline 19 & rs28399433 & C & A & 0.0697 \\
\hline 19 & rs61663607 & $C$ & $\mathrm{~T}$ & 0.1512 \\
\hline 19 & rs111822043 & A & $\mathrm{T}$ & 0.0465 \\
\hline 19 & rs8102683 & $\mathrm{T}$ & C & 0.2209 \\
\hline 19 & rs8105704 & $\mathrm{T}$ & C & 0.2093 \\
\hline 19 & rs12610432 & $\mathrm{T}$ & C & 0.2093 \\
\hline 19 & rs186830274 & C & $\mathrm{T}$ & 0.0581 \\
\hline 19 & rs111867898 & C & $\mathrm{T}$ & 0.0465 \\
\hline 19 & rs4570983 & $\mathrm{T}$ & C & 0.1279 \\
\hline 19 & rs75152309 & A & $\mathrm{T}$ & 0.0697 \\
\hline 19 & rs74493998 & $\mathrm{T}$ & C & 0.0697 \\
\hline 19 & rs28575771 & A & G & 0.2209 \\
\hline 19 & rs2261144 & G & A & 0.1512 \\
\hline 19 & rs12975382 & $\mathrm{T}$ & G & 0.4535 \\
\hline 19 & rs73032311 & C & $\mathrm{T}$ & 0.0697 \\
\hline 19 & rs73032316 & C & G & 0.0697 \\
\hline 19 & rs3815706 & G & $\mathrm{T}$ & 0.0697 \\
\hline 19 & rs56081734 & A & C & 0.4535 \\
\hline 19 & rs66882672 & G & A & 0.0814 \\
\hline 19 & rs67808403 & G & A & 0.0814 \\
\hline 19 & rs149560129 & G & A & 0.0465 \\
\hline 19 & rs4803393 & C & $\mathrm{T}$ & 0.1163 \\
\hline 19 & rs79809963 & C & A & 0.0814 \\
\hline 19 & rs76734307 & C & $\mathrm{T}$ & 0.0814 \\
\hline 19 & rs10853743 & $\mathrm{T}$ & C & 0.0697 \\
\hline 19 & rs3875155 & C & $\mathrm{T}$ & 0.0814 \\
\hline 19 & rs4105141 & A & $\mathrm{T}$ & 0.2326 \\
\hline 19 & rs5007415 & A & C & 0.2326 \\
\hline 19 & rs10411264 & $\mathrm{T}$ & C & 0.2326 \\
\hline 19 & rs115564457 & $\mathrm{T}$ & C & 0.1279 \\
\hline 19 & rs28472879 & A & G & 0.2326 \\
\hline 19 & rs28463685 & A & G & 0.1395 \\
\hline 19 & rs10406188 & G & A & 0.2326 \\
\hline 19 & rs8103288 & G & C & 0.2326 \\
\hline 19 & rs8103444 & C & A & 0.0697 \\
\hline 19 & rs10414481 & $\mathrm{T}$ & C & 0.2326 \\
\hline 19 & rs78374326 & G & $\mathrm{T}$ & 0.2326 \\
\hline 19 & rs3865457 & $\mathrm{T}$ & C & 0.2326 \\
\hline 19 & rs12611183 & A & $\mathrm{T}$ & 0.0814 \\
\hline 19 & rs199970591 & $\mathrm{T}$ & TATCA & 0.0814 \\
\hline 19 & rs76297159 & G & A & 0.0814 \\
\hline
\end{tabular}

Table 1-A1 continues on the next page $\rightarrow$ 
Table 1-A1 (Continues...): Minor allele frequencies for 1846 polymorphisms in 43 black South Africans.

\begin{tabular}{|c|c|c|c|c|}
\hline Chromosome & Polymorphism & Minor allele & Major allele & $\begin{array}{l}\text { Minor allele } \\
\text { frequency }\end{array}$ \\
\hline 19 & rs12609982 & G & A & 0.0814 \\
\hline 19 & rs 12608615 & C & $\mathrm{T}$ & 0.0814 \\
\hline 19 & rs34127861 & TA & $\mathrm{T}$ & 0.0814 \\
\hline 19 & rs73931385 & C & A & 0.0697 \\
\hline 19 & rs142357867 & $\mathrm{T}$ & G & 0.0348 \\
\hline 19 & rs73931386 & A & G & 0.0697 \\
\hline 19 & rs9630870 & A & G & 0.0581 \\
\hline 19 & rs6508953 & A & G & 0.0814 \\
\hline 19 & rs150311873 & $\mathrm{T}$ & C & 0.0465 \\
\hline 19 & rs7252852 & $\mathrm{T}$ & C & 0.3372 \\
\hline 19 & rs66657317 & G & GT & 0.0814 \\
\hline 19 & rs3852871 & C & A & 0.0697 \\
\hline 19 & rs201010762 & G & GA & 0.0697 \\
\hline 19 & rs17726493 & $\mathrm{T}$ & C & 0.0814 \\
\hline 19 & rs55790533 & A & G & 0.0814 \\
\hline 19 & rs113921300 & C & $\mathrm{T}$ & 0.0465 \\
\hline 19 & rs34724660 & A & G & 0.0697 \\
\hline 19 & rs3892666 & C & G & 0.1628 \\
\hline 19 & rs78367667 & G & A & 0.0697 \\
\hline 19 & rs73034462 & A & G & 0.3023 \\
\hline 19 & rs73034465 & G & A & 0.3023 \\
\hline 19 & rs7252501 & $\mathrm{T}$ & $\mathrm{C}$ & 0.1628 \\
\hline 19 & rs4358050 & A & G & 0.1628 \\
\hline 19 & rs4359558 & A & G & 0.3023 \\
\hline 19 & rs112531545 & G & C & 0.1628 \\
\hline 19 & rs4803404 & A & C & 0.1628 \\
\hline 19 & rs4468739 & C & $\mathrm{T}$ & 0.3023 \\
\hline 19 & rs4001944 & A & C & 0.3023 \\
\hline 19 & rs55779134 & GT & G & 0.3023 \\
\hline 19 & rs4001941 & G & A & 0.4767 \\
\hline 19 & rs34418474 & $\mathrm{T}$ & G & 0.0697 \\
\hline 19 & rs 12459860 & C & $\mathrm{T}$ & 0.0697 \\
\hline 19 & rs57274441 & G & $\mathrm{T}$ & 0.3023 \\
\hline 19 & rs4609955 & C & $\mathrm{T}$ & 0.3023 \\
\hline 19 & rs 12150973 & C & $\mathrm{T}$ & 0.3023 \\
\hline 19 & rs7255901 & C & $\mathrm{T}$ & 0.3023 \\
\hline 19 & rs 12459233 & G & C & 0.0697 \\
\hline 19 & rs35781447 & A & G & 0.0697 \\
\hline 19 & rs 12985721 & G & A & 0.0697 \\
\hline 19 & rs 28687008 & $\mathrm{~T}$ & C & 0.1512 \\
\hline 19 & rs145709497 & C & $\mathrm{T}$ & 0.0930 \\
\hline 19 & rs34151237 & G & $\mathrm{T}$ & 0.4186 \\
\hline 19 & rs34013487 & G & C & 0.2093 \\
\hline 19 & rs8107329 & $\mathrm{T}$ & C & 0.3023 \\
\hline 19 & rs74723889 & $\mathrm{T}$ & C & 0.0930 \\
\hline 19 & rs8100958 & C & $\mathrm{T}$ & 0.314 \\
\hline 19 & rs4124633 & C & $\mathrm{T}$ & 0.186 \\
\hline 19 & rs7245500 & C & A & 0.3837 \\
\hline 19 & rs11667592 & $\mathrm{C}$ & $\mathrm{T}$ & 0.0814 \\
\hline 19 & rs8109818 & G & A & 0.3837 \\
\hline 19 & rs73557157 & A & C & 0.2442 \\
\hline 19 & rs61586981 & G & A & 0.2442 \\
\hline 19 & rs60618302 & $\mathrm{T}$ & A & 0.2558 \\
\hline 19 & rs111588961 & $\mathrm{T}$ & C & 0.2442 \\
\hline 19 & rs73933714 & $\mathrm{T}$ & G & 0.4651 \\
\hline 19 & rs35950631 & $\mathrm{C}$ & A & 0.3953 \\
\hline 19 & rs16974790 & A & G & 0.3953 \\
\hline 19 & rs73933721 & A & G & 0.3953 \\
\hline 19 & rs16974794 & G & A & 0.3953 \\
\hline 19 & rs73559241 & G & A & 0.3953 \\
\hline
\end{tabular}

Table 1-A1 continues in the next column $\rightarrow$
Table 1-A1 (Continues...): Minor allele frequencies for 1846 polymorphisms in 43 black South Africans.

\begin{tabular}{|c|c|c|c|c|}
\hline Chromosome & Polymorphism & Minor allele & Major allele & $\begin{array}{l}\text { Minor allele } \\
\text { frequency }\end{array}$ \\
\hline 19 & rs8100458 & $\mathrm{C}$ & $\mathrm{T}$ & 0.186 \\
\hline 19 & rs59243457 & T & C & 0.3256 \\
\hline 19 & rs1872125 & C & $T$ & 0.3256 \\
\hline 19 & rs8101756 & C & $\mathrm{T}$ & 0.3256 \\
\hline 19 & rs8104022 & A & C & 0.3256 \\
\hline 19 & rs10409285 & $\mathrm{T}$ & C & 0.3256 \\
\hline 19 & rs62109048 & C & $\mathrm{T}$ & 0.3256 \\
\hline 19 & rs7250601 & C & A & 0.3256 \\
\hline 19 & rs2873264 & $\mathrm{T}$ & C & 0.1628 \\
\hline 19 & rs11672911 & A & G & 0.1628 \\
\hline 19 & rs2279341 & $\mathrm{C}$ & G & 0.0814 \\
\hline 19 & rs4803418 & G & C & 0.1163 \\
\hline 19 & rs12985017 & $\mathrm{C}$ & $\mathrm{T}$ & 0.0814 \\
\hline 19 & rs12985269 & C & $\mathrm{T}$ & 0.0814 \\
\hline 19 & rs4803419 & $\mathrm{T}$ & C & 0.0930 \\
\hline 19 & rs3745274 & $\mathrm{T}$ & G & 0.2907 \\
\hline 19 & rs2279345 & $\mathrm{T}$ & C & 0.1512 \\
\hline 19 & rs6508965 & $\mathrm{T}$ & C & 0.1512 \\
\hline 19 & rs6508966 & G & C & 0.1512 \\
\hline 19 & rs28399499 & $C$ & $\mathrm{~T}$ & 0.1163 \\
\hline 19 & rs8192719 & $\mathrm{T}$ & C & 0.2907 \\
\hline 19 & rs36118214 & A & G & 0.3256 \\
\hline 19 & rs11671243 & A & C & 0.1512 \\
\hline 19 & rs7260329 & A & G & 0.1047 \\
\hline 19 & rs7246465 & $\mathrm{T}$ & C & 0.1977 \\
\hline 19 & rs707265 & A & G & 0.1512 \\
\hline 19 & rs200843564_t3 & C & A & 0.3023 \\
\hline 19 & rs1042389 & C & $\mathrm{T}$ & 0.3023 \\
\hline 19 & rs56777936 & G & C & 0.0814 \\
\hline 19 & rs36002231 & C & $\mathrm{T}$ & 0.2093 \\
\hline 19 & rs34299754 & G & $\mathrm{T}$ & 0.2093 \\
\hline 19 & rs1552220 & A & G & 0.4302 \\
\hline 19 & rs1552221 & C & $\mathrm{T}$ & 0.4302 \\
\hline 19 & rs1552222 & A & $\mathrm{T}$ & 0.314 \\
\hline 19 & rs2113103 & A & G & 0.1047 \\
\hline 19 & rs60554840 & T & G & 0.1047 \\
\hline 19 & rs55869705 & A & G & 0.2326 \\
\hline 19 & rs10401226 & G & A & 0.2907 \\
\hline 19 & rs11666982 & T & G & 0.1512 \\
\hline 19 & rs112677106 & C & $\mathrm{T}$ & 0.2326 \\
\hline 19 & rs11670865 & $\mathrm{T}$ & G & 0.2907 \\
\hline 19 & rs7249735 & C & A & 0.2907 \\
\hline 19 & rs3745275 & A & G & 0.2907 \\
\hline 19 & rs7254767 & G & C & 0.3023 \\
\hline 19 & rs34855348 & A & G & 0.1047 \\
\hline 19 & rs73561518 & G & A & 0.1163 \\
\hline 19 & rs17799912 & T & C & 0.1047 \\
\hline 19 & rs113129391 & $\mathrm{T}$ & G & 0.1163 \\
\hline 19 & rs16974869 & C & $\mathrm{T}$ & 0.2326 \\
\hline 19 & rs73933730 & $\mathrm{T}$ & G & 0.2326 \\
\hline 19 & rs149490306 & A & C & 0.0697 \\
\hline 19 & rs16974893 & G & A & 0.2326 \\
\hline 19 & rs7250597 & $\mathrm{T}$ & C & 0.2326 \\
\hline 19 & rs12982859 & A & G & 0.1047 \\
\hline 19 & rs73561542 & $\mathrm{T}$ & G & 0.0581 \\
\hline 19 & rs11672352 & G & A & 0.1628 \\
\hline 19 & rs10407196 & G & C & 0.0814 \\
\hline 19 & rs13344213 & A & $C$ & 0.0814 \\
\hline 19 & rs113161508 & A & G & 0.0581 \\
\hline 19 & rs201853514 & TC & $\mathrm{T}$ & 0.2558 \\
\hline
\end{tabular}

Table 1-A1 continues on the next page $\rightarrow$ 
Table 1-A1 (Continues...): Minor allele frequencies for 1846 polymorphisms in 43 black South Africans.

\begin{tabular}{llccc}
\hline Chromosome & Polymorphism & Minor allele & Major allele & $\begin{array}{c}\text { Minor allele } \\
\text { frequency }\end{array}$ \\
\hline 19 & rs28502605 & C & T & 0.2558 \\
19 & rs7255149 & C & A & 0.4535 \\
19 & rs12459147 & G & A & 0.4651 \\
19 & rs34150638 & A & G & 0.4535 \\
19 & rs202055073 & AT & A & 0.0697 \\
19 & rs11672085 & T & C & 0.2791 \\
\hline
\end{tabular}

